\title{
A revision of Epithema (Gesneriaceae)
}

\author{
K. Bransgrove ${ }^{1,3}$ \& D.J. Middleton ${ }^{2}$ \\ ${ }^{1}$ Royal Botanic Garden Edinburgh, 20A Inverleith Row, \\ Edinburgh, EH3 5LR, Scotland, U.K. \\ ${ }^{2}$ Herbarium, Singapore Botanic Gardens, National Parks Board, \\ 1 Cluny Road, Singapore 259569 \\ david_middleton@nparks.gov.sg \\ ${ }^{3}$ Current address: Australian Tropical Herbarium, \\ James Cook University, Cairns Campus, Building E2, \\ P.O. Box 6811, Cairns, Queensland 4870, Australia
}

\begin{abstract}
The genus Epithema Blume (Gesneriaceae) is revised. A key is given, all species are described and several are illustrated. Twenty species are recognised and two new combinations are made, Epithema philippinum (Hilliard \& B.L.Burtt) Bransgrove and Epithema pusillum (C.B.Clarke) Bransgrove. Provisional IUCN conservation assessments are given for all species.
\end{abstract}

Keywords. Africa, Epithema, Gesneriaceae, Southeast Asia, taxonomic revision

\section{Introduction}

Epithema Blume is a genus of lithophytic herbs in central tropical Africa (Cameroon, Central African Republic, Democratic Republic of Congo, Equatorial Guinea, Guinea, Ivory Coast, Liberia, Sierra Leone and Uganda), South Asia (Nepal, India, Sri Lanka), Southeast Asia (southern China, Myanmar, Thailand, Vietnam, Cambodia, Laos, Taiwan), Malesia (Philippines, Malaysia, Indonesia) and the Solomon Islands.

The genus was first described by Blume (1826) with a single species, Epithema saxatile Blume. Aikinia R.Br. (in Wallich, 1832) was published shortly thereafter for a different species (Aikinia brunonis) but it was soon synonymised under Epithema by Decaisne (1834). Bentham (1835) and Spanoghe (1841) added further combinations or new taxa to Epithema but Don (1838) continued the use of Aikinia, placing Epithema in synonymy and moving Epithema carnosum to Aikinia. Later authors, however, correctly published treatments under the name Epithema. Major publications on the genus include two complete treatments of Epithema (Candolle, 1845; Clarke, 1883). Candolle (1845) included five species in his account and Clarke (1883) six species, of which five were from Asia and one from Africa. The account by Clarke (1883) also included eight new varieties and one new varietal combination. After 1883 there were occasional additional regional accounts and the descriptions of new taxa (Henriques, 1892; Chevalier, 1912; Merrill, 1916; Ohwi, 1943; Burtt, 1958; Kiew, 1985; Ying, 1992; Li \& Kao, 1998; Wang et al., 1998) and a set of brief but valid publications of nine new taxa and combinations by Hilliard \& Burtt (1997) in anticipation of a revision of the genus which, sadly, never materialised. 
The classification of the Gesneriaceae into subfamilies, tribes etc. has been rather unsettled (Weber et al., 2013) with the result that Epithema has been placed in a number of different subfamilies, tribes and series. Burtt \& Wiehler (1995) and Weber et al. (2013) both placed Epithema in the tribe Epithemateae (Meisn.) C.B.Clarke of the subfamily Didymocarpoideae (called Cyrtandroideae by Burtt \& Wiehler (1995)). Weber et al. (2013) further placed it in its own subtribe, Epitheminae. Mayer et al. (2003), in a molecular phylogenetic study of the group, found that the Epithematoid Gesneriaceae are sister to the Didymocarpoid Gesneriaceae and that Epithema is monophyletic and sister to a clade with a single species each of Loxonia Jack and Stauranthera Benth. The study only included four species of Epithema (E. tenue, E. saxatile, E. membranaceum and E. taiwanense [= E. ceylanicum]) and, therefore, provides little information about the interrelatedness of the species within the genus.

In flower, Epithema is not easily confused with other genera although some specimens have been previously mistaken for Monophyllaea R.Br. due to a superficial resemblance to some species. The two genera can be separated by inflorescence arrangement, sepal shape and aestivation, placentation, the number of locules in the ovary, seed shape and hair type.

The species of Epithema are not readily distinguished from each other. Clarke (1883) suggested that the then known five Asian species could possibly be recognised as only one. Many are similar and there are few good characters to separate the species as both vegetative and reproductive characters can be variable. In particular, the species from mainland Asia are very similar and difficult to separate.

The gender of Epithema is neuter. Clarke (1883), however, described several new varieties with feminine endings. These have been corrected to neuter in the revision below.

\section{Morphological Characters}

Epithema is primarily lithophytic. In eastern and southeastern Asia it is found on limestone and, to a lesser extent, on granite and sandstone. It appears to be restricted to limestone in Borneo. In Africa, India and non-peninsular Myanmar it is found on volcanic rocks and soils. It requires shaded conditions with high humidity and is frequently found near or in cave entrances, on rocks on the forest floor, or on rocks beside, or in, shallow streams and rivers. Information from specimen labels indicates Epithema species will occasionally grow on fallen trees and is often collected from road sides and road side embankments.

All species of Epithema are small herbs. Epithema, like other didymocarpoid Gesneriaceae, is anisocotyledonous with the cotyledons withering early. The lowermost true leaf, however, is solitary and looks rather like the macrocotyledon found in other genera such as Monophyllaea (Weber, 2004).

The majority of species are caulescent but a few species have a very short stem with leaves arising more or less at ground level, therefore appearing acaulescent (Epithema dolichopodum, E. longipetiolatum, E. philippinum, E. rennellense, E. strigosum and some specimens of E. benthamii, E. ceylanicum and E. tenue). One 
caulescent species, however, often exhibits a more sprawling rather than an upright habit (E. sarawakense) and one is unifoliate in most of its distribution (E. horsfieldii).

The hairs on all plant parts are simple, unbranched, straight or hooked, have 3-5 cells and are from $0.02 \mathrm{~mm}$ to (rarely) $1.5 \mathrm{~mm}$ long. Hooked hairs are usually shorter than straight hairs. The type of hairs (pubescent, strigose, setose, hispid or villous), density (glabrous to dense), colour (hyaline to brown) and length are quite variable. The combination of these characters is useful in some species but in others it is often too variable to be of any taxonomic use. It is, therefore, included in the descriptions only where it is taxonomically useful. Where 'minute hooked pubescence' is included in descriptions it denotes hooked hairs of a maximum of $0.1 \mathrm{~mm}$ long.

The leaves of Epithema are thinly to thickly membranous and are usually narrowly to broadly ovate, more rarely oblong to linear. The apex of the leaves is rounded to acute, the margin entire to serrate, dentate or crenate (or variations thereof) and the bases cordate to truncate to occasionally attenuate. The upper surface of the leaves is green to dark or black-green and the lower surface is usually lighter in colour than the upper surface and may dry whitish green to grey-green. Specimens of Epithema saxatile and E. ceylanicum, and possibly others, show some green and silver variegation on the upper surface of the leaves. The variegation has only been seen in a small number of specimens from Thailand and Peninsular Malaysia. The indumentum of the upper surface is variable in composition and the hairs are up to $1.5 \mathrm{~mm}$ long. The indumentum of the lower surface is similar to that of the upper surface but the hairs are usually sparser, shorter and finer. The indumentum of the leaves is occasionally taxonomically useful and is only included in the descriptions in those cases.

The leaf venation is cladodromous with the secondary veins mostly strongly ascending. There are often weaker intersecondaries and the tertiary venation is mostly fairly obscure but, when visible, laxly alternate percurrent. The number and arrangement of secondary veins is variable, between 4 and 15 and alternate to opposite (or both within one leaf), and not taxonomically important.

Leaf arrangement is variable. Caulescent species can have opposite or solitary leaves that in turn may be petiolate or sessile. A combination of all leaf arrangements may be found on one plant or within a population. Most acaulescent species have unpaired leaves. When present, solitary upper leaves are nearly always petiolate and opposite one or more peduncles.

Weber (2004) described the inflorescence of Epithema as "reduced to a single bract/inflorescence unit" which is derived from a "more elaborate thyrse, with several bract/inflorescence units in alternate position". The appearance is of a small, multiflowered, scorpioid cyme. The flowers are arranged into rows, two to three across. The indumentum of the peduncle is glabrous to hispid with hairs to $1.25 \mathrm{~mm}$ long. The flowers are collectively subtended by one bract which is green and frequently cucullate, partially or entirely enclosing the inflorescence, but can be very small. The uppermost margin of the bract is usually dentate but can be entire to dentate. If the margin of the bract is lobed, the margin of the lobes may be entire, dentate or crenate. The indumentum of the lower surface of the bract is of a short, hooked pubescence and/or strigose to setose, straight and/or hooked hairs to $1 \mathrm{~mm}$ long. The indumentum 
of the upper surface is usually of one hair type only, towards the margin and to 0.6 $\mathrm{mm}$ long.

The flowers mature sequentially from the base of the inflorescence towards the apex. The apex is always curved over to more or less horizontal and is often covered by the bract which subtends the entire inflorescence. The cells at the base of the inflorescence continue to grow so that the inflorescence changes position as flowers mature and the open flower is presented between the horizontal and around $45^{\circ}$. The number and size of the flowers in the inflorescence and the size and shape of the bract varies between and sometimes within species but the size of the bract is a useful character for the recognition of many species.

The pedicel is short, mostly up to only $5 \mathrm{~mm}$ long, and can elongate considerably at maturity but only rarely to as long as $7.5 \mathrm{~mm}$. The calyx consists of five sepals that are fused from the base of the calyx to half or two-thirds the length of the sepals. It is actinomorphic, persistent and expands as it matures, providing a receptacle for the operculate capsule that sits in the centre of the calyx. The young calyx is generally oblong to obovate and becomes more campanulate at maturity. The lobes are linear to triangular, often becoming broader and acuminate in maturity. The shape of the calyx does not vary significantly between species.

The indumentum of the pedicel and calyx is similar to that on the bract and in many species is the same on both structures. Hairs on the pedicel are to $1 \mathrm{~mm}$ long, to $1.5 \mathrm{~mm}$ long on the outside of the calyx and to $0.5 \mathrm{~mm}$ long on the inside of the calyx. If present, the hairs on the inside of the calyx are usually strigose and found only on the lobes of the calyx. The combination of type and densities of the hair on the outside of the calyx is much more variable than on the inside of the calyx. Despite the variability it can be a useful character for species differentiation (e.g. E. tenerum and E. steenisii).

The corolla consists of a tube and five free corolla lobes in two lips. The tube accounts for approximately two thirds to three quarters of the length of the corolla. The smaller upper lip is of two lobes and the lower of three lobes. The upper lip is sometimes much smaller than the lower. The colour of the corolla is variable. Specimen labels indicate that the flowers may be white or pink, but usually the tube is white or of unstated colour and the lobes are blue to violet. The flowers of many species have irregular darker (purple) marks at the base of the lobes or in the upper throat.

In most species there are villous hairs on the inside of the corolla tube and there may be a minute pubescence on the outside of the lobes. The villous hairs are often in a dense band around the perimeter of the corolla tube and are usually placed somewhere between half way and two-thirds of the way up the tube and below the stamens. The tube is rarely glabrous or pubescent externally with short hairs inside. The presence and type of hairs inside the corolla is a useful character for separating some species of Epithema, while the hairs on the outside of the corolla lobes are not. Hairs inside the corolla can be difficult to see in rehydrated material. The hairs on the outside of the corolla can be difficult to observe and are not necessarily persistent. They are not included in the species descriptions.

The androecium consists of two fertile stamens and two staminodes. The staminodes are a filament with an arrow- or serpent's-head-shaped end. They are often 
slightly shorter than the filaments of the fertile stamens. The stamens and staminodes are glabrous. The third, medial, staminode present in many Gesneriaceae is not seen in Epithema. The anthers are apically coherent, the thecae are divaricate and apically confluent. The stamens and staminodes are inserted on the top of a more or less square flap of androecial tissue which is in turn inserted on the wall of the corolla. The flap of tissue is up to $2.5 \mathrm{~mm}$ wide and high. The stamens are inserted on either end of the flap and the staminodes to the inside of each of them. The upper edge of the flap of tissue may be rounded leaving the staminodes at a slightly higher plane than the fertile stamens, up to $0.5 \mathrm{~mm}$ higher. The head of the staminode is frequently adjacent to, but not joined to, the back of the anther or the very top of the stamen filament. The style grows through the space between the flap and the two coherent anthers. The androecial tissue is normally inserted about half way to two-thirds of the way up the wall of the corolla tube, but can be placed higher in the tube. Irrespective of the position on the corolla tube, the placement of the stamens in relation to the staminodes does not change.

The nectary is comprised of one, two or three discrete lobes. In many species there are two lobes, one on each side of the ovary. Sometimes the nectary lobe(s) completely enclose the ovary, while in others the nectary is apparently absent. The composition of the nectary is variable within and between species and is not a good taxonomic character.

The ovary is superior, spherical to cylindrical and has one locule. The shape is variable and is not taxonomically useful. The placentation is parietal with two 'stalked' placentae, one on each side of the ovary. The placentophores are fused in their lower half to the ovary wall while the upper half supporting the placenta is free. This allows the formation of a dehiscent operculum in fruit. The ovules are produced from, and connected to, the placenta at the head of the placentophore by individual funicles. The upper portion of the ovary is usually pubescent and the lower portion is glabrous. Like the remainder of the plant, the indumentum consists of straight and/or hooked hairs. The hairs are not always entirely persistent, but density, length and shape of the hairs on the ovary are taxonomically useful. Unfortunately, the hairs are short (usually 0.1-0.3 mm long) and not easily observed and, therefore, are of limited use for field identification. The stigma is bilobed and the surface is covered in minute protrusions or papillae of c. $0.1 \mathrm{~mm}$ long. The style is slender and is inserted into the ovary in the centre of the upper margin of the ovary. The style may be glabrous to densely pubescent, the hairs being from $0.04-0.3 \mathrm{~mm}$ long and placed at the base of the style. Rarely are they up to mid-way on the style.

The fruit of Epithema is a circumscissile or operculate capsule which is oblong, obovate or sub-spherical in shape. The indumentum on the operculum is the same as on the ovary although it may be sparser. After fertilisation the style withers and breaks off leaving a small portion $0.3-2.3 \mathrm{~mm}$ long. This remnant is persistent and seen on the mature operculum. As the fruit matures, movement of the inflorescence brings the fruit into a vertical position, the calyx lobes grow and open widely outwards, leaving a basin-like structure in which sits the mature fruit. At maturity, the operculum is lost, leaving the seeds open to the elements. The hair on the operculum is a useful 
taxonomic character, while fruit shape, size and length of the style remnant are not.

A large number of seeds is produced per capsule. The seeds are narrowly to broadly ovoid, rarely sub-cylindrical to slightly sigmoid in shape, with acute and/or constricted ends. They are not appendaged and they separate entirely from the funicle by which they connect to the placenta. They vary from light to dark brown and the ends are darker than the main body of the seed. Colour can vary between capsules of the same inflorescence and is certainly associated with stages of maturity, becoming darker with age. Some variation in colour might also be an artefact of drying.

The surface of the seed coat of all species of Epithema is arranged into more or less parallel lines that, when viewed under magnification, are darker than the remainder of the seed coat. The lines are straight to spiralled in the direction of the long axis of the seed and are between 0.02 and $0.05 \mathrm{~mm}$ apart. These longitudinal lines may fork and cross-walls are often present between the longitudinal lines. The density of the cross-walls and the forking does vary, giving some seeds a sub-reticulate look. Older descriptions place importance on the angle of the longitudinal lines, but angle of the pattern varies considerably even between seeds from one capsule or different capsules of one inflorescence. Scanning electron microscope (SEM) photographs of the seed of a small number of species of Epithema indicate that the surface of the seed is not taxonomically useful (Michael Moeller and Frieda Christie, pers. comm.). Further examination of the seed by SEM was not conducted in this revision. In only a few species is the length of the seed useful.

Splash cup dispersal is the primary means of seed dispersal in Epithema. The splash cups are formed from the persistent calyx and seeds are dispersed directly from the capsule, usually by raindrops. Splash dispersal is usually only effective over short distances but it is also possible that seed may be transported longer distances on the fur or the feet of animals, or in streams.

\section{Materials and Methods}

Approximately 1400 specimens from the following herbaria have been examined: A, AAU, ABD, BK, BKF, BM, BO, C, CAL, CALI, CMU, COI, E, G, GH, K, KEP, KLU, KYO, L, MSC, MO, NY, P, PDA, PNH, PSU, QBG, S, SAR, SING, TI, UPM, US and WAG (Thiers, continuously updated). These specimens were collected between the 1820s and 2014 from Cameroon, Central African Republic, Democratic Republic of Congo, Equatorial Guinea, Guinea, Ivory Coast, Liberia, Sierra Leone and Uganda, Sri Lanka, India, Nepal, China, Taiwan, Myanmar, Thailand, Laos, Cambodia, Vietnam, Malaysia, Singapore, Philippines, Indonesia, Timor Leste and the Solomon Islands. All specimens cited have been seen unless otherwise indicated. All measurements for vegetative characters, peduncles, bracts, pedicels and calyces are from dried specimen material. Measurements of other reproductive structures are taken from rehydrated material. Observations on herbarium material have been augmented by observations in the field (in Malaysia and Thailand) by both authors.

There are relatively few collections from areas such as eastern Thailand, Laos, 
Cambodia, Vietnam, Sulawesi, Maluku, The Sunda Islands, Timor and Timor Leste, New Guinea and the many small islands of south-eastern Asia. In some of these areas Epithema species may genuinely be absent or scarce due to the lack of ideal habitats (e.g. absence of limestone) but collecting densities are known to be low in several of these areas (Johns, 1995; Middleton, 2003; Newman et al., 2007). Peninsular Malaysia and other areas of Thailand are relatively well collected.

Provisional conservation assessments are made using the methodology proposed by IUCN (2001).

The species are presented in alphabetical order as little data is available on relationships between the species. The dots on the maps represent the distributions of the specimens cited in this revision.

The flowering and fruiting times given are based on the data from herbarium specimens only and do not preclude flowering and fruiting at other times of year.

\section{Constraints}

Flowers in an Epithema inflorescence mature sequentially within the inflorescence with only one or few flowers fully expanded at any one time. In addition, the corolla is not persistent. It is, therefore, common for each specimen or sheet of specimens to contain no corolla material. This means that a thorough evaluation of infraspecific variability in corolla, stigma, style and stamen characters was limited by the availability of flowering material. When only immature flower buds were present they were dissected to gather character states for corolla and ovary pubescence. It is acknowledged that character states could change during the maturation process but we still found these bud characters useful.

For a number of species there are very few available specimens. This limits our understanding of the variability and distribution of the species.

Plant description, expedition date and/or geography data was limited or absent from many labels of the older specimens. For species with only small numbers of older specimens, distributions below country level are unattainable and transient character states such as corolla colour remain unknown.

\section{Epithema Blume}

Bijdr. 737 (1826); C.B.Clarke in A.DC. \& C.DC., Monogr. Phan. 5(1): 176 (1883); C.B.Clarke in Hook.f., Fl. Brit. Ind. 4: 369 (1884); King \& Gamble, J. Asiat. Soc. Bengal, Pt. 2, Nat. Hist. 74(2): 783 (1909); Ridl., Fl. Malay Penins. 2: 539 (1923); Gamble, Fl. Madras 2: 991 (1924); Pellegr., Fl. Indo-Chine 4: 558 (1930); Kanjilal et al., Fl. Assam 3: 399 (1939); Barnett, Fl. Siam. 3(3): 205 (1962); Backer \& Bakh.f., Fl. Java 2: 527 (1965); W.L.Theob. \& Grupe in Dassan. \& Fosberg, Revis. Handb. Fl. Ceylon 3: 102 (1981); Pham-Hoàng Hô, Cayco Vietnam ed. 3, 3(1): 24 (1993); W.T.Wang et al., Fl. China 18: 400 (1998); B.L.Burtt, Thai For. Bull. (Bot.) 29: 93 
(2001); Hilliard in Grierson \& D.G.Long, Fl. Bhutan 2(3): 1328 (2001); A.Weber in Kubitzki, Fam. Gen. Vasc. Pl. 7: 129 (2004). - TYPE: Epithema saxatile Blume. Aikinia R.Br. in Wall., Pl. Asiat. Rar. 3: 65 (1832). - TYPE: Aikinia brunonis Wall. (= Epithema brunonis (Wall.) Decne.).

Carpocalymna Zipp., Alg. Konst- Lett.-Bode 1: 297 (1829), nom. nud.

Herb, caulescent or acaulescent, occasionally only one leaf developing. Leaves: lowest leaf petiolate and solitary, upper leaves petiolate or sessile, opposite or alternate; blades thinly to thickly membranous, usually ovate to cordate, more rarely elliptic to orbicular, if asymmetrical then the wider side up to 1.75 times as wide as the narrower side, apex rounded to acute, base mostly cordate or sub-auriculate to obtuse, inserted evenly or unevenly on petiole, margin entire to crenate, serrate or (bi-)dentate, venation pinnate, upper surface pale to black-green, sometimes variegated, lower surface light to midor olive-green or purplish. Inflorescences 1-15(-many) per plant; peduncles usually originating from the leaf axils, occasionally from the petiole and/or the midrib of the blade; singular bract subtending each inflorescence, cucullate and enclosing the entire inflorescence to reduced, margin entire to dentate. Calyx cylindrical to campanulate, consisting of a tube and 5 lobes, with an embedded gland towards the apex of each lobe. Corolla tube usually white, lobes pale pink to blue or purple, commonly with darker markings on either lip; tube cylindrical to narrowly fluted, occasionally slightly constricted at the apex; lobes with margins entire to fimbriate. Stamens and staminodes inserted on top of flap of androecial tissue which is inserted in corolla tube; fertile stamens 2; anthers coherent at thecae tips or along entire thecae; staminodes 2, third staminode absent. Nectary apparently absent or one- to three-lobed, margin entire to undulate. Ovary cylindrical to spherical, glabrous to densely pubescent, unilocular, placentation parietal; style short; stigma bi-lobed, papillate, glabrous. Fruit (sub-) cylindrical to (sub-)spherical; operculum circumscissile or irregularly dehiscing at maturity, indumentum as ovary; surrounded by persistent calyx. Seed usually narrowly ovoid to broadly ovoid, ends acute and/or constricted, light to dark brown with darker ends, pattern straight to spiralled, walls of pattern usually splitting and merging and with cross-walls, occasionally walls of pattern appear thickened and rigid.

Twenty species from central tropical Africa, India, Sri Lanka, Nepal, southern China and through Southeast Asia and Malesia to the Solomon Islands.

\section{Key to Epithema species}

1a. First leaf arising more or less at ground level or from a very short stem ...... 2

1b. First leaf arising above a definite stem ............................... 10

2a. Indumentum on ovary/operculum straight or primarily straight ............ 3

2b. Indumentum on ovary/operculum hooked or primarily hooked, operculum rarely glabrous 
3a. Ovary/operculum indumentum mostly villous with hairs to $1.5 \mathrm{~mm}$ long; corolla $>9 \mathrm{~mm}$ long; Africa ............................. 20. E. tenue p.p.

3b. Ovary/operculum indumentum generally not villous, $<1 \mathrm{~mm}$ long (usually

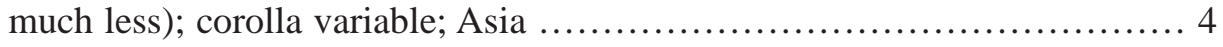

4a. Hairs on ovary/operculum both straight and hooked; Solomon Islands

14. E. rennellense p.p.

4b. Hairs on ovary/ operculum straight only; not Solomon Islands ............ 5

5a. Leaf margin entire or only minutely toothed, more rarely with larger and irregular teeth; Eastern Indonesia ................... 7. E. longipetiolatum p.p.

5b. Leaf margin distinctly toothed; widespread in Malesia .................... 6

6a. Ovary/operculum hairs to $0.6(-1) \mathrm{mm}$ long; upper leaf surface with only strigose to villous hairs with at least some hairs $>1 \mathrm{~mm}$ long; Philippines and eastern Malesia ........................................ 1. E. benthamii p.p.

6b. Ovary/operculum hairs to $0.3 \mathrm{~mm}$ long; upper leaf surface sometimes with minute hair covering as well as longer hairs but all hairs $<1 \mathrm{~mm}$ long;

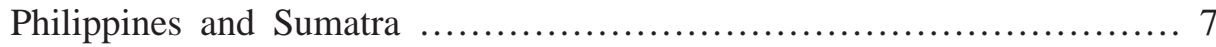

7a. Leaves strongly crenate or with large, wide teeth with rounded apices; bracts > 10 mm long; Sumatra 18. E. strigosum

7b. Leaves dentate/serrate, teeth narrow with pointed apices; bracts < $10 \mathrm{~mm}$ long; Philippines 12. E. philippinum

8a. Hairs on ovary/operculum hooked or hooked and straight; corolla 10.4-15 mm long; Solomon Islands ........................... 15. E. rennellense p.p.

8b. Hairs on ovary/operculum only hooked or operculum rarely glabrous; corolla 5-8.5 mm long; not Solomon Islands

9a. Inflorescences 1-5 per plant, usually large and not in fascicles; peduncles (2-)9-27 cm long; bracts 10-25 × 6-14 mm; operculum rarely glabrous; Sabah 4. E. dolichopodum

9b. Inflorescences 1 to many, small, often different lengths, in fascicles; peduncles up to 10(-17) cm long; bracts 3-16(-20) × 3-10 mm; operculum with hooked hairs; continental Asia, Taiwan, Philippines

3. E. ceylanicum (rare acaulescent form)

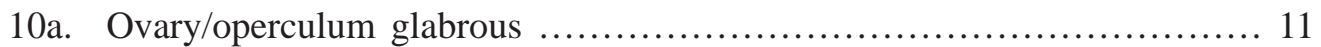

10b. Ovary/operculum pubescent or at least with few hairs .................. 14

11a. Plants with only 1 leaf, occasionally with 2 leaves, these never opposite; inflorescences arising from leaf blade midrib or junction of petiole and midrib 5. E. horsfieldii p.p.

11b. Plants usually with more than 2 leaves, leaves alternate or opposite; inflorescences in the leaf axils or opposite a solitary leaf ............... 12 
12a. Upper leaves sessile 9. E. madulidii p.p.

12b. Upper leaves petiolate 13

13a. Leaves all or primarily alternate; $1-5$ peduncles arising in sequence from base of petiole; bracts large (10-32 × 6-12 mm) and usually enclosing inflorescence; plants with strigose or setose hairs, inner calyx glabrous or with sparse straight strigose hairs; Malesia ..............15. E. sarawakense p.p.

13b. Leaves opposite; peduncles arising from leaf axils; bracts small (4-8 $\times$ 4-9 $\mathrm{mm}$ ) and not enclosing inflorescence, plants glabrous to sparsely pubescent, inner calyx glabrous; southern India ....................... 13. E. pusillum

14a. Plants with only 1 leaf, occasionally with 2 leaves, these never opposite; inflorescences arising from leaf blade midrib or junction of petiole and midrib, ovary hair hooked

5. E. horsfieldii p.p.

14b. Plants usually with more than 2 leaves, upper leaves usually opposite; inflorescences from base of petioles or in the axils of upper petiolate or sessile leaves, rarely at base of midrib in the rare case leaves are alternate, 1-5 peduncles arising in sequence from base of petiole

15a. Upper leaves sessile ............................................. 16

15b. Upper leaves petiolate ............................................ 19

16a. Indumentum on ovary/operculum straight or primarily straight; Africa 20. E. tenue p.p.

16b. Indumentum on ovary/operculum hooked or primarily hooked; not Africa

17a. Corolla glabrous inside; Philippines 9. E. madulidii p.p.

17b. Corolla pubescent inside 18

18a. Bract enclosing inflorescence, 13-35 × 8-12 mm 16. E. saxatile p.p.

18b. Bract not enclosing inflorescence, 4-16 $\times 4-9 \mathrm{~mm}$ 3. E. ceylanicum p.p.

19a. Indumentum on ovary/operculum straight or primarily straight 20

19b. Indumentum on ovary/operculum hooked or primarily hooked 24

20a. Indumentum on ovary to $1.5 \mathrm{~mm}$ long; Africa 20. E. tenue p.p.

20b. Indumentum on ovary $<1 \mathrm{~mm}$ long; Asia

21a. Bracts cucullate, (2-)5-23 × (3-)4-11 mm; Philippines, eastern Malesia ... 22 21b. Bracts not cucullate, 1-13 × 2-9 mm; continental Asia and Peninsular Malaysia 23 
22a. Leaves mostly not opposite, only occasionally so and then often of markedly different sizes; leaf margin entire or only minutely toothed, more rarely with larger and irregular teeth ........................ 7. E. longipetiolatum p.p.

22b. Upper leaves usually opposite, only rarely upper leaves solitary; leaf margin distinctly and usually regularly toothed 1. E. benthamii p.p.

23a. Bract small, rarely sub-cucullate or trullate, not enclosing inflorescence, 1-7 $\times$ 2-7 mm, margin often deeply lobed with 3 lobes; plants often with dense white hairs on most plant parts; corolla 4.2-5.4 mm long; Peninsular Malaysia 11. E. parvibracteatum p.p.

23b. Bract small or sub-cucullate, enclosing a small portion of the inflorescence, 1-13 × 3-9 mm, margin entire to dentate; plants sub-glabrous to pubescent; corolla 5.5-7.5 mm long; Myanmar, China, India, Laos, Nepal, Thailand

2. E. carnosum

24a. Corolla glabrous inside

6. E. involucratum

24b. Corolla with hair (villous) inside 25

25a. Leaves all or primarily alternate; 1-5 peduncles arising in sequence from base of petiole; bracts large (10-32 × 6-12 mm) and usually enclosing inflorescence; Malesia ...........................15. E. sarawakense p.p.

25b. Leaves primarily opposite; number and position of peduncles variable; bracts small to large and enclosing inflorescence or not; Continental Asia and Malesia 26

26a. Corolla $>10 \mathrm{~mm}$ long 8. E. longitubum

26b. Corolla $<10 \mathrm{~mm}$ long 27

27a. Peduncles arising along petiole, often in fascicles, often many and of very different lengths 3. E. ceylanicum p.p.

27b. Peduncles primarily arising from axils of leaves, usually singly or few and then of not remarkably different length 28

28a. Bract only rarely sub-cucullate or trullate but not enclosing the inforescence, $1-11 \times 2-12 \mathrm{~mm}$ 29

28b. Bract sub-cucullate to cucullate enclosing a portion or all of the inflorescence, (3-)6-23 × 3-12 mm 31

29a. Lower to mid-style pubescent; calyx without hispid white hairs; delicate growth form 10. E. membranaceum

29b. Style glabrous or with only a few hairs; calyx indumentum variable but with hispid white hairs; often with a robust growth form 30 
30a. Plants with dense white hairs on most plant parts; Peninsular Malaysia 11. E. parvibracteatum p.p.

30b. Plants not densely pubescent; not in Peninsular Malaysia 3. E. ceylanicum (rare form with few inflorescences and smaller bracts)

31a. Bract usually enclosing only a portion of the inflorescence 32

31b. Bract usually enclosing most or all of inflorescence; corolla $6.5-9.5 \mathrm{~mm}$ long

32a. Plant 5-14(-20) cm; leaves often broadly lanceolate to narrowly ovate; corolla 5.2-6 mm long; Sulawesi ................................. 19. E. tenerum

32b. Plant 4-40 cm; leaves usually cordate, ovate or elliptic; corolla 5-8.5 mm long; not in Sulawesi ................................... 3. E. ceylanicum p.p.

33a. Calyx with strigose hairs only outside, hairs $0.5-0.8 \mathrm{~mm}$ long; Aceh ......... 17. E. steenisii

33b. Calyx often glabrous, if hairs present these $<0.5 \mathrm{~mm}$ long; Myanmar, Thailand, Malaysia, Sumatra, Java, Borneo, Sulawesi ...... 16. E. saxatile p.p.

1. Epithema benthamii C.B.Clarke in A.DC. \& C.DC., Monogr. Phan. 5(1): 180 (1883). - TYPE: Philippines, Albay, 1841, Cuming, H. 1265 (lectotype K [K000438696], designated here; isolectotype BM, K [K000438697]). (Fig. 1, 2)

Epithema brunonis var. scabridum C.B.Clarke in A.DC. \& C.DC., Monogr. Phan. 5(1): 178 (1883). - TYPE: Indonesia, Sulawesi, Gorontalo, Riedel s.n. (holotype K).

Epithema calcicola Ohwi, Bot. Mag., Tokyo 57: 125 (1943). - TYPE: Indonesia, West Papua, Waren, 60 miles south of Manokwari, 26 March 1940, Kanehira, R. \& Hatusima, S. 13143 (lectotype FU, image seen, designated here; isolectotype BO).

Herb 5-30 cm high, caulescent, occasionally appearing acaulescent with leaves at near ground level, sparsely to densely strigose and/or setose with hairs to $0.8 \mathrm{~mm}$ long, the lower bract, pedicel and the outside of the calyx also minutely pubescent with hairs to $0.2 \mathrm{~mm}$ long; stem 1-4 mm wide with 2-3 nodes, internodes $0.5-6.5 \mathrm{~cm}$ long. Leaves strongly membranous, upper leaves petiolate and opposite, infrequently with a solitary upper leaf; petiole of lowest leaf $2-13.5 \mathrm{~cm}$ long, upper petioles $0.2-6 \mathrm{~cm}$ long; blade of lowest leaf 4.3-14.5 × 2.2-7.5 cm; blades of upper leaves $2-12 \times 1-6 \mathrm{~cm}$, all leaves linear, narrowly ovate, or almost oblong, occasionally ovate or rhomboid, usually symmetrical, apex acute to rounded, base sub-cordate, rounded to truncate, occasionally sub-auriculate, usually inserted evenly on the petiole, margin usually irregularly serrate or biserrate; upper surface mid-green to black-green, sparsely to densely weakly strigose to villous, without minute hairs interspersed, straight hairs to $1.5 \mathrm{~mm}$ long, hooked hairs $0.5 \mathrm{~mm}$ long; lower surface generally lighter in colour than 


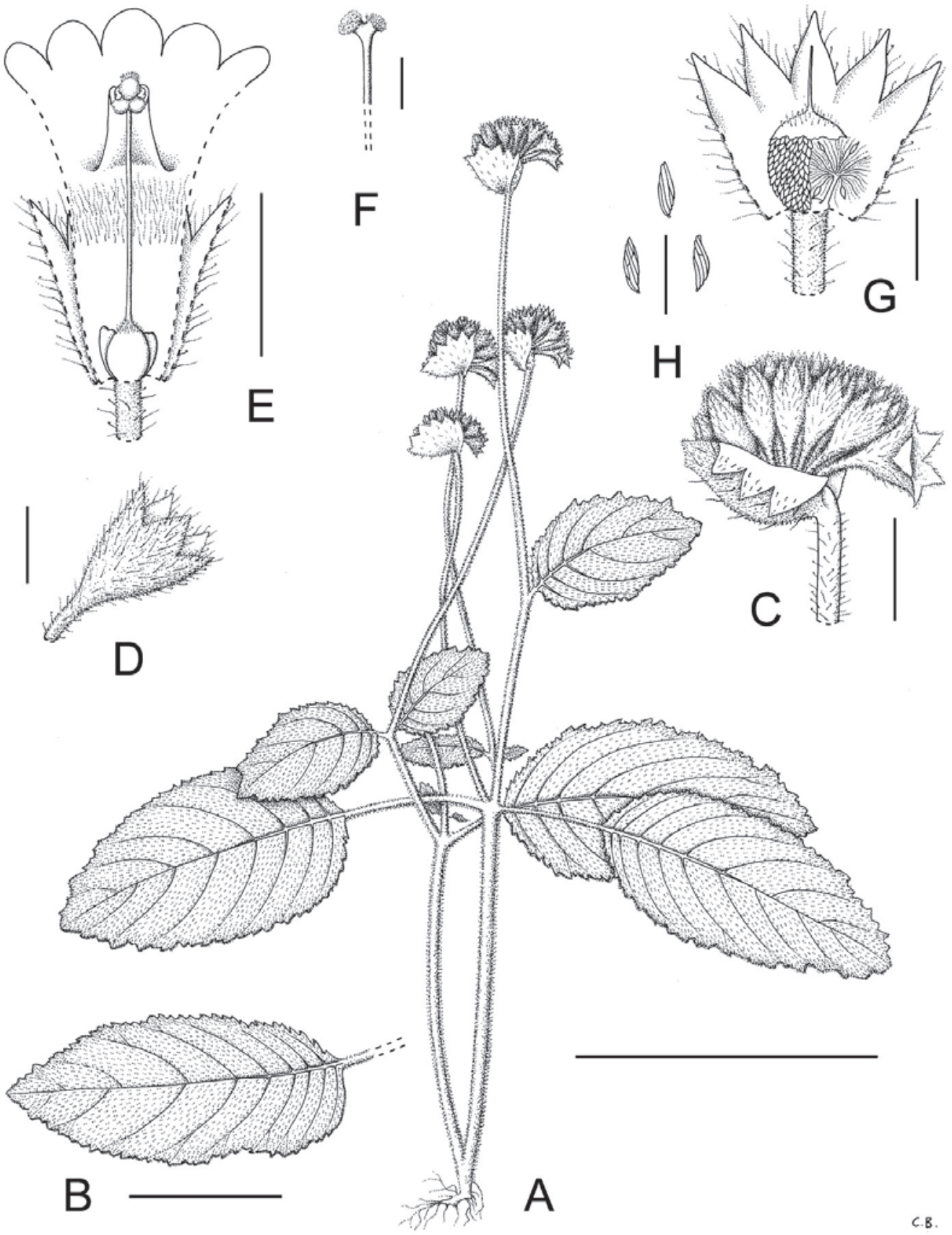

Fig. 1. Epithema benthamii C.B.Clarke. A. Habit. B. Leaf. C. Inflorescence. D. Calyx. E. Flower opened out. F. Stigma lateral view. G. Fruit showing seeds, placenta and operculum. $\mathbf{H}$. Seeds. Scale bars: $A=6 \mathrm{~cm}, B=3 \mathrm{~cm}, C=5 \mathrm{~mm}, \mathrm{D}=3 \mathrm{~mm}, \mathrm{E}=4 \mathrm{~mm}, \mathrm{~F}=1 \mathrm{~mm}, \mathrm{G}=2 \mathrm{~mm}$, $\mathrm{H}=0.5 \mathrm{~mm}$. Drawn by Claire Banks from Wenzel 437 (A, C-H) and Coode 5313 (B). 


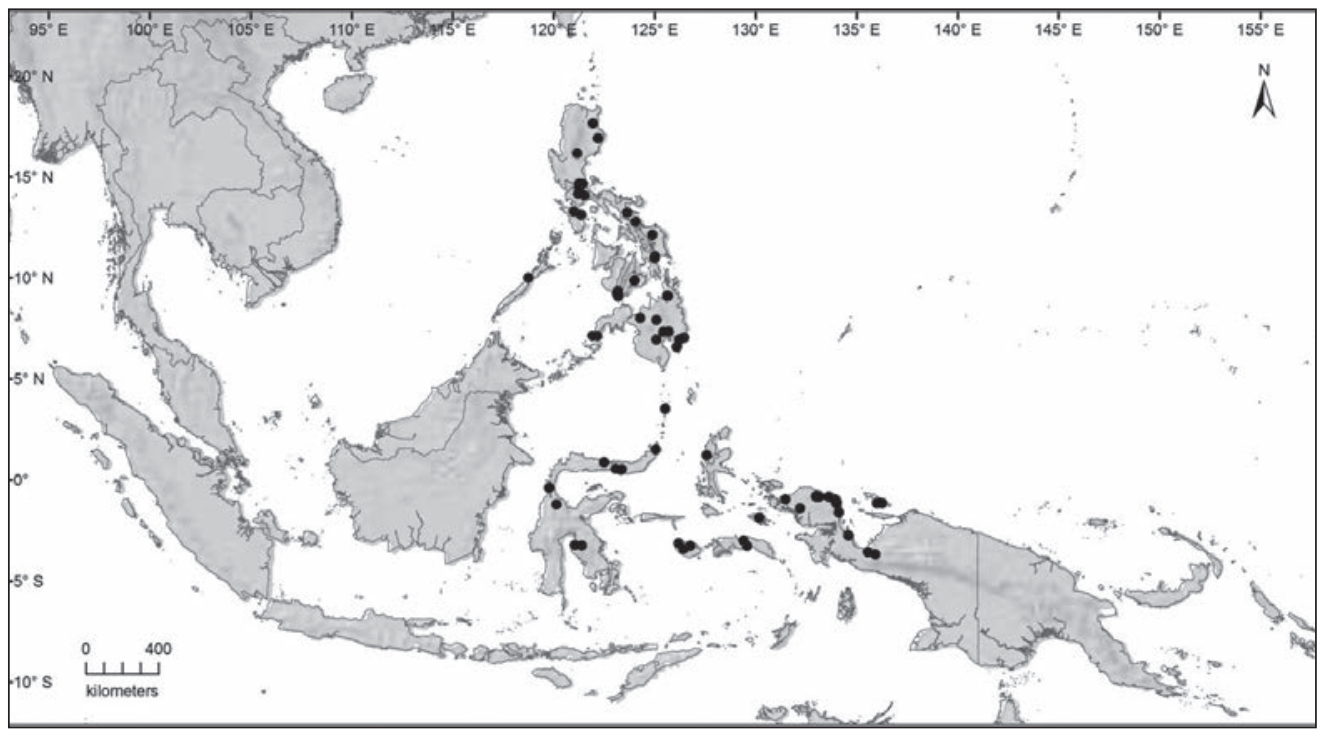

Fig. 2. Distribution of Epithema benthamii C.B.Clarke (•).

upper surface. Inflorescences 1-4(-6) per plant; peduncles 2.5-16 cm long, arising from leaf axils or, rarely, from the base of the uppermost petioles; bracts sub-cucullate or cucullate, enclosing a proportion of the inflorescence, occasionally reduced, (2-)7$23 \times(3-) 5-11 \mathrm{~mm}$, margin usually dentate with large teeth but entire if reduced, lower surface with long strigose hairs 0.6-1 mm long, upper surface glabrous to pubescent at upper margin with hairs to $0.5 \mathrm{~mm}$ long; pedicels $1.5-4 \mathrm{~mm}$ long. Calyx 2.4-6 mm long; tube 1.7-3 × 1.5-2.5 mm; lobes 1.7-3 × 0.8-1.5 mm; glabrous inside, rarely with few straight hairs to $0.1 \mathrm{~mm}$ long in lobes. Corolla often white, but also blue to purple, 5-10 mm long; tube (4-)5-6(-8) × 1.3-2.5 mm; lobes 1-2 mm long. Stamens 1.4-1.5 mm long; filaments $1 \mathrm{~mm}$ long; anthers $0.4-0.5 \mathrm{~mm}$ long; staminodes $0.8-0.9$ $\mathrm{mm}$ long. Nectary apparently absent or of two lobes, $0.8-1.4 \times 0.6-1.1 \mathrm{~mm}$, margin entire or occasionally notched. Ovary sub-spherical to spherical, $0.9-1 \times 0.9 \mathrm{~mm}$, densely pubescent to pilose, hairs straight or primarily straight with some hooked hairs (hooked hairs are shorter and often most easily visible on the fruit operculum), $0.1-0.6(-1) \mathrm{mm}$ long, on very upper portion of ovary; style 2.9-4.2 $\mathrm{mm}$ long, usually glabrous, occasionally 1 or 2 hooked hairs on base of style, $0.2 \mathrm{~mm}$ long; stigma 0.4 mm wide. Fruit cylindrical, ovate or obovate, 2.1-2.3 × 1.7-1.9 mm; operculum 0.6 mm long, pubescence as on ovary. Seed narrowly ovoid to ovoid, $0.3-0.5 \times 0.1-0.2$ $\mathrm{mm}$, medium brown, pattern spiralled and reasonably even.

Distribution. Indonesia (Sulawesi, Maluku (Buru, Seram), West Papua), Philippines.

Habitat and ecology. Lithophytic on both limestone and other rocks, but also occasionally terrestrial. Frequently found on stones and boulders in or beside streams 
and rivers, but also in rock crevices in humid, shady, if not wet, places. Collected from 0-1600 m altitude.

Provisional IUCN conservation assessment. Least Concern (LC). This species is common and very widespread. However, as it frequently occurs on karst limestone, which is often commercially exploited, its status should be monitored.

Additional specimens examined: INDONESIA: Maluku: Buru, NW Buru, Bara, Waeduna River, 30 Nov 1984, Nooteboom, H.P. 5357 (L); Buru, Nov 1984, Sukendar SK740 (BO); Maluku, Buru, Ehoe, 27 Sep 1921, Toxopeus, F.J. 358 (BO); Buru, Waeha, 12 Nov 1984, van Balgooy, M.M.J. 4712 (E, L); Halmahera, Kampong Goal, Idjan 175 (BO); ibidem, 14 Sep 1951, Pleyte, D.R. 150 (BO, L); Seram, Tehoru, Saunulu, Murkele Ridge, 19 Feb 1985, Kato, M. et al. C.6908 (L); Seram, Roho, 21 Nov 1917, Kornassi 482 (BO). Papua: Chaban, 28 Feb 1940, Kanehira, R. \& Hatusima, S. 11848 (A, K). Papua Barat: Raja Ampat, Misool, Near Waima, 26 Sep 1948, Pleyte, D.R. 1053 (BO); Vogelkop Peninsula, Aitinjoe, 11 May 1958, Versteegh, C. BW7405 (L); Biak to Parieri, 11 Sep 1966, Kostermans, A.J.G.H. \& Soegeng 914 (BO, L); Biak, Kampong Saba, 31 May 1959, de Wilde, J.J.F.E. 1155 (L); Biak, 29 Aug 1915, Feuilletau de Bruyn, W.K.H. 335 (BO); Manokwari, Arfak Mts, Minjambau, 22 May 1962, Versteegh, C. BW12694 (BO, L); Manokwari, Arfak Mts, Mupi Dessa, 16 Apr 1995, Sands, M.J.S. 6841 (E, K); Manokwari, Arfak Plains, 25 Apr 1994, Sands, M.J.S. 6337 (E, K); ibidem, 24 Apr 1994, Sands, M.J.S. 6318 (K); Manokwari, Kebar Valley, Andjai Airstrip, Sobor Hills, 15 Nov 1954, van Royen, P. 5063 (A, E, L); Manokwari, Kebar Valley, Andjai Village, 5 Nov 1954, van Royen, P. 4946 (A, K, L); Manokwari, Mt Krabo, 3 Nov 1960, Koster, C. BW10773 (A, L); Manokwari, Warmare, Mokwam Trail, 10 Apr 1994, Sands, M.J.S. 6141 (K); Sorong, Warsamson Valley, 17 Aug 1961, Schram, F.A.W. BW13259 (L); Wandammen Peninsula, Wondiwoi Mountains, 28 Feb 1962, Koster, C. BW13665 (L); ibidem, 24 Feb 1962, Schram, F.A.W. BW10631 (L). Sulawesi: s.l., 1871, Riedel s.n. (P). Sulawesi Tengah: Gunung Nokilalaki, 5 May 1975, Meijer, W. 10045 (BO, L, US); Donggala, c. 57 km north of Palu on Palu-Oti Road, 3 Jun 1979, van Balgooy, M.M.J. 3600 (L). Gorontalo: 9 Apr 2002, Mendum, M. et al. 43 (E); Pinogu to Tulabolu, 15 Apr 2002, Mendum, M. et al. 113 (BO, E); Gunung Boliohutu, Mendum, M. et al. 180 (BO). Sulawesi Tenggara: Kesali-Porema, 23 Oct 1929, Kjellberg, G.K. 2616 (BO, S). Sulawesi Utara: G. Klabat, Pinili, 31 Oct 1973, Dransfield, J. 3869 (BO); Sangihe Island, Tamako, Kentuhang, 23 Nov 1998, Hicks, D. 151 (K).

PHILIPPINES: Agusan el Norte: Cabadbaran, Mt Urdaneta, Aug 1912, Elmer, A.D.E. 13470 (BO, E, G, GH, K, L, NY, P, US). Bohol: Aug 1923-Oct 1923, Ramos, M. BS43313 (BO, G, K, P, US). Bukidnon: Jul 1913-Aug 1913, Escritor, L. BS21452 (US); Mt Galintan, Ramos, M. \& Edaño, G. BS48897 (NY). Cagayan: Penablanca, Mar 1929-May 1929, Ramos, M. BS76791 (GH, SING). Davao del Sur: Sep 1911-Oct 1911, Weber, C.M. 1440 (E, NY, P). Davao Oriental: Baguan River, 8 Mar 1949, Edaño, G. 758 (A, PNH); ibidem, 8 Mar 1949, Edaño, G. 746 (PNH); Davao, Mati, Mar 1927-Apr 1927, Ramos, M. \& Edaño, G. BS49227 (NY); Davao, Mount Bilbogan, 1949, Edaño, G. 1965 (A, L, PNH). Davao del Norte: Mt McKinley, 14 Aug 1946, Edaño, G. 720 (PNH). Ifugao: Feb 1913, McGregor, R.C. BS19951 (US). Isabela: Barangay San Jose, 7 Mar 1997, Argent, G.C.G. et al. 29038 (E). Laguna: Los Banos, Apr 1906, Elmer, A.D.E. 8318 (BO, E, G, K, NY); ibidem, 18 Dec 1903, Hallier, H.G. 4128 (L); ibidem, 7 Mar 1906, Merrill, E.D. 5106 (BO, K, NY, US); ibidem, 14 Jan 1905, Williams, R.S. 2046 (NY); Mt Makiling, Jun 1917-Jul 1917, Elmer, A.D.E. 18283 (BO, GH, K, US); ibidem, Jun 1917-Jul 1917, Elmer, A.D.E. 17551 (BO, GH); ibidem, Jan 1913, 
Robinson, C.B. BS17216 (US). Lanao del Sur: Lake Lanao, Camp Keithley, Sep 1906-Oct 1906, Clemens, M.S. 724 (US). Leyte: Wenzel, C.A. 231 (G, US); ibidem, 28 Jun 1913, Wenzel, C.A. 437 (GH); Mt Abucayan, Feb 1923, Edaño, G. BS41748 (G, K, US). Negros Oriental: Cuernos de Negros, Guinsuan Creek, 6 Jun 1948, Edaño, G. 435a (A); ibidem, 6 Jun 1948, Edaño, G. 435 (PNH); Dumaguete, Cuernos Mts, Apr 1908, Elmer, A.D.E. 9698 (BO, E, G, L, NY, US); Lake Balinsasayo, 16 Sep 1948, Edaño, G. 418 (A, PNH). Oriental Mindoro: Subaan River, 14 Apr 1986, Coode, M.J.E. 5313 (K, L). Palawan: Mt. Kabangaan, Apr 1929, Edaño, G. s.n. (GH). Quezon: Mt Cristobel, 14 Dec 1996, Reynoso \& Majaducon PPI27265 (K). Rizal: Morong, 25 Mar 1893, Loher, A. 4231 (K); Mt Angilog, Feb 1923, Lopez, G. BS42049 (BO, L, P, US); ibidem, Feb 1923, Lopez, G. BS42031 (US); Mt. Lumutan, Apr 1923, Ramos, M. 42176 (BO). Samar: Matuguinao, Baruz, 20 Jan 1952, Gachalian, F. 147 (A, PNH); Mt Purog, 24 Dec 1951, Edaño, G. 3478 (A); Mt Sarawag, 3 Dec 1951, Edaño, G. 3070A (PNH). Sorsogon: Irosin, Mt Bulusan, Apr 1916, Elmer, A.D.E. 15877 (BO, C, G, GH, K, L, NY, P, US); ibidem, 1915, Elmer, A.D.E. 15626 (BO, GH, NY). Zamboanga: Nov 1911-Dec 1911, Merrill, E.D. 8222 (US); Sax River, 18 Feb 1905, Williams, R.S. 2401 (NY).

Notes. There are two informal forms of Epithema benthamii. The typical form is found throughout the distribution of the species, while a finer, more thinly membranous and more elongate form is found on Buru Island (Maluku) and Biak Island off Papua (e.g. Sands 6318). These plants are similar to Epithema longipetiolatum, but still have the typical leaf form and the characteristic long, straight or primarily straight, hairs on the ovary and operculum of E. benthamii. The distributions of Epithema benthamii and E. longipetiolatum overlap on Buru Island and Biak Island. See further discussion under Epithema longipetiolatum. The indumentum of the leaf is useful for identification of Epithema benthamii in that there are no minute hairs interspersed between the larger hairs. The strigose hairs are often white, clearly visible and distinctive on the surface of the dried leaves.

The specimen Kanehira and Hatusima 11848 (A) is unusual in that the peduncle is branched and racemosely bears many complete inflorescences.

In the type specimen of Epithema brunonis var. scabrida the leaves are somewhat dentate rather than entire as stated in the protologue, the indumentum is of the same form as Epithema benthamii, albeit slightly denser, and the hairs on the ovary/operculum are long and straight as in E. benthamii rather than the hooked hairs of E. brunonis. It is quite clearly a synonym of Epithema benthamii.

The only apparent difference between Epithema calcicola and E. benthamii is in the narrower somewhat reflexed bracts of E. calcicola. This species was known only from the type collection and we conclude it is only a rather unusual specimen of Epithema benthamii based on all other characters and that it occurs within the distribution of this species.

2. Epithema carnosum Benth., Scroph. Ind. 57 (1835); Barnett, Fl. Siam. 3(3): 205 (1962), p.p.; Wang et al., Fl. China 18: 400 (1998); B.L.Burtt, Thai For. Bull. (Bot.) 29: 93 (2001). - TYPE: Nepal, Nag Argute, 1832, Wallich, N. s.n. (lectotype K [K000438688], designated here). (Fig. 3) 


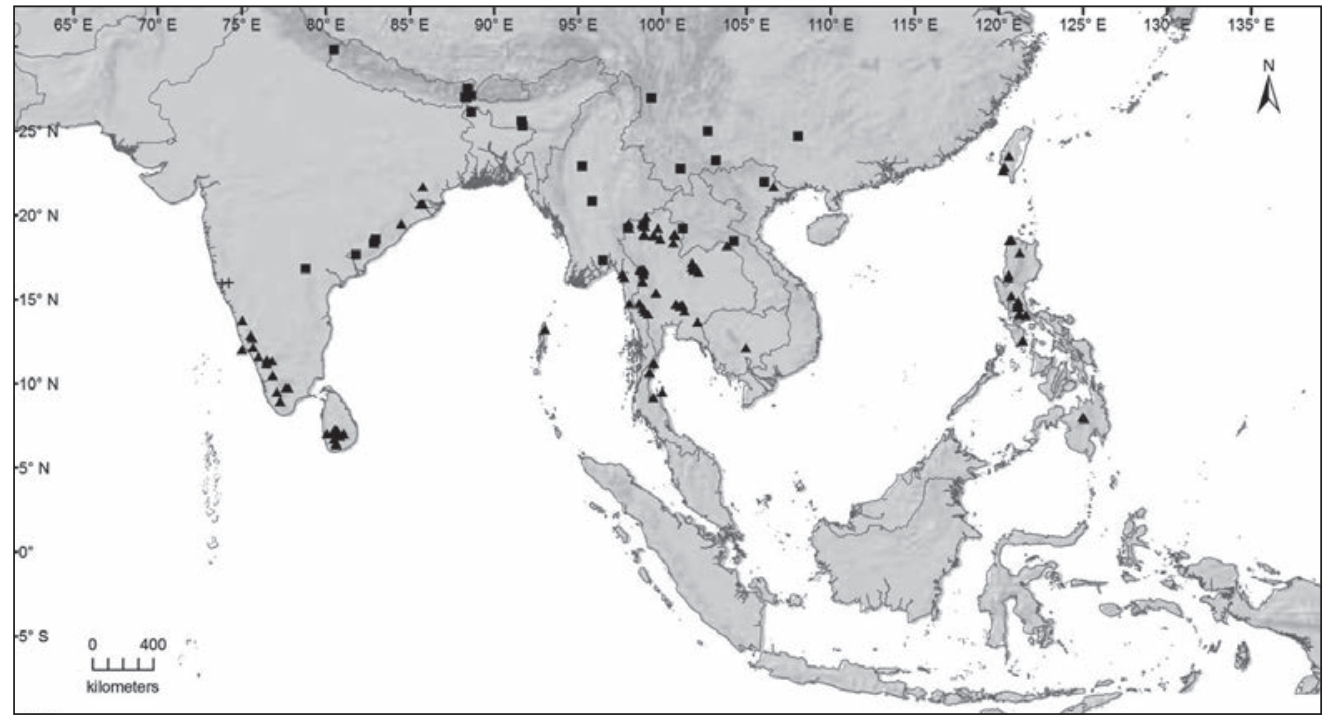

Fig. 3. Distribution of Epithema carnosum Benth. (घ), Epithema ceylanicum Gardner ( $\mathbf{\Delta})$ and Epithema pusillum (C.B.Clarke) Bransgrove (+).

Herb 6-38 cm high, caulescent; stem 1.5-5 mm wide with 2-3 nodes, internodes 3-15 cm long, usually sub-glabrous to strigose or pubescent, rarely densely so. Leaves thinly membranous to membranous, lower leaf petiolate, upper leaves petiolate or sessile; petioles of lowest leaf 5-7.5 cm long, upper petioles 0-7 cm long; blades of the lowest leaf $6.5-17 \times 6-12.5 \mathrm{~cm}$, upper leaves $2.5-12.5 \times 2.3-6 \mathrm{~cm}$, sub-spherical, cordate or often ovate, symmetrical or barely asymmetrical, apex acute to broadly acute, occasionally rounded, base sub-cordate to truncate, inserted evenly on petiole or not, margin often sub-entire, but also serrate, dentate or crenate-sinuate (especially upper leaves); hairs on upper surface to $1.2 \mathrm{~mm}$ long; lower surface whitish-green or paler than upper surface. Inflorescences 1-12 per plant; peduncles $0.5-7 \mathrm{~cm}$ long, arising from leaf axils, from the petioles or from the leaf midribs, primarily from the upper leaves; bracts small or sub-cucullate, enclosing a small portion of the inflorescence, $1-13 \times 3-9 \mathrm{~mm}$, margin roughly entire with few dentate teeth to more dentate; pedicels 1-5 mm long. Calyx light or whitish-green, 3.3-5.4 mm long; tube 2-3.3 × 1.5-2.3 mm; lobes 1.3-2.1 × 0.7-1 mm; indumentum outside a minute hooked pubescence under strigose hairs $0.25-0.5 \mathrm{~mm}$ long. Corolla blue to dark purple, some with purple spots on the lower lip, 5.5-7.5 mm long; tube (3-)4-5.5 × (1-)1.5-2 mm long; lobes 1-2.3 mm long; glabrous outside, villous band of hair inside. Stamen filaments $0.6-0.8 \mathrm{~mm}$ long; anther dimensions unknown; staminodes $0.6-0.8 \mathrm{~mm}$ long. Nectary absent, of one lobe almost encircling ovary, or of two lobes, undulate, each end a distinctly different height from the other, 0.6-1 × 1.1-1.8 mm. Ovary subspherical, $0.5-0.83 \times 0.4-0.75 \mathrm{~mm}$, pubescent or pilose, medium to high density, hairs straight or primarily straight, hyaline, usually $0.1-0.25 \mathrm{~mm}$ long, occasionally to 0.4 
mm long, on upper area of ovary; style c. $4 \mathrm{~mm}$ long, glabrous to pubescent, hairs sparse to high density, to one third or half way up style, hairs hooked, hyaline, to $0.1 \mathrm{~mm}$ long; stigma c. $0.5 \mathrm{~mm}$ wide. Fruit sub-cylindrical $2.1-2.5 \times 1.7-2.5 \mathrm{~mm}$; operculum c. $0.6 \mathrm{~mm}$ long, indumentum as ovary. Seed oval to fusiform, ends acute to constricted, $0.4-0.6 \times 0.1-0.2 \mathrm{~mm}$, medium brown, pattern often straight to more or less straight, but also to spiralled.

Distribution. India, Nepal, China, Myanmar, Thailand, Laos, Vietnam.

Habitat and ecology. Lithophytic. Found in humid, shaded places, often near water and in rocky crevices at 0-2120 m altitude. Flowering and fruiting April, July-October.

Provisional IUCN conservation assessment. Least Concern (LC). This species is very widespread. However, as it frequently occurs on karst limestone, which is often commercially exploited, its status should be monitored.

Additional specimens examined. INDIA: Andra Pradesh: Chintapalli, 31 Aug 1966, Subba Rao, G.V. 28210 (E); Godavari, Rampa Country, Devarakonda, 6 Oct 1920, Narayanaswami, M.A. 478 (CAL); Visakhapatnam, Araku, 24 Aug 1960, Balakrishnan, N.P. 10815 (BSI, CAL, E). Meghalaya: Khasia, 28 Oct 1872, Clarke, C.B. 19076 (US); Khasia, Cherrapunjee, 26 Aug 1850, Hooker, J.D. \& Thomson, T. 2161 (K); Khasia, Sohra, 22 Oct 1871, Clarke, C.B. 15665 (L); Mont. Khasia, Hooker, J.D. \& Thomson, T. s.n. (C, CAL, E, GH, L, NY, P). Orissa: Koraput, 8 Oct 1950, Mooney, H.F. 4045 (K); Koraput, Pottangi, 10 Jul 1950, Mooney, H.F. 3853 (K); ibidem, 13 Jul 1950, Mooney, H.F. 3891 (K); Koraput, Turia Konda, 10 Oct 1950, Mooney, H.F. 4092 (K); Sikkim, 1878, King, G. s.n. (CAL); ibidem, Thomson, T. s.n. (BO, L); ibidem, Unknown 3847 (K); Sikkim, Mongpo, 5 Oct 1884, Clarke, C.B. 36192 (G, K). Uttaranchal: Kumaon, 25 Sep 1884, Duthie, J.F. 2990 (K). West Bengal: Darjeeling, Unknown 12200 (BO); Darjeeling, Mungpoo, 23 Sep 1875, Clarke, C.B. 24805 (K); Darjeeling, Rishap, 2 Aug 1870, Clarke, C.B. 12300 (CAL, L); Darjeeling, Rishap, Rishap Ravine, 4 Sep 1869, Clarke, C.B. 8997 (L).

CHINA: Heou-hay tse, 7 Jul 1907, Esquirol, J. 711 (E). Guangxi: Tan-Ngar, 12 Jul 1928, Ching, R.C. 6421 (A). Yunnan: Shweli-Salwin Divide, 1917-1919, Forrest, G. 15699 (E, K); Simao, Henry, A. 13497 (K, US); ibidem, Henry, A. 12280 (K); ibidem, Henry, A. 12705 (K). MYANMAR: Pegu, Scott 3 (L); Mandalay, Meiktila, Taungbaw-yo, 22 Oct 1936, Smith, H.C. 16273 (K); Mandalay, Myingyan, Pope Hill, 4 Aug 1909, Lace, J.H. 4892 (E, K).

THAILAND: Chiang Mai: Chiang Dao, Doi Chiang Dao, 22 Jul 1989, Maxwell, J.F. 89-920 (L); ibidem, 27 Sep 1971, Murata, G. et al. 15146 p.p. (C, K, KYO); ibidem, 27 Sep 1971, Murata, G. et al. T.15156 (KYO); ibidem, 19 Oct 1926, Put 410 (K); ibidem, 26 Oct 1979, Shimizu, T. et al. T.20909 (KYO). Mae Hong Son: Muang, Doi Mae Sakut, 23 Sep 1995, Nanakorn, W. et al. 4664 (E). Nan: Sapan Waterfall, 7 Sep 1995, Larsen, K. et al. 46161 (AAU). Tak: Nam Tok Phacharoen National Park, 23 Apr 2004, Pooma, R. et al. 4580 (BKF). LAOS: Sayaboury: Phiang, Nam Phon, 1 Sep 1999, Maxwell, J.F. 99-278 (A, L).

VIETNAM: Bac Kan: Na Ri District, Liem Thuy Municipality, Na Bo Village, 15 Jul 2004, Atha, D. 4785 (NY); Ha Giang: Pho Bang, Pho Cao, 12 Aug 1977, Phuong, V.X. 311 (HN).

Notes. Epithema carnosum is widespread across tropical Asia with the exception of Malesia. It is similar to some of the forms of Epithema ceylanicum and was originally 
separated from it (as E. dentatum) by E. carnosum having crenate-sinuate leaves vs. $E$. dentatum having small teeth on the leaf margin that are sometimes subacute or doubly serrate. Also Epithema dentatum was said to have shorter petioles on the upper leaves. These characters cannot consistently be used to separate Epithema carnosum from the now more broadly defined E. ceylanicum which includes E. dentatum. It is better separated from Epithema ceylanicum by the hairs on the ovary and operculum. They are straight in E. carnosum and hooked or primarily so in E. ceylanicum. In nearly all specimens from India, Nepal and Myanmar there is no variation in this character, but on specimens from China, Thailand and Vietnam a proportion of the hairs on the ovary/operculum of Epithema carnosum may be hooked. In all cases where the majority of the hairs are straight, the specimens are assigned to E. carnosum. This is also essentially true of Epithema ceylanicum where the hairs on almost all specimens from India and Myanmar have only hooked hairs on the ovary and operculum but some specimens from north and north-west Thailand also have some straight hairs on the ovary/operculum. In all cases where the majority of the hairs are hooked, the specimens are assigned to E. ceylanicum.

Epithema carnosum occasionally produces peduncles from the petioles of the leaves of the terminal leaf pair. Peduncles arising from the midrib of these leaves has only been observed in specimens of Epithema carnosum from Khasia in Northeast India.

3. Epithema ceylanicum Gardner, Calcutta J. Nat. Hist 6: 492 (1846). - Epithema carnosum var. ceylanicum (Gardner) C.B.Clarke in A.DC. \& C.DC. Monogr. Phan. 5(1): 178 (1883). - TYPE: Sri Lanka, Gardner, G. 606 (lectotype K, designated here). (Fig. 3-5)

Epithema carnosum var. hispidum C.B.Clarke in A.DC. \& C.DC., Monogr. Phan. 5(1): 178 (1883). - Epithema dentatum subsp. hispidum (C.B.Clarke) Hilliard \& B.L.Burtt, Edinburgh J. Bot. 54: 112 (1997). - TYPE: India, Tamil Nadu, Western Ghats, Courtallum, August 1835, Wight, R. 2350 (lectotype K, designated here)

Epithema carnosum var. dentatum C.B.Clarke in A.DC. \& C.DC., Monogr. Phan. 5(1): 177 (1883). - Epithema dentatum (C.B.Clarke) Hilliard \& B.L.Burtt, Edinburgh J. Bot. 54: 111 (1997). - TYPE: Burma, Mon, Moulmein, Farm Cave Rocks, Parish, C.S.P. 63 (lectotype K, designated by Hilliard \& Burtt (1997)).

Epithema brunonis var. fasciculatum C.B.Clarke in A.DC. \& C.DC., Monogr. Phan. 5(1): 180 (1883). - Epithema taiwanense var. fasciculatum (C.B.Clarke) Z.Yu Li \& M.T.Kao, Fl. Taiwan ed. 2, 4: 697 (1998). - TYPE: Philippines, Tayabas, 1841, Cuming, H. 823 (lectotype K [K000438698], designated here; isolectotype BM, G-DC, K, L, P). 


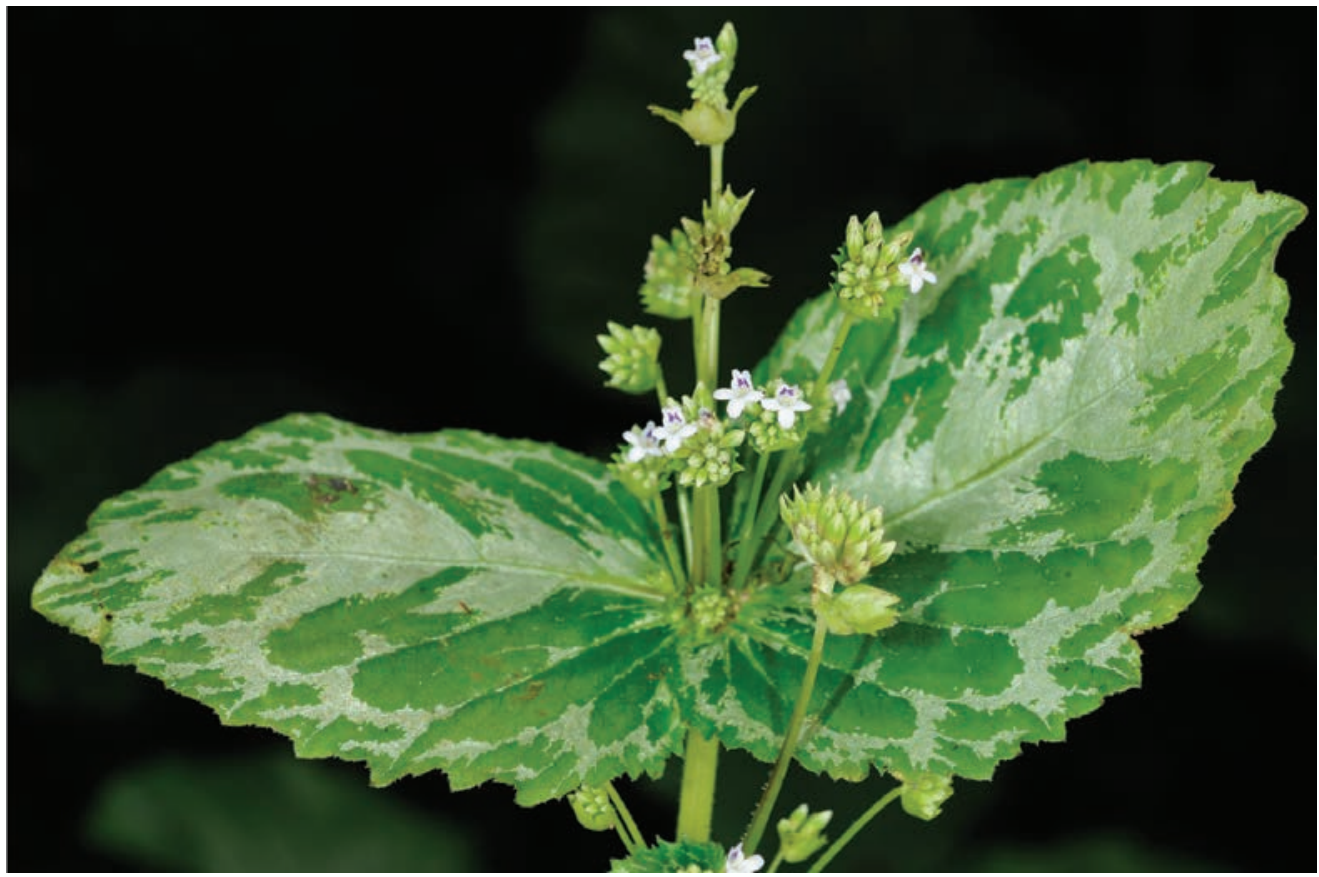

Fig. 4. Epithema ceylanicum Gardner. Leaves and inflorescences. (Photo: Preecha Karaket)

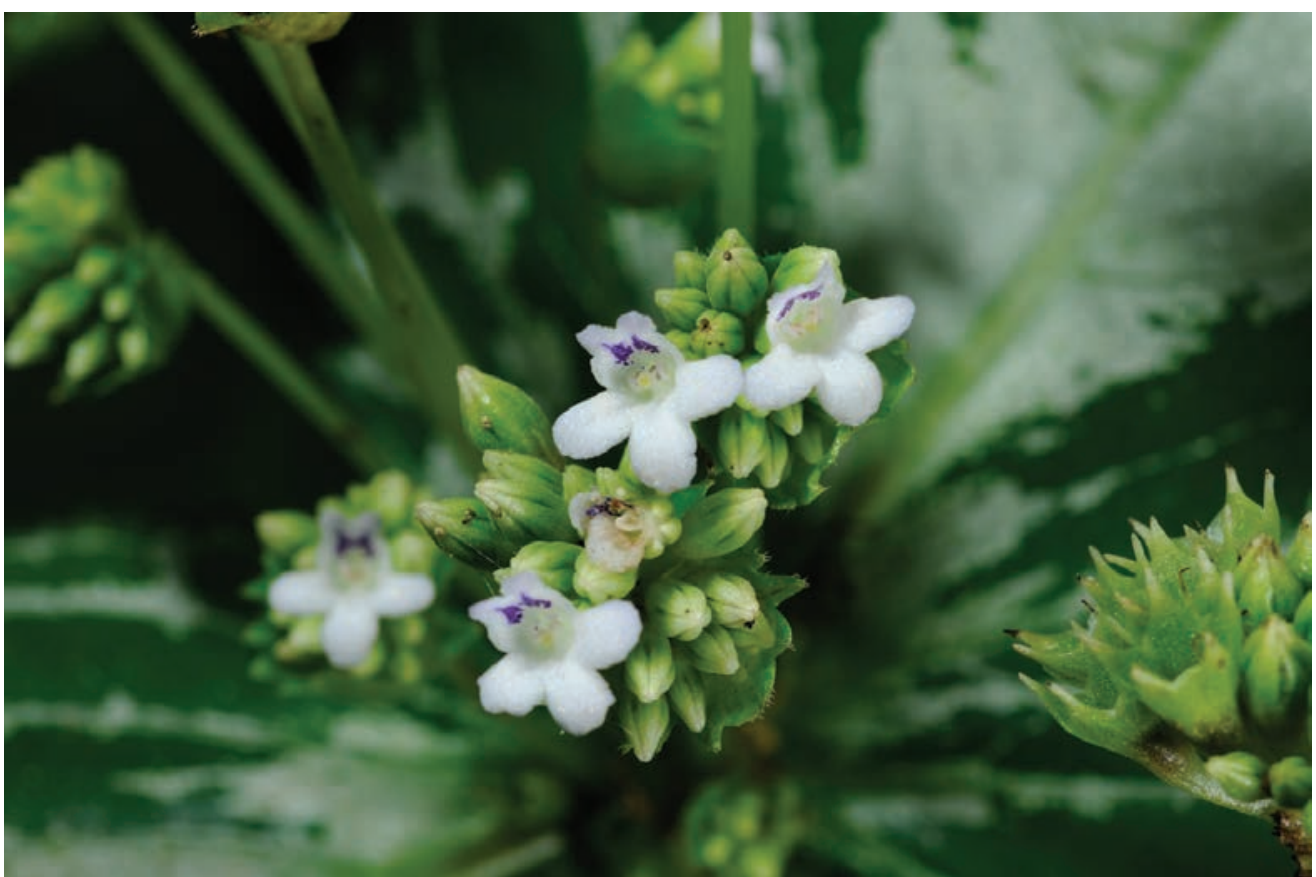

Fig. 5. Epithema ceylanicum Gardner. Flowers. (Photo: Preecha Karaket) 
Epithema taiwanense S.S.Ying, Coloured Ill. Fl. Taiwan 4: 795 (1992); Li \& Kao, Fl. Taiwan ed. 2, 4: 697 (1998); Wang et al., Fl. China 18: 400 (1998). - TYPE: Taiwan, Chia-yi, Kuanyin waterfalls, 200-300 m, 25 June 1992, Ying, S.S. s.n. (holotype NTUF n.v.).

Herb 4-40 cm high, caulescent or with very short primary internode and appearing acaulescent; stem 1-10 mm wide, internodes 0.5-15 cm long, sub-glabrous, sparsely strigose or sparsely pubescent, hairs $0.5-1 \mathrm{~mm}$ long. Leaves thinly to strongly membranous, lowest leaf always petiolate, upper leaves sessile to petiolate, sometimes with a solitary upper, sessile leaf per plant; petiole of lowest leaf (0.2-)1-12 cm long, sub-glabrous to densely pubescent, hairs to $0.7 \mathrm{~mm}$ long, petioles of upper leaves $0-2$ cm long, sub-glabrous, minutely pubescent or with longer hairs of medium to high density, to $1 \mathrm{~mm}$ long; blades of lowest leaves 3-20 × 1.8-20 cm, upper or solitary leaves $(1-) 1.5-11 \times 0.8-7 \mathrm{~cm}$, lower leaves cordate to ovate, upper leaves cordate, ovate or elliptic, occasionally almost oblong or sub-spherical, symmetrical or asymmetrical, apex acute to rounded, base of lower leaf cordate to truncate, base of upper leaves obtuse, rounded or truncate, occasionally sub-cordate, inserted evenly on petiole or not, where inserted unevenly one side is often cordate, the other truncate, margin subentire to dentate, bidentate or serrate; upper surface pale- to mid-green, with sparse straight and/or hooked hairs to $0.9 \mathrm{~mm}$ long and sparsely to densely strigose, hairs 0.25-1.5 mm long; lower surface paler green or purplish in colour, sub-glabrous to strigose, hairs sparse to medium density, sometimes with hooked and straight hairs on veins to $0.3 \mathrm{~mm}$ long. Inflorescences $1-6$ (to many) per plant; peduncles arising from the leaf axil, the petiole or the base of the midrib, usually from the upper leaves, often rather fasciculate, $(0.1-) 1-10(-17) \mathrm{cm}$ long, often of very variable lengths in a single cluster, sub-glabrous to densely setose, hairs to $1.1 \mathrm{~mm}$ long; bracts (sub-)cucullate but enclosing a small proportion of the inflorescence if at all, 3-16(-20) $\times 3-9(-10)$ $\mathrm{mm}, 0.6-2(-3.4)$ times as long as wide, margin entire to partially or entirely dentate; lower surface glabrous to strigose, hairs sparse to medium density, hairs $0.25-0.8 \mathrm{~mm}$ long, with or without shorter layer of hair $0.1-0.4 \mathrm{~mm}$ long; upper surface glabrous to sparsely strigose, hairs to $0.25 \mathrm{~mm}$ long, hairs on upper half of bract or towards margins; pedicels $0.5-6 \mathrm{~mm}$ long, densely pubescent with fine minute hairs and more sparsely with larger strigose hairs to $1 \mathrm{~mm}$ long. Calyx 2.1-8 mm long; tube 0.8-6 × 1-2.9 mm; lobes 1-2.5 $\times 0.7-2.1 \mathrm{~mm}$; sub-glabrous or sparsely to densely pubescent outside with fine, minute straight and hooked hairs to $0.25 \mathrm{~mm}$ long, with or without sparse, strigose hairs, to $0.4 \mathrm{~mm}$ long; glabrous or subglabrous inside, sometimes with strigose hairs on inside of lobes. Corolla whitish or blue, blue-violet or pink, tube white, 5-8.5 mm long; tube 3.2-6.3 × 1-3 mm; lobes 1-3 × 1-1.3 mm long, margins often slightly fimbriate; glabrous to sparsely pubescent outside on lobes, hairs straight; dense band of villous hair inside, one-third to three-quarters way up the tube from the base. Stamens 1.4-2.1 mm long; filaments 1-1.7 mm long; anthers $0.4-0.5 \mathrm{~mm}$ long; staminodes 1-1.7 mm long. Nectary apparently absent or one or two discrete lobes, may almost encircle ovary or not, margin entire or sub-entire, undulate or notched, 0.8-1.8 × 1-1.8 mm. Ovary sub-spherical to cylindrical, 0.7-1.5 × 0.9-1.5 mm, 
sparsely to densely pubescent on the upper half or uppermost part of the ovary, hairs hooked or primarily hooked, hyaline, to $0.15 \mathrm{~mm}$ long; style $2.5-5 \mathrm{~mm}$ long, glabrous or sparsely pubescent in lower half, hairs hooked; stigma to $0.5-0.8 \mathrm{~mm}$ wide. Fruit spherical to cylindrical, $1.5-5 \times 1-3 \mathrm{~mm}$; operculum $0.4-0.8 \mathrm{~mm}$ long, sparsely to densely pubescent as on ovary. Seed sub-cylindrical to ovoid, $0.4-0.7 \times 0.1-0.2 \mathrm{~mm}$, pattern often straight or almost straight, but also to spiralled, regular, walls clearly defined, often appearing thickened and rigid.

Distribution. India (including Andaman Islands), Sri Lanka, Taiwan, Myanmar, Thailand, Cambodia, Vietnam, Philippines.

Habitat and ecology. Lithophytic. Found on rocks and rock faces in wet, humid, shady places, occasionally on wet tree trunks at 0-2000 m altitude.

Provisional IUCN conservation assessment. Least Concern (LC). This species is very widespread. However, as it frequently occurs on karst limestone, which is often commercially exploited, its status should be monitored.

Additional specimens examined. INDIA: Andaman and Nicobar Islands: North Andaman, Saddle Peak National Park, 10 Feb 2000, Sumathi, R. 17895 (K). Karnataka: Chorla Ghats, Chorla, Stocks s.n. (K); Coorg, Mercara, Jersey Falls, 12 Sep 1934, Barnes, E. 928 (K); Mysore, Hassan, Bisle ghat, 14 Aug 1971, Ramamoorthy, T.P. HFP2007 (US); Mysore, Hassan, Shiradi ghat, 7 Aug 1969, Saldanha, C.J. 14426 (US); Mysore, Kenchankumri State Forest, 15 Aug 1971, Ramamoorthy, T.P. HFP2035 (K, US); Mysore, Shimoga, Hulical ghat, 24 Aug 1963, Raghavan, R.S. 90185 (E). Kerala: Idikki District, Sabarimalai slopes, 26 Sep 1972, Sharma, B.D. 42032 (E); Kochi (Cochin), Parambikulam, 25 Sep 1935, Barnes, E. 1300 (K); Kochi (Cochin), Parambikulam Hills, Dec 1934, Barnes, E. 1056 (K); Palghat, Silent Valley, 21 Aug 1966, Vajvavelu, E. 27558 (L). Madhya Pradesh: Bastar, Upper Kanger Valley, 26 Aug 1959, Subramanyam, K. 8644 (E). Maharastra: Concan, Hooker, J.D. \& Thomson, T. s.n. (P); ibidem, Stocks, Law \& co. s.n. (C, L). Orissa: Dhenkanal State, Goyalpathar ghati valley, 16 Sep 1942, Mooney, H.F. 2067 (K); Dhenkanal State, Kapilas Hill, Mooney, H.F. 2754 (K); Dhenkanal State, Saptasajya, 20 Sep 1993, Dhal, N.K. \& Rout, N.C. 8122 (E); Ganjam, Budhakhol Pahad, 14 Aug 1971, Brahmam, M. 2776 (E). Tamil Nadu: Banliyar, ?Wight s.n. (L); ibidem, ?Wight s.n. (L); Madurai, Way, 14 Oct 1959, Subramanyam, K. 8975 (E); Mont. Nilghiri \& Kurg, Thomson, G. 121 (K); ibidem, Thomson, G. s.n. (E, G); Vilangadu Forest, 27 Aug 1985, Pradeep, A.K. 5850 (CAL); Western Ghats, Courtallum, W. C. 2680 (E); ibidem, Wight, R. 556 (K); Western Ghats, Courtallum, Swamiar Falls, 2 Oct 1975, Nair, K.K.N. 1354 (CALI). West Bengal: Kolcata, Kabini tributory falls, 17 Aug 1964, Ellis, J.L. 20452 (E).

SRI LANKA: s.l., 1891, Deschamps s.n. (G); Mrs. General Walker 140 (K); Mrs. General Walker s.n. (K); Thwaites, G.H.K. 2844 (BO, G, K, P); 1836, Wight, R. 1/162 (K). Uva: Badulla District, Between Mesitale and Dunhinda Falls, 26 Jan 1982, Namba, T. \& Mikage, M. 82413 (KYO); Badulla District, Dunhinda Falls, 8 Jan 1983, Lucas 1025 (US). Central: Matale District, Wiltshire Forest, 2 Jul 1974, Sumithraarachchi, D.B. DBS388 (K); Deltotte, Nov 1888, Unknown s.n. (SING); Kandy, Laksapana, 19 Sep 1972, Jayasuriya, A.H.M. 843 (US); Peradeniya, 10 Sep 1934, Unknown 753 (L). Sabaragamuwa: Kegalle District, Dolosbage, Windsor Forest, 17 Nov 1978, Grey-Wilson, C. \& Grey-Wilson, C.M. 3016 (K, US); Kegalle District, Kadaganawa, 1 Sep 1969, Grupe, D.A. 200 (US); ibidem, 22 Aug 1968, Theobald, 
W.L. \& Grupe, D.A. 2404 (US); ibidem, 30 Jul 1968, Theobald, W.L. \& Grupe, D.A. 2368 (US); Ratnapura, Katussagala Hill, 5 Dec 1976, Faden, R.B. \& Faden, A.J. 76/494 (US); Ratnapura, Pannil Kanda, 5 Jul 1975, Waas, S. 1407 (K); Ratnapura, Rakwana, 7 Dec 1977, Fosberg, F.R. 57278 (K, US).

TAIWAN: Takow, Ape’s Hill, Henry, A. 1916 (E, K, NY, US); Kaohsiung Hsien, Shuangchi, Meilong, 31 Oct 1988, Hsieh, C.F. et al. s.n. (E); Kaohsiung Hsien, Takangshan, 14 Nov 1988, Yang, K.C. 3465 (E).

MYANMAR: Mon: Moulmein, Jul 1880, Brandis, D. 676 (K); ibidem, Lobb, T. 387 (K); ibidem, McKee, H.S. 1958 (P); ibidem, Parish, C.S.P. 133 (K); Moulmein, Kyauk Ta Lon, Jul 1958, McKee, H.S. 6317 (K, P).

THAILAND: Bueng Kan: Mueang, Chaiyaphon, Singhanat Ban Phot Temple, 26 Aug 2001, Pooma, R. et al. 2697 (BKF). Chiang Mai: Chiang Dao, Pooma, R. 1059 (BKF); ibidem, 16 Jul 1958, Smitinand, T. 4719 (BKF); ibidem, 15 Aug 1963, Smitinand, T. \& Sleumer, H.O. 1006 (BKF); Chiang Dao, Doi Chiang Dao, 27 Sep 1971, Murata, G. et al. 15146 p.p. (L); ibidem, 17 Jul 1958, Sorensen, T. et al. 4235 (A, BKF, C, E); Chiang Dao, Doi Chiang Dao, Between Pong Pho and Khun Klong, 31 Jul 1968, Larsen, K. et al. 2923 (AAU); Doi Sutep, 10 Oct 87, Maxwell, J.F. 87-1154 (BKF, L); ibidem, 12 Sep 1958, Sorensen, T. et al. 4936 (ABD, C); Mae Ram Subdistrict, Doi Sutep-Pui National Park, Mae Ram Stream, 10 Sep 1992, Palee, P. 73 (A, CMU, GH, L); Fang, Doi Ang Khang, 21 Sep 2008, Middleton, D.J. et al. 4544 (E); Mae Dang, Mawn Ngaw, Doi Mawn Ngaw, 12 Aug 2002, Palee, P. 544 (L). Chumphon: Sapli, 8 Sep 1927, Put 1015 (ABD, K). Kanchanaburi: 15 Aug 1968, Nimanong, B. \& Phusomsaeng, S. 288 (BKF); ibidem, Suvanakoses, P. 2060 (BKF); Kanburi, Baw Re, 21 Jul 1926, Put 219 (K); Sai Yok, Sai Yok National Park, Lam Tam Keio, 10 Aug 1982, Shimizu, T. et al. T.28519 (BKF, SING); Sangkhlaburi, 28 Jul 1968, Prayad 1422 (BKF); Thong Pha Phum, 4 Jul 1973, Maxwell, J.F. 73-107 (AAU, BK); Wang Pho, 26 Oct 1969, Kasem 641 (BKF); Khon Kaen, Chum Phae, Phu Pha Man national park, Tham Pha Puang, 1 Aug 2011, Norsaengsri, M. et al. 7965 (QBG); Khon Kaen, Nanong Tum, Ban Na Chan, 23 Aug 2011, Norsaengsri, M. et al. 8048 (QBG); Khon Kaen, Pha Nok Khao, 9 Sep 1963, Smitinand, T. \& Sleumer, H.O. 1109 (K, L, SING). Lampang: Doi Pang La, Huay Tak, 25 Sep 1967, Shimizu, T. et al. T.10789 (BKF); Muang Bahn, Chae Son, Jae Sawn Stream, 22 Aug 1995, Maxwell, J.F. 95-536 (BKF, CMU, L); Ngao, Near Tham Pha Thai, 24 Sep 2008, Middleton, D.J. et al. 4579 (E); Loei, Phu Kradung, 15 Oct 1967, Prayad 1034 (BKF). Loei: Phu Ruea District, Phu Luang, 16 Sep 1966, Phusomsaeng, S. \& Bunchuai, K. 42 (BKF, L). Mae Hong Son: Muang, Doi Pui, 23 Sep 1995, Larsen, K. et al. 46861 (AAU); Mueang, Tham Pla-Namtok Pha Suea National Park, Pha Daeng Cave, 22 Aug 2013, Norsaengsri, M. 10568 (QBG). Nakhon Nayok: Nang Rong Falls, 4 Aug 1992, Larsen, K. et al. 43771 (P). Nan: Tham Pha Toop Forest Park, 2 Sep 1999, Middleton, D.J. 149 (A, BKF, E, K); Muang, Tham Phatup Forest Park, 16 Aug 2012, Middleton, D.J. et al. 5613 (E, SING); Muang Tam Paa Toop, 13 Sep 1995, Nanakorn, W. 4240 (QBG); Tham Pha Tok, 25 Jul 1992, Larsen, K. et al. 43589 (AAU); ibidem, 13 Sep 1995, Larsen, K. et al. 46426 (AAU). Phayao: Doi Luang National Park, Champa Thong Waterfall, 9 Aug 1997, Petrmitr, O. 70 (L). Prachuap Khiri Khan: Bang Saphan, Khao Maa Rong, 5 Sep 2008, Middleton, D.J. et al. 4284 (E). Phrae: Nam Pu, 23 Sep 1912, Van Pruk, L. 350 (BK, K). Sa Kaeow: Khao Chakan, 17 Oct 2010, Staples, G.W. et al. 1409 (E, SING). Saraburi: Kaeng Khoi, Amata-khuha Cave, 20 Aug 2001, Pooma, R. et al. 2100 (BKF); Kaeng Khoi, Tharn Pra Photisat, 7 Oct 1979, Shimizu, T. et al. T.19413 (KYO, L); Muak Lek, 10 Nov 1924, Marcan, A. 1869 (ABD, K); ibidem, 3 Sep 1928, Put 1853 (K); Phraphutthabat, Wat Khaowong, 18 Sep 2004, Pooma, R. et al. 4824 (E). Surat Thani: Kanchanadit, 31 Jul 1927, Kerr, A.F.G. 13053 (K); Koh Samui, Kao Noi, 28 Jun 1966, Sakol 1150 (BKF). Tak: Mae Sot, Doi Muser, 20 Aug 
1961, Chermsirivathana, C. 1 (BKF); Mae Sot, Khao Pha Wo, 23 Jul 1973, Murata, G. et al. T.16922 (AAU, BKF, KYO, L); ibidem, 12 Oct 1979, Shimizu, T. et al. T.18519 (KYO); Mae Sot, Wat Tham Inthanin, 11 Sep 2009, Middleton, D.J. \& Triboun, P. 4846 (E). Tak: Umphang, Takhobi Cave, 18 Oct 2014, Middleton, D.J. et al. 5768 (BKF, E, SING). Uthai Thani: Lan Sak, Huppatat Non Hunting Area, 14 Oct 2014, Middleton, D.J. et al. 5687 (BKF, E, SING). CAMBODIA: Kampot: 20 Sep 1903, Geoffray, M. 95 (P).

VIETNAM: Lang Son: Langnac, Eberhardt 3363 (P).

PHILIPPINES: Apayao: May 1917, Fenix, E. BS28106 (US). Benguet: Mt Trail, Oct 1929, Quisumbing, E. BS78105 (NY); Twin Peaks, 8 Sep 1904, Williams, R.S. 937 (NY). Bukidnon: Tanculan, Jul 1916, Fenix, E. BS26049 (K, NY, US). Bulacan: 8 Sep 1935, Bartlett, H.H. 14744 (GH). Ilocos Norte: Bangui, Nov 1923, McGregor, R.C. BS43566 (K); Burgos, Jul 1918, Ramos, M. BS32892 (GH, K, US). Laguna: Los Banos, Loher, A. 1555 (K). Oriental Mindoro: Mansalay, Mt Yagaw, Nov 1952-Dec 1952, Sulit, M.D. \& Conklin, H.C. 16913 (L, PNH). Pampanga: Mt Arayat, Sep 1905, Merrill, E.D. 4216 (K). Rizal: Aug 1911, Ramos, M. BS13624 (BO, G, K, L, P, US). Rizal: Antipolo, Jul 1917, Ramos, M. \& Edaño, G. BS29466 (BO, P, US); Kay Ungulan, 18 Aug 1935, Bartlett, H.H. 15380 (GH); Montalban, 11 Aug 1935, Bartlett, H.H. 14487 (GH, PNH); ibidem, 3 Aug 1990, Loher, A. 1556 (K); ibidem, Dec 1904, Loher, A. 6669 (K); ibidem, Dec 1904, Loher, A. 6668 (K, US); ibidem, 26 Sep 1890, Loher, A. 1554 (K, P, US); ibidem, Nov 1909, Merrill, E.D. 52 (G, US); ibidem, 2 Nov 1909, Robinson, C.B. BS9534 (NY); ibidem, 2 Nov 1909, Robinson, C.B. BS9525 (L, US); Morong, Aug 1906, Ramos, M. BS1435 (NY, US).

Notes. Epithema ceylanicum is a widespread and variable species. The protologue of Epithema ceylanicum states the plants are hispidly pilose, have a persistent style and have five distinct nectaries. Only a proportion of the specimens from Sri Lanka are densely pubescent, only the basal section of the style is persistent and we have seen no more than two nectaries. As a variety Clarke (1883) separated Epithema carnosum var. ceylanicum (as "zeylanica") from E. carnosum var. carnosum on its larger calyx and seeds that are twice as large. However, neither of these characters holds up to closer scrutiny. Instead, it is easily distinguished from Epithema carnosum by the hooked rather than straight hairs on the operculum.

Rarely, it can be difficult to differentiate Epithema ceylanicum from E. saxatile, particularly as they both have hooked hairs on the ovary/operculum. They are most easily distinguished in the size of the bract which in Epithema saxatile usually encloses the inflorescence.

Epithema taiwanense was previously distinguished by the presence of many short, often fasciculate, peduncles with very small inflorescences and quite consistently rounded or rounded-acute leaf apices. However, these characters are within the overall range of variation of the very variable Epithema ceylanicum and we have, therefore, decided to synonymise the names and recognise a single species.

Some specimens bear a resemblance to Epithema involucratum but they can be distinguished by the presence of hair in the corolla and the usually numerous, small inflorescences among other characters. One of the specimens from the Philippines, Fenix BS28106 (US), somewhat resembles Epithema benthamii in leaf shape but is, again, easily separated by having hooked hairs on the ovary/operculum rather than the straight hairs of E. benthamii. 
One collection from the Philippines, R.S. Williams $937(\mathrm{NY}(\times 2))$, is rather distinct from most of the material of this species in the Philippines in its acaulescent habit and sessile upper leaves. It also has large bracts. None of these characters is unique in the species but the combination is unusual. Further collections are necessary to see whether this is distinct from Epithema ceylanicum.

Some collections of this species have strongly variegated leaves but this character does not appear to have any taxonomic significance.

4. Epithema dolichopodum Hilliard \& B.L.Burtt, Edinburgh J. Bot. 54: 112 (1997). - TYPE: Malaysia, Sabah, Lahad Datu, Bukit Tempadong, 100-150 m, 11 June 1984, Beaman, J.H. 10068 (holotype E; isotypes GH, K, L, MSC, NY, US). (Fig. 6)

Herb 7-28 cm tall, acaulescent with first leaves arising at ground level, sometimes with 1-2 additional elongated internodes, indumentum of vegetative organs sparsely to densely strigose and/or setose, hairs to $0.6 \mathrm{~mm}$ long; stem 2-6 mm wide with 0-2 nodes, internodes to $8 \mathrm{~cm}$ long. Leaves membranous to strongly membranous, petiolate, solitary, opposite one or more peduncles; petioles (1.5-)5-37 cm; blades 5-24 × 4-15.5 cm, cordate, broadly ovate or occasionally sub-spherical, symmetrical to barely asymmetrical, apex usually rounded, occasionally acute, base cordate or sub-truncate, occasionally sub-auriculate, inserted evenly on petiole or not, margin sub-entire to dentate and bidentate. Inflorescences 1-5 per plant; peduncles (2-)9-27 cm long, originating from the base of the plant or opposite solitary leaves; bracts pale green or green, cucullate but not always enclosing entire inflorescence, 10-25 × 6-14 mm, 1.2-2.5 times as long as wide, margin dentate; pedicels 2.1-4.6 mm long, sub-glabrous to densely pubescent, hairs minute and hooked. Calyx lobes purple or purple only at tips, 3.8-5.6 mm long; tube $2-4 \times 1.5-3 \mathrm{~mm}$, lobes triangular, $1.3-2.7 \times$ 0.8-2 mm; minutely densely pubescent outside, hairs white; glabrous to very sparsely pubescent inside, hairs minute, in tips of lobes. Corolla tube white, lobes blue, with or without purple markings on lobes, 5-8.4 mm long; tube cylindrical, 3.3-6.3 × 1.3-3 $\mathrm{mm}$, lobes $1.7-2.1 \mathrm{~mm}$ long, margin slightly fimbriate to fimbriate; band of villous hair inside. Stamen filaments $1 \mathrm{~mm}$ long; anther characters unknown; staminodes $1 \mathrm{~mm}$ long. Nectary of two discrete lobes, 0.4-1.5 × 0.8-1.7 mm, margin undulate. Ovary sub-cylindrical to sub-spherical, $0.8-1.3 \times 0.6-1.3 \mathrm{~mm}$, minutely pubescent on upper portion of ovary, medium to high density, hairs hooked, hyaline or white, $0.04-0.1 \mathrm{~mm}$ long; style 3.7-4.1 mm long, glabrous or rarely with minute, hooked hairs; stigma c. $0.5 \mathrm{~mm}$ wide. Fruit cylindrical to obovate, $2.3-3 \times 2.1-2.5 \mathrm{~mm}$; operculum $0.6-0.8$ $\mathrm{mm}$ long, glabrous or as on ovary. Seed narrowly ovoid to ovoid, $0.3-0.6 \times 0.1-0.2$, dark brown, pattern partially spiralled or spiralled, regular.

Distribution. Malaysia (Sabah), Philippines (Palawan).

Habitat and ecology. Lithophytic on limestone. Found on boulders, outcrops, cave entrance walls in humid and shaded places at 90-150 m altitude. Flowering and fruiting in June, September-November. 


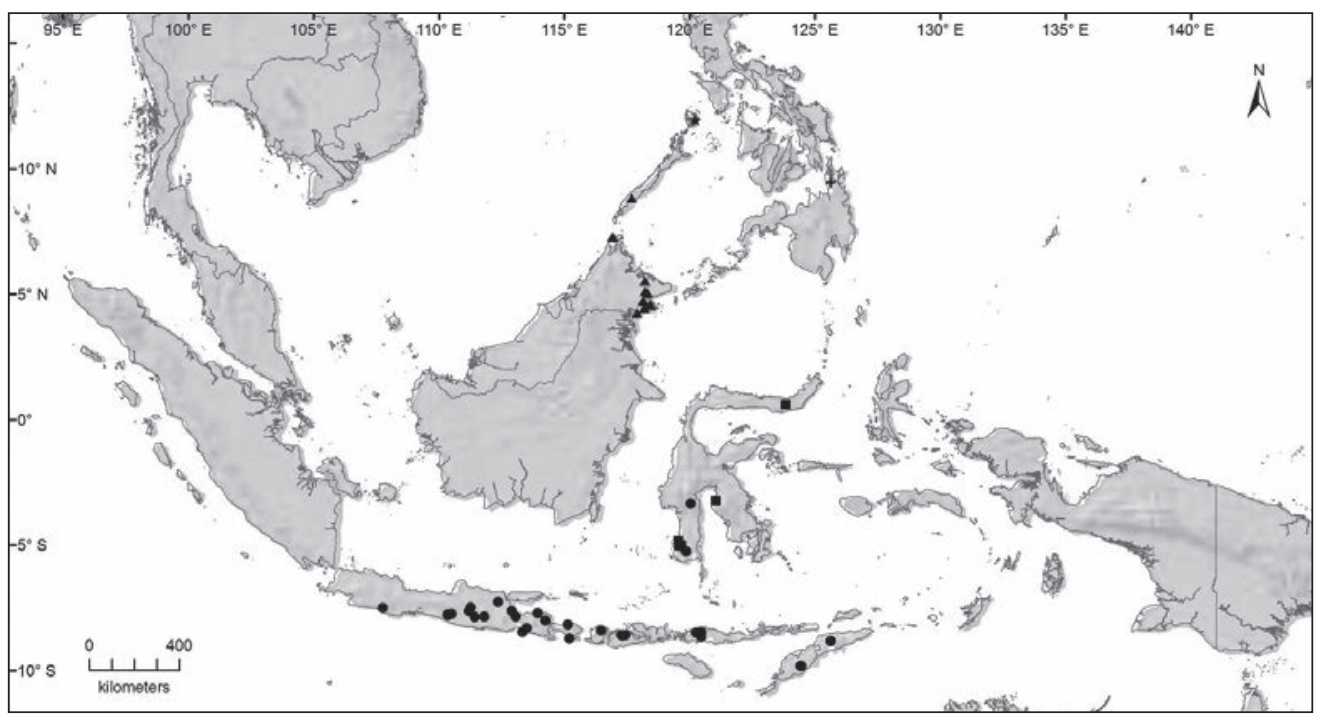

Fig. 6. Distribution of Epithema dolichopodum Hilliard \& B.L.Burtt ( $\mathbf{\Delta})$, Epithema horsfieldii (R.Br.) DC. (•), Epithema madulidii Hilliard \& B.L.Burtt ( $\star$ ), Epithema philippinum (Hilliard \& B.L.Burtt) Bransgrove (+) and Epithema tenerum (C.B.Clarke) Hilliard \& B.L.Burtt (ם).

Provisional IUCN conservation assessment. Least Concern (LC). Although this species is not especially widespread, its distribution is not restricted enough to fall into the Vulnerable category under the B criteria if there were accompanying threats. However, as it frequently occurs on karst limestone, which is often commercially exploited, its status should be monitored.

Additional specimens examined. MALAYSIA: Borneo: Sabah: Hutan Simpan Madai, 15 Sep 1976, Tamura, M. \& Hotta, M. 690 (KYO); Kudat, Pulau Balembangan, Oct 1994, Anthonysamy, S. et al. SB7 (E); Kudat, Pulau Balembangan, Kampung Sina, 9 Apr 1977, Stone, B.C. \& Anderson, E.F. SAN86719 (KLU); Kunak, Madai Hill, 9 Jun 1996, Lim, S.P. et al. LSP681 (SING); ibidem, 9 Jun 1996, Lim, S.P. et al. LSP675 (SING); Lahad Datu, Segama River, Batu Sarang, 25 Aug 1999, Kiew, R. RK4759 (SING); Madai Baturong Forest Reserve, Madai Caves, 14 Nov 1968, Kokawa, S. \& Hotta, M. 1125 (KYO, L); ibidem, 14 Nov 1968, Kokawa, S. \& Hotta, M. 1073 (KYO); Segarong, Kiew, R. et al. RK4321 (K, SING); ibidem, Symington, C.F. \& Agama, J. 9361 (K, SING); Sukau, Bod Tai, 14 Sep 1996, Kiew, R. \& Lim, S.P. RK4138 (K, SING); Tawao, Oct 1922-Mar 1923, Elmer, A.D.E. 20569 (G, GH, K, L, SING); Tawau District, Mount Wullersdorf Forest Reserve, Teck Guan Quarry, Nadiah, I. et al. SAN149136 (SING).

PHILIPPINES: Palawan: Mt Kabangaan, Apr 1929, Edaño, G. BS77711 (GH); Mt Mantalingajan, Apr 1929, Edaño, G. BS77570 (GH, SING).

Notes. This species is most easily confused with other acaulescent species such as Epithema strigosum, E. longipetiolatum and E. rennellense. One of the differentiating features of Epithema dolichopodum are the very long peduncles which are usually 
$(1-) 2(-3)$ times the height of the rest of the plant. In Epithema rennellense, E. longipetiolatum and E. strigosum the peduncles are shorter to barely longer than the height of the plant. Other differences are discussed in the notes section of the other species.

The hairs on the ovary and operculum are hooked and minute, the only variation being the density of the hairs. While it is infrequent, the lower style may be pubescent with hooked hairs. Fruit with glabrous and pubescent styles were observed from the same plant.

While the upper surface of the leaves can be densely strigose, the surface of the leaves and the stem may appear glabrous due to the small size of the hairs.

The collections available for examination are from relatively few sites, four of which were in close proximity to each other.

To-date, the only other species of Epithema found in Sabah are E. saxatile and E. sarawakense.

5. Epithema horsfieldii (R.Br.) A.DC., Prod. 9: 279 (1845). - Aikinia horsfieldii R.Br., Pl. Asiat. Rar. 3: 66 (1932). - TYPE: Indonesia, Java, 1802-1818, Horsfield, T. 85, also labelled Cyrtandr. 3 (lectotype BM [BM001125214], designated here; isolectotype K (×2)). (Fig. 6)

Epithema difformis Span., Linnaea 15: 331 (1841). - Carpocalymna monophylla Zipp. ex Span., Linnaea 15: 331 (1841), nom. inval. - TYPE: Indonesia, Timor, 1841, Spanoghe, J.B. 57 (lectotype P, designated here; isolectotype L).

Epithema horsfieldii var. epiphyllum Hilliard \& B.L.Burtt, Edinburgh J. Bot. 54: 112 (1997). - TYPE: Indonesia, Western Sumbawa, Batudulang and Sampar Olat Ranges, Mt Batulanteh, April 1961, Kostermans, A.J.G.H. 18839 (holotype L; isotypes BO (image seen), K).

Herb 3.2-22 cm high, caulescent (but can appear as just one large leaf with the stem mistaken for a petiole), unifoliate or rarely with two leaves; stem 0.5-3.8 mm wide. Leaves sessile (but see above), membranous to strongly membranous; blade 3.2-17.5 $\times 2.4-13 \mathrm{~cm}$, usually cordate or sub-cordate but also to oblong, symmetrical or not, apex broadly acute to rounded, base cordate to truncate, inserted evenly on petiole, margin entire to dentate, bidentate, serrate or crenate, arrangement of veins variable but the lower secondary veins often appearing to arise from around the base of the peduncles, often widely spaced, occasionally running down sides of midrib; upper surface pale green to green; lower surface light green or purplish. Inflorescences 1-15 per plant; peduncles $0.3-13.3 \mathrm{~cm}$ long, originating from the midrib at the base of the blade, or from the midrib up to $2.3 \mathrm{~cm}$ from the base; bracts cucullate, sub-cucullate or small and only enclosing a small proportion of the inflorescence, 2-22 × 3-12 mm, margin variable, irregularly dentate to dentate, occasionally lobed; pedicels $0.7-5 \mathrm{~mm}$ long. Calyx 2.5-5.7 mm long; tube 1-4.4 × 0.9-2.7 mm; lobes 0.6-5 × 0.4-2.3 mm; 
glabrous inside or rarely sub-glabrous with hairs to $0.1 \mathrm{~mm}$ long. Corolla blue, lilac, lilac-blue, violet-purple and white in proximal half of the two upper lobes or violet only at tips, 6.5-12 mm long; tube cylindrical 4-9 × 0.9-2 mm; lobes 1.5-4.2 × 1 $\mathrm{mm}$, margin undulate to slightly fimbriate; band of villous hair inside at one third to half way up the tube, occasionally the band is incomplete or the hair sparse. Stamens 0.7-1.9 mm long; filaments $0.6-1.7 \mathrm{~mm}$ long; anthers $0.3-0.6 \mathrm{~mm}$ long; staminodes 0.4-1 mm long. Nectary apparently absent or one or two lobes, sometimes encircling the ovary, margin entire or undulate and of variable height, $0.2-1.3 \times 0.1-1.5 \mathrm{~mm}$. Ovary sub-cylindrical to sub-spherical, $0.7-1.6 \times 0.5-1.7 \mathrm{~mm}$, sparsely to densely pubescent, hairs hooked to $0.1 \mathrm{~mm}$ long, on upper half of ovary or the uppermost portion of the ovary below the base of the style; style $0.5-5.8 \mathrm{~mm}$ long, glabrous to pubescent at the base, hairs hooked, 0.04-0.2 mm long; stigma 0.3-0.6 mm wide. Fruit obovate-cylindrical, shortly cylindrical or sub-spherical, 1.3-3 × 1-3 mm long; operculum $0.5-1 \mathrm{~mm}$ long, glabrous or pubescent in upper parts, hairs medium to high density, hooked, white, $0.04-0.13 \mathrm{~mm}$ long. Seed narrowly ovoid to ovoid, infrequently slightly sigmoid, ends acute or constricted, $0.3-0.6 \times 0.1-0.2 \mathrm{~mm}$, light to dark brown, pattern straight to spiralled and more or less regular.

Distribution. Indonesia (Java, Lesser Sunda Islands, Sulawesi), Timor Leste.

Habitat and ecology. Primarily lithophytic on limestone hills, rocks and cliffs, usually in shaded to heavily shaded areas. Also found in roadside cuttings. Collected at 40$900 \mathrm{~m}$ altitude. Flowering and fruiting January-May.

Provisional IUCN conservation assessment. Data Deficient (DD). Although this species is widespread and would normally be considered to be Least Concern there are surprisingly few recent collections from much of its range, particularly from Java. It is unclear whether this is due to a lack of collecting activity or whether it really has declined over much of its range. This will need to be investigated before a realistic conservation assessment can be made.

Additional specimens examined. INDONESIA: Bali: Singaraja, Gitgit Waterfall, 5 Apr 1936, van Steenis, C.G.G.J. 7788 (BO); Gunung Kelatakan, 2 Aug 1918, Maier Sarip, R. 162 (BO). Java: s.l., 8 May 1931, Clason, E.W. C99 (L); Zollinger, H. 2002 (S). Jawa Timur: Surabaya, Mantup, Coster, C. s.n. (BO); Gunung Wilis, 1914, Backer, C.A.B. 11469 (BO); Besuki, Gunung Ringit, Kampong Agoeng, 8 Mar 1940, Buwalda, P. 7542 (BO); Besuki, Jang Plateau, Djeloewang Ravine, 18 Jul 1938-19 Jul 1938, van Steenis, C.G.G.J. 11079 (BO); Besuki, Puger, 28 Feb 1940, Buwalda, P. 7225 (BO, L); Nusa Baron, 21 Feb 1845, Zollinger, H. 2612 (BO); Pasuruan, 18 Mar 1929, Backer, C.A.B. 36621 (L); Ponorogo to Soemoroto, Jan 1918, Beumée, J.G.B. 1328 (BO); Soekapoera, Gorge of Oemboelan, 12 Apr 1925, Jeswiet, J. 670 (WAG). Jawa Tengah: Gunung Lawoe, 1913, Backer, C.A.B. 6607 (L); ibidem, 1913, Backer, C.A.B. 6687 (BO); Yogyakarta, Gunung Kidoel, 1912, Backer, C.A.B. 2789 (BO, L); Yogyakarta, Unknown (ex Herb. Lugd. Batav.) s.n. (L); Yogyakarta, Prambanan, Feb 1912, Ridley, H.N. s.n. (K). Jawa Barat: Pameungpeuk, Goenoeng Karikil, Jan 1933, Jacobson, E. 199 (BO). Nusa Tenggara Barat: Lombok, Sambelia, Gunung Rinjani, 17 Feb 1998, de Wilde, 
W.J.J.O. \& de Wilde-Duyfjes, B.E.E. 21940 (E, L); ibidem, 2 May 1961, Kostermans, A.J.G.H. 18629 (K, L); Western Sumbawa, Batudulang, Mt Batulante, 10 Apr 1961, Kostermans, A.J.G.H. 18053B (K); Western Sumbawa, Olat seli, Pernek, 17 May 1961, Kuswata 213 (BO); Western Sumbawa, Sumbawa Besar, 29 Mar 2004, Hoover, W.S. et al. Deden151 (US); Western Sumbawa, Sumbawa Besar, Semongkat Atas, 26 Mar 2004, Hoover, W.S. et al. HW11417 (BO, US); ibidem, 1 May 1961, Kuswata 105 (BO, L); ibidem, 2 May 1961, Kuswata 121 (K, L). Nusa Tenggara Timur: Flores, Loeters, J.J. 1625 (L); Flores, Lempe to Waewako, 26 Mar 1974, Schmutz, E. 3623 (L); Flores, Waewako, 21 Feb 1978, Schmutz, E. 4027 (L); Western Flores, Manggarai, 18 Feb 1979, Schmutz, E. 4345 (L); ibidem, 6 Feb 1981, Schmutz, E. 4732 (L); Timor, Naukae, 29 Jan 1971, Kooy, C.W. 789 (L); Timor, Nikiniki, 20 Feb 1966, Kooy, C.W. 403 (L). Sulawesi Selatan: Bulukumba, Malino, 2 Apr 1922, Bünnemeijer, H.A.B. 10713 (BO, L); South ibidem, 8 Apr 1921, Bünnemeijer, H.A.B. 10890 (BO); Bulukumba, Malino, Bulutana, 10 Feb 2000, Mendum, M. et al. 0034 (E, SING); Maros, 23 Jan 1998, de Wilde, W.J.J.O. \& de Wilde-Duyfjes, B.E.E. 21918 (E, L).

Notes. Hilliard \& Burtt(1997) described the variety Epithema horsfieldii var. epiphyllum which they distinguished from the type variety by the position of the inflorescences. In Epithema horsfieldii var. horsfieldii they usually arise from the base of the leaf blade and in E. horsfieldii var. epiphyllum from the midrib, up to $2.3 \mathrm{~cm}$ from the base of the blade. Occasionally the inflorescences of the plants placed in Epithema horsfieldii var. horsfieldii also arise from the lower midrib but these specimens are more densely villous inside the corolla than the material placed in E. horsfieldii var. epiphyllum. In addition the material assigned to Epithema horsfieldii var. horsfieldii is generally more pubescent than E. horsfieldii var. epiphyllum and has larger and more cucullate bracts. However, specimens with the morphology of the type variety were found disjunctly in Java and Timor and that of Epithema horsfieldii var. epiphyllum in Lombok, Sumbawa and Flores. This would be biogeographically unlikely. The material from Sulawesi is rather different again as much of the material has inflorescences arising in a slight groove at the base of the leaf blade which is not found in the two existing varieties whereas other specimens are more similar to the type variety. What is clear is that the patterns of variation require considerably more study and that if varieties are to be recognised they should only be so after more collecting from throughout the range of the species (but particularly in Timor) and after a more thorough understanding of the variation.

6. Epithema involucratum (Roxb.) B.L.Burtt, Notes Roy. Bot. Gard. Edinburgh 22: 308 (1958). - Gratiola involucrata Roxb., [Hort. Bengal. 80 (1810), nom. nud.] Fl. Indica ed. 1, 138 (1820). - Epithema roxburghii A.DC., Prod. 9: 279. (1845), nom. superfl. - TYPE: "Moluccas", Roxburgh s.n. (lectotype PDA, designated here). (Fig. 7)

Aikinia brunonis Wall., Pl. Asiat. Rar. 3: 66 (1832); G.Don, Gen. Hist. 4: 665 (1838). Epithema brunonis (Wall.) Decne., Nouv. Ann. Mus. Hist. Nat. 3: 404 (1834); Decne., Herbarii Timorensis Descriptio 76 (1835); A.DC., Prodr. 9: 279 (1845); Clarke in 


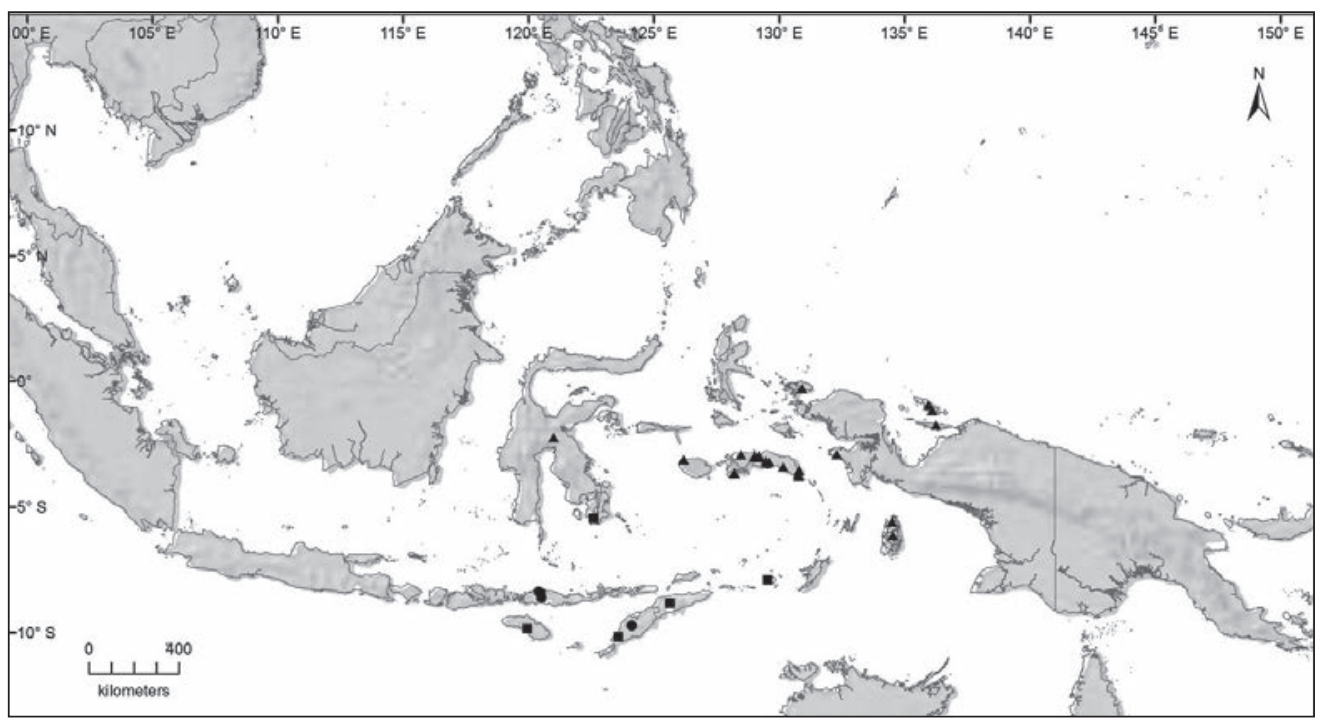

Fig. 7. Distribution of Epithema involucratum (Roxb.) B.L.Burtt (घ), Epithema longipetiolatum (Merr.) Hilliard \& B.L.Burtt ( $\boldsymbol{\Delta})$, Epithema longitubum Hilliard \& B.L.Burtt $(\bullet)$.

A.DC. \& C.DC., Monogr. Phan. 5(1): 179 (1883). - TYPE: Indonesia, East Nusa Tenggara, Timor, Coepang, Apr 1803, Brown, R. s.n. (lectotype BM, designated here).

Epithema brunonis var. violaceum C.B.Clarke in A.DC. \& C.DC., Monogr. Phan. 5(1): 179 (1883). - Epithema violaceum Span., Linnaea 15: 330 (1841), nom. nud. - TYPE: Timor Leste, Spanoghe 58 (holotype P; isotype L).

Herb 4.5-20 cm high, caulescent, indumentum strigose to hispid, hairs sparse to medium density, to $0.3 \mathrm{~mm}$ long on the stem, petioles, peduncles, lower bract, and the outside of the calyx, to $0.8 \mathrm{~mm}$ long on the upper and lower surface of the leaves, indumentum of lower bract, pedicels and the outside of the calyx also of minute hairs; stem 1-3 mm wide with 2 to 3 nodes, internodes $1.3-3 \mathrm{~cm}$ long. Leaves membranous and petiolate, upper leaves opposite; petioles $0.2-4.5 \mathrm{~cm}$ long; blades $1.3-6 \times 1-5.4$ $\mathrm{cm}$, ovate or cordate, rarely almost oblong symmetrical or barely asymmetrical, apex rounded to truncate, more rarely broadly acute, base cordate or truncate, inserted evenly on petiole or not, margin entire or with few, minute teeth, undulate. Inflorescences up to 5 per plant, terminal, in lower leaf axils or on base of petioles; peduncles 1.3-5.5 cm long; bracts cucullate but not always enclosing entire inflorescence, $7-17 \times 4-10$ $\mathrm{mm}$, margin variable, almost entire to dentate, upper surface sub-glabrous, hairs to 0.2 $\mathrm{mm}$ long, placed primarily towards margins of the bract; pedicels $0.9-3.5 \mathrm{~mm}$ long, indumentum may include strigose hairs to $0.5 \mathrm{~mm}$ long. Calyx 2.9-4.4 mm long, tube 1.7-2.5 × 1-2.5 mm, lobes 1-2.1 × 0.6-1.9 mm; glabrous inside. Corolla light blue or bluish to dark violet, tube may be white, 9-11 mm long; tube 5-6 × 1.5-2 mm; lobes $4-5 \times 3-4 \mathrm{~mm}$, almost as long as the tube; glabrous outside and inside. Stamen 
filaments $1.2 \mathrm{~mm}$ long; anther dimensions unknown; staminodes $0.7 \mathrm{~mm}$ long. Nectary of two discrete lobes, $0.6 \times 0.6 \mathrm{~mm}$. Ovary cylindrical or sub-cylindrical, $0.8-1 \times 0.7$ $\mathrm{mm}$, pubescent, hairs dense, hooked, white, to $0.1 \mathrm{~mm}$ long on upper portion of ovary; style $3.5 \mathrm{~mm}$ long, glabrous or sub-glabrous, hairs, when present, hooked and on lower style; stigma to $0.5 \mathrm{~mm}$ wide. Fruit sub-cylindrical to sub-spherical, 1.7-1.9 $\times 1.7$ $\mathrm{mm}$; operculum $0.6-0.8 \mathrm{~mm}$ long, sparsely to densely pubescent, hairs hooked, to 0.1 $\mathrm{mm}$ long, on entire operculum. Seed fusiform to broadly oval, $0.3-0.5 \times 0.1-0.2 \mathrm{~mm}$; pattern straight to spiralled and even.

Distribution. Indonesia (Sulawesi, Timor, Maluku), Timor Leste.

Habitat and ecology. In shaded areas. Highly likely to be lithophytic on limestone and found in humid places and/or near water.

Provisional IUCN conservation assessment. Data Deficient (DD). The known localities of this species suggest a fairly widespread distribution. However, it has not been collected often, many of the collections are fairly old and the exact localities of some of these older specimens are not known. We, therefore, assign it the status of Data Deficient until more information becomes available.

Additional specimens examined. INDONESIA: Maluku: Babar Islands, Pulau Weten, 28 Feb 1956, van Borssum Waalkes, J. 3025 (BO, L). Nusa Tenggara Timur: Sumba, Laora, 10 Nov 1925, Iboet 221 (BO); Timor, Spanoghe, J.B. 226 (L); ibidem, Unknown (probably Spanoghe) 226 (L). Sulawesi Tenggarah: Pulau Butung, Baoe Baoe, 20 Feb 1929, Kjellberg, G.K. 314 (BO, S); ibidem, 20 Feb 1929, Kjellberg, G.K. 315 (S).

Notes.The key macroscopic features distinguishing Epithema involucratum from other species are the cordate to ovate leaves with clearly rounded leaf tips and that it is one of the few species that is glabrous inside the corolla. Epithema longitubum and some specimens of E. ceylanicum are similar to E. involucratum. The Epithema ceylanicum specimens can be distinguished from E. involucratum by the presence of an indumentum inside the corolla, smaller inflorescences and shorter corolla length. Epithema longitubum can be distinguished by the often longer corolla length and presence of an indumentum in the corolla. While there were not many corollas available to examine, it would seem that the lobes of E. involucratum are quite large in comparison to other species.

Van Borssum Waalkes 3025 (L) from Maluku is rather intermediate between Epithema involucratum and E. longitubum. It is included in Epithema involucratum on the basis of its leaf shape, absence of hair in the corolla and the lack of an upper, solitary leaf. It does have medium to high density, strigose hairs on the inner side of the calyx lobes which is unusual for either species. Its inclusion in Epithema involucratum significantly extends the known distribution of E. involucratum, as it would also have done for E. longitubum if it were included there.

Kjellberg 314(S) from Sulawesi also extends the range of Epithema involucratum although, while most of its characters are typical for E. involucratum, it has hooked hairs at medium density on the ovary which is unusual for this species. 
Epithema brunonis var. violaceum is included in synonymy of E. involucratum. Clarke (1883) used depth of flower colour, length of the corolla and the leaf pubescence to delineate it from the type variety. The flower colour and leaf pubescence is not different from that seen in other E. involucratum specimens and the corolla length, from material that Clarke himself examined, is $9 \mathrm{~mm}$ not $12 \mathrm{~mm}$ as Clarke stated when differentiating the variety. The key differences are nominal; the shape of the base of a couple of leaves differ slightly and the peduncles can be up to $15 \mathrm{~mm}$ longer than in typical E. involucratum. Epithema brunonis var. violaceum, however, is within the range of variation for E. involucratum.

In the protologue of Aikinia brunonis there is no indication of a collector of the specimen used to make the description, only that it is based on a collection from Coepang (now called Kupang) in Timor which was flowering and fruiting in April 1803. There is a Robert Brown collection from Coepang collected in April 1803 in the BM. This specimen is lectotypified here.

7. Epithema longipetiolatum (Merr.) Hilliard \& B.L.Burtt, Edinburgh J. Bot. 54: 112 (1997). - Epithema brunonis var. longipetiolatum Merr., Philipp. Journ. Sci., C. 11: 313 (1916). - TYPE: Indonesia, Maluku, Ambon, Halong, 50-100 m, 26 September 1913, Robinson, C.B. 1727 (lectotype L, designated here; isolectotypes BO, K, US). (Fig. 7)

Herb 5-30 cm high, acaulescent or caulescent; stem 2-3 mm wide with two nodes, internodes 7-10 cm long. Leaves membranous, petiolate, usually solitary but upper leaves may be opposite, opposite leaves are occasionally unequal in size; petioles ( 0 ) $0.5-12(-18) \mathrm{cm}$; blade 3-13.6 × 2.4-10.2 cm, cordate to broadly ovate or occasionally sub-lanceolate, symmetrical or asymmetrical, apex acute, base sub-cordate or subauriculate to cuneate, inserted evenly on petiole or distinctly offset, margin almost entire to serrulate or denticulate, veins often widely spaced; upper surface glabrous or sparsely to densely strigose, hairs to $0.4 \mathrm{~mm}$ long, with or without a minute hooked pubescence; lower surface finely pubescent, at medium to high density, surface often appearing fuzzy. Inflorescences 1-6 per plant; peduncles $0.2-6 \mathrm{~cm}$ long, terminal or in the axils of upper leaves; bracts cucullate but not enclosing the entire inflorescence, 5-12 × 4-9 mm, margin somewhat dentate to dentate and serrate, upper surface sub-glabrous to sparsely strigose, hairs straight, to $0.2 \mathrm{~mm}$ long, denser towards the margin; pedicels 1-4.2 mm long. Calyx 2.7-5.6 mm long; tube 1.5-5 × 1.5-3.8 mm, lobes $1.2-2.7 \times 0.6-1.7 \mathrm{~mm}$; minutely pubescent (often finely) outside, occasionally strigose, at medium to high density, all hairs hooked or primarily hooked and to 0.25 $\mathrm{mm}$ long, hairs on inside of lobe tips to $0.13 \mathrm{~mm}$, occasionally sub-glabrous. Corolla tube white, lobes lilac or pale lilac, lobes with darker coloured but variable markings, 5.5-10.2 mm long; tube cylindrical, 4.2-6.4 × 1.5-1.9 mm, lobes 1.2-3.8 mm long; with a band of villous hairs inside around middle, up to $1 \mathrm{~mm}$ wide. Stamens 2.1-3.1 $\mathrm{mm}$ long; filaments $1.5-2.3 \mathrm{~mm}$ long; anthers $0.6-0.8 \mathrm{~mm}$ long; staminodes $1.2-2.3$ $\mathrm{mm}$ long. Nectary of two discrete lobes, margin entire or undulate, $0.5-1.1 \times 0.7-1.7$ 
mm. Ovary sub-cylindrical or sub-spherical, 1-1.5 $\times 0.7-1.3 \mathrm{~mm}$, densely pilose, hairs usually straight or occasionally straight and hooked, placed on upper portion of ovary, 0.04-0.08(-0.25) mm long; style 3.1-4.4 mm long, wider at base to $0.33 \mathrm{~mm}$, style glabrous or with very few hairs at base, these straight, to $0.17 \mathrm{~mm}$ long; stigma $0.4 \mathrm{~mm}$ wide. Fruit obovate to sub-cylindrical, 2-4 × 2-3.5 mm; operculum 0.6-1.9 $\mathrm{mm}$, finely setose or pilose, hairs medium to high density, as on ovary. Seed narrowly to broadly ovoid, $0.3-0.5 \times 0.1-0.2 \mathrm{~mm}$, light to medium brown, pattern straight to partially spiralled, often with thick, clearly defined walls.

Distribution. Indonesia (Ambon, Buru, Seram, Aru, Sulawesi, Papua).

Habitat and ecology. Lithophytic on limestone. In humid, shaded places, often on rocks in stream beds at 0-1770 m altitude. Flowering and fruiting throughout the year.

Provisional IUCN conservation assessment. Least Concern (LC). This species is widespread. However, as it frequently occurs on karst limestone, which is often commercially exploited, its status should be monitored.

Additional specimens examined. INDONESIA: Maluku: Aru Islands, Pulau Kobroor, Namadoeboele, 10 Jun 1938, Buwalda, P. 5202 (BO); Aru Islands, Pulau Wokam, Dosinamalaoe, 1 Jun 1938, Buwalda, P. 5110 (A, BO, K, L); Buru, NW Buru, Bara, 5 Dec 1984, van Balgooy, M.M.J. 5066 (BO, L); Buru, NW Buru, Bara, Waeduna River, Nooteboom, H.P. 5217 (L); ibidem, 25 Nov 1984, van Balgooy, M.M.J. 4889 (E, L); Ambon, Boerlage, J.G. 528 (BO); ibidem, Teijsmann, J.E. s.n. (BO); Seram, 9 Jan 1938, Eyma, P.J. 2574 (BO, L); Seram, Kampong Kwaos, Gunung Toenlean, 8 Sep 1938, Buwalda, P. 6006 (K, L); Seram, Kampong Selagor, Gunung Selagor, 26 Aug 1938, Buwalda, P. 5748 (BO, L); Seram, Kecamatan Seram Utara, Goa Pohon Damar to Gunung Roihelu, 24 Jan 1985, Kato, M. et al. C.5232 (L); Seram, Kecamatan Seram Utara, Goa Pohon Damar to Sawai, 27 Jan 1985, Kato, M. et al. C.5728 (L); Seram, Pileana, 28 Oct 1937, Eyma, P.J. 1826 (A, BO, K, L, SING); Seram, 4 Feb 1918, Kornassi 967 (BO, L); Seram, Manusela National Park, Gunung Binaia, 26 Aug 1987, Argent, G.C.G. C8754a (E); Seram, Tehoru, Hatumete Pass, 29 Nov 1917, Kornassi 621 (BO, L); Seram, Tehoru, Saunulu, Murkele Ridge, 21 Jul 1986, Kato, M. et al. C.11441 (L); ibidem, 21 Feb 1985, Kato, M. et al. C.5144 (L); Seram, Taniwel, Between Buria and Wae Mala, 3 Feb 1985, Kato, M. et al. C.6007 (A, L). Papua Barat: Kabupaten Raja Ampat, Waigeo Island, Tuluk Mayalipit, Warsamdin, 1 May 2008, Okada, H. \& Tsukaya, H. OT-48 (BO); Biak, Aet \& Idjan 942 (BO, L); Biak, Gunung Wawah, 22 Jul 1939, Aet \& Idjan 32 (A, BO, K, L); FakFak, Fak-Fak River, 23 Feb 1962, Vink, W. BW12141 (L). Sulawesi Tengah: Soroako, Mt. Wawonseru, 2 Jul 1979, Hennipman, E. 610g (A, E, L).

Notes. The specimens of Epithema longipetiolatum with leaves arising from ground level could be confused with E. rennellense and E. strigosum although there is no overlap in distribution. Leaves arising from ground level are always present in $E$. rennellense and E. strigosum and opposite leaves are never seen in these species. The leaf margin of Epithema strigosum has large wide teeth (entire to weakly dentate in $E$. longipetiolatum). Epithema rennellense has straight and hooked hairs on the ovary and operculum (usually only straight in E. longipetiolatum). 
'Longipetiolatum' refers as much to the length of the secondary internode as to the petioles, giving the plant an elongate appearance. For example, in Kornassi 967 (L), the secondary internodes are a third of the total plant height and equal to the primary internode and the upper petiole and blade combined.

Epithema longipetiolatum may have solitary leaves only, opposite leaves only, or opposite and solitary leaves. This does not correlate with geography and both types can be found within a population. For example, duplicates of the type collection have only opposite leaves or only solitary leaves while Argent C8754a (E) from Seram includes plants that have only opposite leaves or both opposite and solitary leaves.

Some specimens of Epithema longipetiolatum are similar to specimens of $E$. benthamii. For example, one duplicate of Eyma 1826 (L), from Seram, has a similar leaf shape and margin to E. benthamii. We have seen no specimens of Epithema benthamii from Seram, but E. longipetiolatum and E. benthamii are found in the same locality in the Waeduna River district on Buru Island. The ovary and operculum hair of specimens of Epithema longipetiolatum found on Buru Island often have a mixture of long (to $0.25 \mathrm{~mm}$ ), almost villous hairs that are straight or straight and hooked like E. benthamii, in addition to the expected, short hairs. This contrasts with the type specimen of Epithema longipetiolatum from Ambon, the E. longipetiolatum specimens from Seram, Wokam Island and the Aet \& Idjan 942 (L) specimen from Biak in West Papua, which have minute, pilose, straight hairs (rarely with a few hooked hairs) on the ovary and operculum. It is possible some hybridisation occurs between Epithema longipetiolatum and E. benthamii in these localities.

The minute hooked pubescence on the pedicels and outer calyx is quite distinctive, especially for the specimens from Seram. For most specimens of Epithema longipetiolatum, the indumentum of the upper leaf surface is of long, strigose hairs and looks somewhat like the indumentum of the upper surface of the leaves of E. benthamii. Specimens from Buru, however, have a dense, uniform and short indumentum, giving the leaves a fuzzy look.

8. Epithema longitubum Hilliard \& B.L.Burtt, Edinburgh J. Bot. 54: 112 (1997). TYPE: Timor, Nasinutan, 11 March 1939, Bloembergen, S. 3458 (holotype L; isotype BO (image seen)). (Fig. 7)

Herb 7.5-20 cm high, with a distinct (but sometimes short) stem before first leaf; stem 0.6-2.5 mm wide with two nodes, internodes 1.5-7 cm long. Leaves membranous, petiolate, upper leaves opposite or with an uppermost leaf solitary opposite a peduncle; petiole of lowest leaf 4-6 cm long, petioles of upper leaves $0.5-5.5 \mathrm{~cm}$; blade 1.7-11 $\times 1-7.8 \mathrm{~cm}$, sub-orbicular to broadly ovate or somewhat oblong, symmetrical or not, if not one side up to 1.3 times wider than the other, apex rounded to acute, base shallowly auriculate or cordate to rounded, inserted evenly on the petiole or not, margin almost entire to serrulate or denticulate, venation sub-opposite towards base, usually alternate towards leaf tip, widely spaced; upper surface with a longer indumentum of hairs to $1 \mathrm{~mm}$ long, with or without an additional shorter pubescence of hooked hairs; lower 
surface drying pale, indumentum as upper surface but less dense. Inflorescences 2-10 per plant; peduncles $0.5-8 \mathrm{~cm}$ long, terminal or in leaf axils; bracts diamond-shaped or trullate, cucullate but only enclosing a small portion of the inflorescence, $2-9 \times$ 3-6 mm, margin roughly entire with occasional dentation or with some crenate and dentate lobes, indumentum of lower surface variable with hairs to $0.5 \mathrm{~mm}$ long; upper surface sub-glabrous, sparsely and minutely pubescent or sparsely strigose; pedicels 0.75-4.2 mm long. Calyx 2.9-5.2(-7) mm long; tube 1.4-4.2 × 1.3-2.5 mm; lobes $1-2.9 \times 0.4-1.3 \mathrm{~mm}$; minutely and sparsely to densely pubescent outside and with or without larger hairs to $0.7 \mathrm{~mm}$ long at medium to high density throughout calyx or largely on lobes; glabrous to sparsely and minutely pubescent inside on upper part of lobes. Corolla blue-purple, c.10-17 mm long; tube cylindrical, 8-12 × 1.3-2 mm, up to 7 times as long as wide; lobes $2-5 \mathrm{~mm}$ long; with band of villous hair inside in lower part. Stamens 1.8-2.3 mm long; filaments $1-1.5 \mathrm{~mm}$ long; anthers $0.8 \mathrm{~mm}$ long; staminodes $0.6-1.4 \mathrm{~mm}$ long. Nectary of one or two lobes, partly to entirely encircling ovary, 0.5-0.9 × 1.3-1.5 mm, margin undulate and of varying height. Ovary sub-cylindrical or broadly ovoid with a truncate base, 0.9-1.1 $\times 0.4-0.6 \mathrm{~mm}$, densely pubescent on upper portion, hairs hooked; style 8.8-10 mm long, glabrous, rarely with few hooked hairs at base. Fruit sub-spherical to cylindrical, 1.3-2.5 × 1.7-2 $\mathrm{mm}$; operculum $0.5-0.6 \mathrm{~mm}$, pubescent throughout but denser at top, hairs hooked, to $0.08 \mathrm{~mm}$ long. Seed ovoid to broadly ovoid, $0.3-0.5 \times 0.1-0.2 \mathrm{~mm}$, pattern partially spiralled to spiralled, regular.

Distribution. Indonesia (Timor, Flores).

Habitat and ecology. Lithophytic on limestone in humid, shady places near or beside streams and cave entrances at 900-1200 m altitude. Flowering and fruiting March and May.

Provisional IUCN conservation assessment. Data Deficient (DD). The most recent collection of this species is from 1974 and the collection density on both Flores and Timor is too low to have an accurate idea of the current distribution.

Additional specimens examined. INDONESIA: Nusa Tenggara Timur: Flores, 25 Mar 1972, Verheijen, J.A.J. 3070 (L); ibidem, Mar 1974, Verheijen, J.A.J. 3323 (L); Flores, Ruteng-Mano, 16 May 1973, Schmutz, E.3208 (L); Flores, Ruteng-Reo, 7 Mar 1968, Schmutz, E.2127 (L); Timor, 30 Jan 1929, Walsh, M.E. 62 (BO); Timor, Nasinutan, 17 Mar 1939, Bloembergen, S. $3496(\mathrm{BO}, \mathrm{L})$.

Notes. This species is most similar to Epithema involucratum but the inflorescences, bract and corollas are usually much larger. Unfortunately the locality information on specimens of both Epithema longitubum and E. involucratum is poor and, therefore, it is as yet unclear whether these two species co-occur or not without further field work.

Although nectary characters have not proven useful in Epithema, and relatively few specimens have been examined for this species, it is interesting to note that the 
specimens from Flores have two nectary lobes and the specimen from Timor has one. The sampling density, however, is currently too low to make any firm conclusions.

9. Epithema madulidii Hilliard \& B.L.Burtt, Edinburgh J. Bot. 54: 112 (1997). TYPE: Philippines, Coron Island, Banol, 30 Sep 1993, Madulid, D.A., Agoo, Reynoso \& Fuentes, R. 11563 (holotype PNH; isotype E). (Fig. 6)

Herb 13.2-50 cm high, caulescent; stem 1-4.2 mm wide with two nodes, internodes 1.5-11 cm long. Leaves membranous or strongly membranous, lower leaf petiolate, upper leaves sessile; lower petioles 1-10 cm long, sparsely to densely hairy, often with two distinct lengths of hair, hairs white, to $1.1 \mathrm{~mm}$ long; lower leaf blade 9-13 $\times$ $4.2-7.2 \mathrm{~cm}$, upper leaves $2-4.5 \times 1.7-3.5 \mathrm{~cm}$, lower leaf cordate to ovate, base cordate to rounded, upper leaves ovate to elliptic, all leaves usually asymmetrical, one side 1.1-1.8 times wider than the other, apex broadly acute to rarely rounded, base cordate to obtuse, margin sub-entire to sinuate to minutely dentate, inserted evenly on petiole or not; upper surface sparsely to densely hairy, often with two distinct lengths of hair, hairs to $0.4 \mathrm{~mm}$ long; lower surface similar, hairs to $0.5 \mathrm{~mm}$ long, indumentum on veins often white and to $0.8 \mathrm{~mm}$ long. Inflorescences $1-3$ per plant, terminal; peduncles 1.4-8 cm long; bracts whitish at base, upper half violet, cucullate, partially enclosing inflorescence, $10-25 \times 6-15 \mathrm{~mm}$, margin dentate to irregularly dentate, lower surface sub-glabrous or sparsely strigose, upper surface glabrous; pedicels 1-4.2 mm long. Calyx 6.3-7 mm long; tube 3-5.2 × 2.1-3.8 mm, often drying a lighter colour than the lobes; lobes 1.5-2.9 × 1.1-3.8 mm, outside glabrous or with sparse hairs primarily on lobes, hairs to $0.6 \mathrm{~mm}$ long; sub-glabrous or pubescent inside, hairs straight, to $0.1 \mathrm{~mm}$ long, mostly only in lobes, occasionally in the tube. Corolla violet at tips, 5-10.4 mm long; tube cylindrical, 3.3-6.5 × 1.5-1.8 mm; lobes 1.7-4.5 mm long; sub-glabrous to pubescent on lobes outside, hairs hooked or straight, glabrous inside. Stamens 1.3-1.5 $\mathrm{mm}$ long; filaments $0.6-0.7 \mathrm{~mm}$ long; anthers $0.7-0.8 \mathrm{~mm}$ long; staminodes $0.6-0.75$ $\mathrm{mm}$. Nectary of one lobe, encircling half to two-thirds of the ovary, $0.5-0.9 \mathrm{~mm}$ high, margin entire or almost entire. Ovary sub-spherical, $0.7-1.1 \times 0.5-0.8 \mathrm{~mm}$, densely pubescent on upper portion of ovary, hairs hooked, white, to $0.04 \mathrm{~mm}$ long; style 6.3 mm long, glabrous or occasionally with few straight hairs. Fruit obovate to obovatecylindrical, 2.6-4 × 2-2.7 mm; operculum 0.6-0.9 mm, sub-glabrous to densely pubescent, hairs straight and hooked, to $0.13 \mathrm{~mm}$ long. Seed ovoid or with one side flattened, $0.5-0.6 \times 0.1-0.2 \mathrm{~mm}$, medium to dark brown, pattern more or less straight to spiralled, regular, lines of pattern thickened.

Distribution. Philippines (Coron Island).

Habitat and ecology. Found in shallow soil in limestone rock crevices in humid, shaded areas at c. 50-550 m.

Provisional IUCN conservation assessment. Data Deficient (DD). This species is only known from two collections from Coron Island and has both an EOO and AOO that 
would put it in the Critically Endangered category. However, this limestone island and neighbouring islands are not well collected and the impact of tourism on the habitat is not clear.

Additional specimen examined. PHILIPPINES: Palawan: Coron Island, Sep 1922, Lopez, G. BS41343 (BO, K, L, P, US).

Notes. This species is known from two collections from Coron Island collected in 1922 and 1993. Due to the poor condition of the specimens, particularly the Lopez BS41343 collection, some information such as leaf blade, base and tip shape is assessed from very few leaves.

The calyx of this species is quite large and the tube dries a very light brown colour in comparison to the lobes although this is based on a very small sample. The corolla tube is glabrous, or appears so, and, in combination with the light colour and large size of the calyx, is useful for identification from dried specimens. It is similar to the sessile-leaved form of Epithema saxatile but can be distinguished by the complete lack of hair inside the corolla and the presence of straight hairs on the operculum. In addition the calyx is larger (6.3-7 mm versus 3.3-5 mm) and there are hairs on the inside of the calyx lobes (lacking in Epithema saxatile).

10. Epithema membranaceum (King) Kiew, Malayan Naturalist J. 38(3): 36 (1985); B.L.Burtt, Thai For. Bull. (Bot.) 29: 93 (2001). - Argostemma membranaceum King, J. Asiat. Soc. Bengal, Pt. 2, 72: 145 (1903); Ridley, Fl. Malay Penins. 2: 24 (1923). - TYPE: Malaysia, Penang, Muka Head, Curtis 955 (lectotype SING, designated by Kiew (1985)). (Fig. 8, 9)

Herb 10-30 cm high, caulescent, indumentum of minute hooked pubescence and strigose hairs, usually sub-glabrous or sparse; stem $1.5 \mathrm{~mm}$ wide, can be red-brown in colour, with 2-3 nodes, internodes $0.6-12 \mathrm{~cm}$ long. Leaves often finely membranous, petiolate, upper leaves opposite, infrequently upper leaves solitary or sub-opposite; petioles $0.8-14.5 \mathrm{~cm}$ long; blade 3.6-11.5 × 3-10.5 cm, broadly ovate or cordate, occasionally sub-orbicular or almost oblong, symmetrical or barely asymmetrical, apex acute to rounded, upper portion of leaf sometimes curved, base sub-auriculate to truncate, inserted evenly on petiole, margin entire to serrate, crenate or with large, rounded dentation; upper surface glabrous to sparsely strigose (occasionally to medium density), hairs to $0.6 \mathrm{~mm}$ long; lower surface glabrous, sub-glabrous or covered in dense, minute hooked pubescence, giving a fuzzy appearance. Inflorescences (1-)3-9 per plant; peduncles 0.5-17 cm long, primarily from terminal leaf axils, rarely from the base of the petioles; bracts usually small, obovate, ovate or elliptic, not enclosing inflorescence, if sub-cucullate then only enclosing a small proportion of the inflorescence, 2-11 $\times 3-12 \mathrm{~mm}$, margin entire or with limited dentation, lower surface sub-glabrous, upper surface glabrous or with sparse hair towards bract margin; pedicels 1-3.5 mm long, densely and minutely pubescent, with or without few additional straight, strigose hairs to $0.3 \mathrm{~mm}$ long. Calyx 2.1-5.2 mm long; tube 


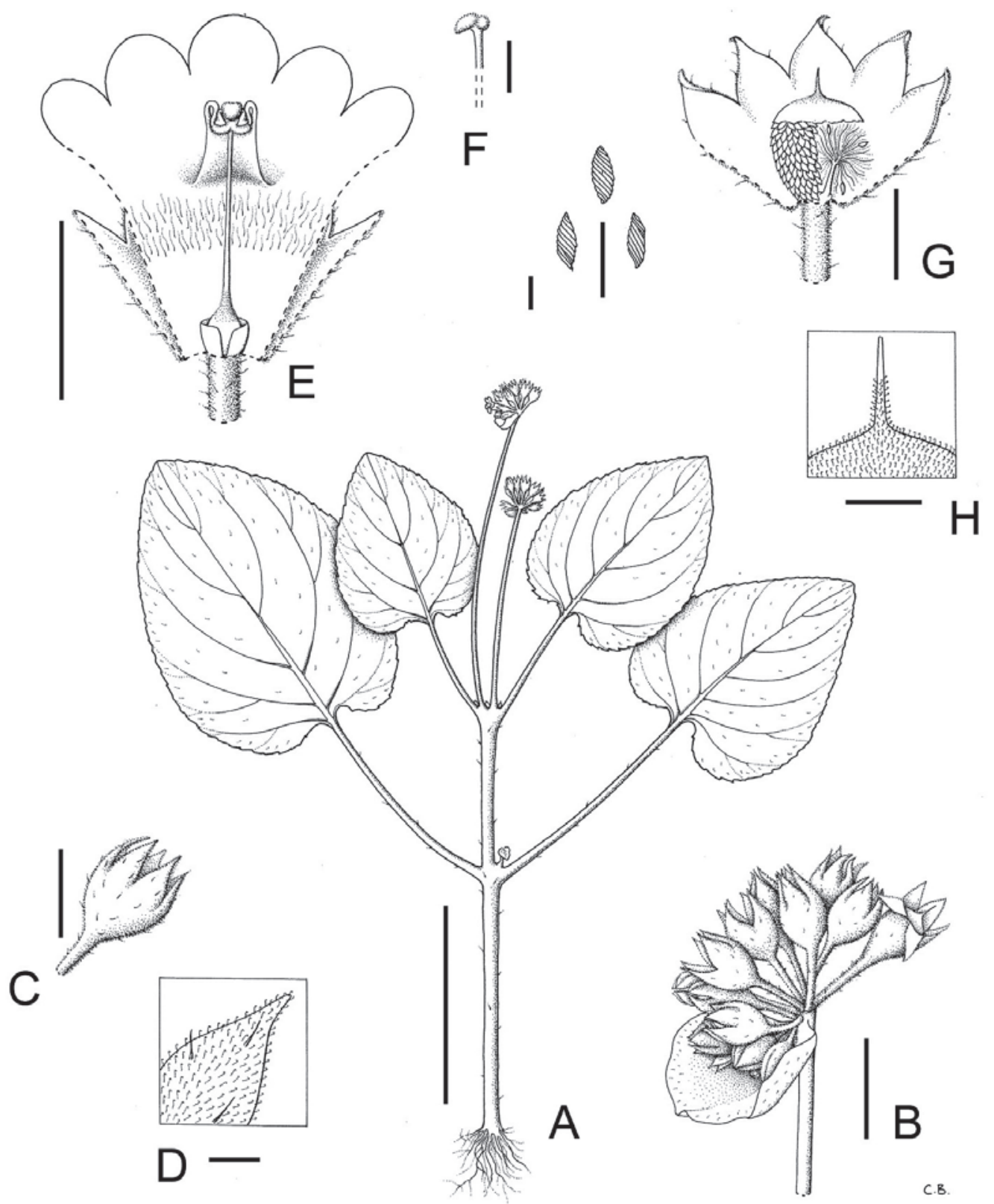

Fig. 8. Epithema membranaceum (King) Kiew A. Habit. B. Inflorescence. C. Calyx. D. Calyx indumentum detail. E. Flower opened out. F. Stigma lateral view. G. Fruit showing seeds, placenta and operculum. H. Operculum indumentum. I. Seeds. Scale bars: A = 4 $\mathrm{cm}, \mathrm{B}=5 \mathrm{~mm}, \mathrm{C}=3 \mathrm{~mm}, \mathrm{D}, \mathrm{H}, \mathrm{I}=0.5 \mathrm{~mm}, \mathrm{E}=4 \mathrm{~mm}, \mathrm{~F}=1 \mathrm{~mm}, \mathrm{G}=2 \mathrm{~mm}$. Drawn by Claire Banks from Larsen et al 42245 (A-D, G-I) and Larsen et al. 42312 (E, F). 


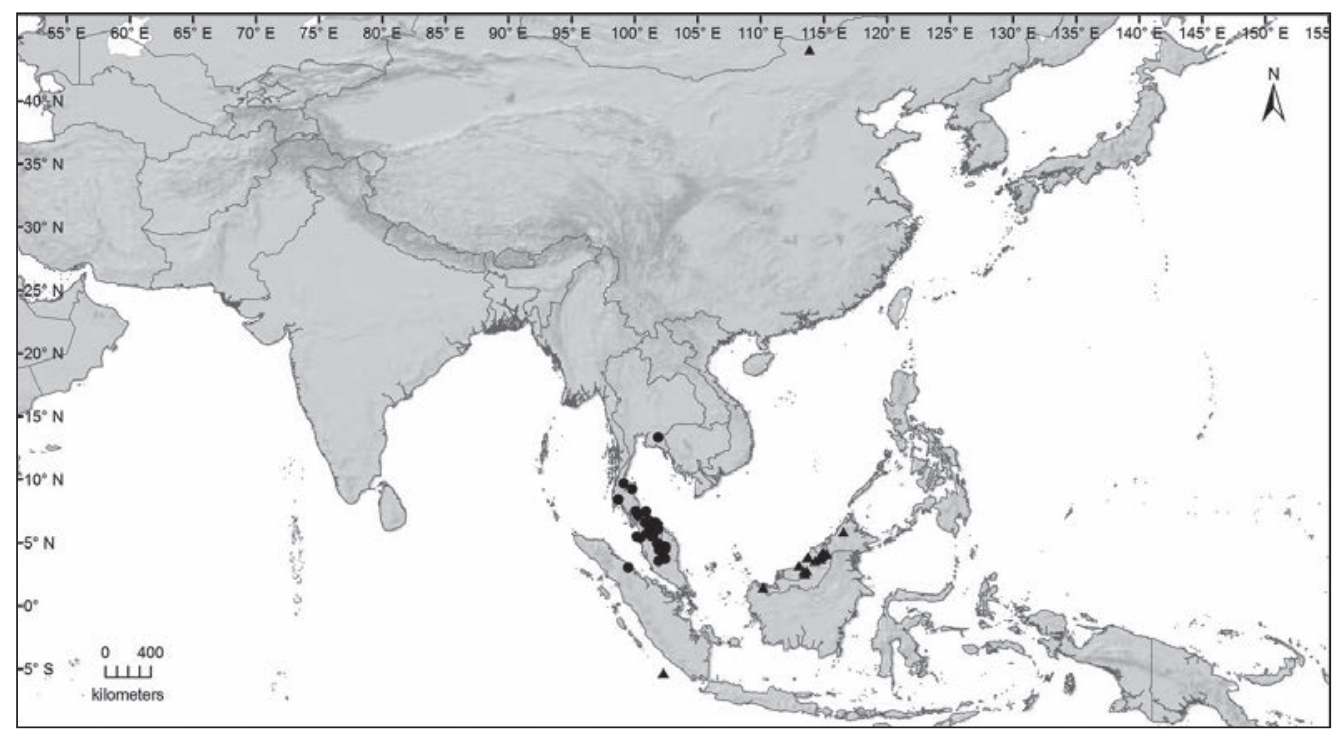

Fig. 9. Distribution of Epithema membranaceum (King) Kiew (•) and Epithema sarawakense Hilliard \& B.L.Burtt $(\boldsymbol{\Delta})$.

1.25-3.5 × 1.3-2.3 mm; lobes 0.6-3.1 × 0.75-2.5 mm; indumentum outside as on pedicel, sub-glabrous inside, a few hairs in lobes. Corolla colour variable, white, pale pink, pale violet, pale blue or purple or whitish-purple with purple markings, 3.5-7 mm long; tube 2.5-5 × $2 \mathrm{~mm}$, lobes 1-1.9 mm long; pubescent outside, hairs hooked and straight to $0.1 \mathrm{~mm}$ long, with a band of villous hair inside. Stamen filaments 0.75-0.8 mm long; anther characters unknown; staminodes $0.6 \mathrm{~mm}$ long. Nectary of one lobe almost or entirely encircling ovary, $0.75-1 \times 2.2 \mathrm{~mm}$. Ovary spherical or subspherical, $0.75-1 \times 0.6-1 \mathrm{~mm}$, pubescent, hairs medium to high density, fine, hooked, rarely with one or two straight hairs, hyaline, on upper portion of ovary; style 2-3.8 mm long, occasionally glabrous, pubescent on lower to mid-style, hairs hooked, to $0.13 \mathrm{~mm}$ long; stigma c. $0.4 \mathrm{~mm}$ wide. Fruit obovate to cylindrical, $2-3 \times 2-3 \mathrm{~mm}$; operculum $1 \mathrm{~mm}$ long, pubescence as for ovary. Seeds narrowly ovoid to ovoid, 0.3$0.5 \times 0.1-0.2 \mathrm{~mm}$, dark brown, pattern straight to spiralled, regular.

Distribution. Thailand, Peninsular Malaysia, Indonesia (Sumatra).

Habitat and ecology. Primarily on limestone, occasionally granite, in shaded areas of evergreen forests near waterfalls or streams. Found in lowland dipterocarp forests in Malaysia at 30-415 m altitude. Flowering and fruiting February, April-December.

Provisional IUCN conservation assessment. Least Concern (LC). This species is common and widespread although, as it mostly occurs on limestone and limestone habitats which are often threatened, its status should be monitored. 
Additional specimens examined. THAILAND: Chachoengsao: Khao Tak Groep, 6 Nov 1993, Larsen, K. et al. 44271 (AAU). Nakhon Si Thammarat: Kha Nom, Khuan Thong, Khao Krod Cave, 20 Dec 2006, Pooma, R. et al. 6486 (E); Nop Phitum, Khao Luang National Park, Khao Luang, 25 Oct 1991, Larsen, K. et al. 42584 (AAU); ibidem, 25 Oct 1991, Larsen, K. et al. 42579 (AAU). Narathiwat: 21 Dec 1968, Phusomsaeng, S. et al. 19 (BKF, E); Bacho National Park, 11 Oct 1991, Larsen, K. et al. 42312 (AAU, P); Sungai Padi, Chatvarin Falls, 18 Oct 1970-19 Oct 1970, Charoenphol, C. et al. 3976 (AAU, E, K, L); Pattani, Khao Chai Son, 23 Dec 2006, Pooma, R. et al. 6578 (E); Pattani, Sai Khao Waterfall, 9 Oct 1991, Larsen, K. et al. 42245 (AAU); ibidem, 20 Dec 1972, Santisuk, T. \& B.N. 451 (BKF). Phatthalung: Khao Chai Son, Road to Than Nam Yen, 23 Dec 2006, Pooma, R. et al. 6579 (BKF); Kao Ok Thalu, 21 Apr 1928, Kerr, A.F.G. 15775 (K). Songkhla: Khao Chang Lon, 24 Jul 1928, Kerr, A.F.G. 15893 (K); Saba Yoi, Tham Ru Nok Sak (Cave), 21 Oct 1991, Larsen, K. et al. 42471 (AAU, BKF); Tam ta Lord, 25 Nov 1990, Larsen, K. et al. 41713 (AAU). Surat Thani: Khan Thuli, 6 Sep 1931, Put 4118 (K). Yala: Bannang Sata, 26 Nov 1961, Suvanakoses, P. 1727 (BKF, K, L, P); Nam Tok Than To Falls, 10 Dec 1966, Sangkhachand, B. 1405 (BKF); Tahndo, Tahndo Falls Forest Park, 12 Nov 1986, Maxwell, J.F. 86-893 (L, PSU); Than To, Bang Lang National Park, 17 Jul 2004, Pooma, R. et al. 4298 (BKF).

MALAYSIA: Peninsular Malaysia: Kedah: Baling, 24 Apr 1987, Stone, B.C. et al. 8704245/1 (KLU); Kelantan: Gua Musang, 11 Aug 1971, Chin, S.C. 1404 (KLU); ibidem, 1 Nov 1967, Shimizu, T. \& Stone, B.C. 14427 (KYO); Kuala Betis, 15 Feb 2003, Kiew, R. RK5254 (E, SING). Pahang: Bentung, Bukit Chintamani, 3 Oct 1931, Henderson, M.R. 25010 (BO, SING); Gua Tipus, 10 Aug 1929, Henderson, M.R. 22580 (SING); ibidem, 15 Oct 1927, Henderson, M.R. 19406 (BO); Gunung Senyum, 30 Jul 1929, Henderson, M.R. 22382 (BO, SING); ibidem, 28 Nov 1984, Kiew, R. RK1590A (UPM); Kota Gelanggi, 28 Nov 1984, Kiew, R. RK1584 (UPM); Merapoh, Gua Layang, 13 Aug 1971, Chin, S.C. 1514 (E, K); Taman Negara, Batu Luas, 21 Oct 1982, Kiew, R. RK1231 (UPM); Taman Negara, Batu Luas, Gua Daun, 1 Oct 1984, Kiew, R. RK1347 (UPM); Taman Negara, Gua Luas, Wilkie, P. FRI52899 (E). Penang: 1822, Wallich, N. 9080 (K). Perak: Grik, Temenggor Dam, 9 Sep 1993, Turner, I.M. \& Yong 158 (SING); Grik, Temenggor Dam, Sungei Singor, 8 Nov 1993, Saw, L.G. FRI39945 (K).

INDONESIA: Sumatera Utara: Asahan, 12 May 1927-21 May 1927, Bartlett, H.H. 7740 (US).

Notes. This species is usually quite delicate and distinctive in its vegetative form. Usually the lower and middle petioles are $0.7-1.5$ times the length of the leaves which are all cordate-ovate with sub-cordate to sub-auriculate leaf bases. The petiole/ leaf length ratio decreases up the plant as in all petiolate species of Epithema, but even the uppermost petioles often appear long in comparison to other species. The curved asymmetric upper leaves of some plants are also seen in Epithema steensii and occasionally in E. tenerum. While peduncles usually originate in the axils of terminal leaves, a small number of specimens also have peduncles arising from the base of the petioles of the terminal leaves.

Epithema membranaceum can be similar to E. parvibracteatum and more rarely to E. saxatile. The similarity to Epithema parvibracteatum is discussed under that species. Label data suggests that Epithema membranaceum co-occurs with E. saxatile on Gunung Senyum and at Batu Luas, both in Pahang, Peninsular Malaysia. Epithema membranaceum can be separated from E. saxatile by the size of the bract, pubescence on the style and shorter hairs on the ovary/operculum. 
11. Epithema parvibracteatum Hilliard \& B.L.Burtt, Edinburgh J. Bot. 54: 112 (1997). - TYPE: Malaysia, Selangor, Batu Caves, 60 m, 3 November 1953, Sinclair, J. 7821 (holotype E). (Fig. 10)

Herb 6.5-19.6 cm high, caulescent, indumentum of strigose and setose to hispid hairs, to $0.8-1.1 \mathrm{~mm}$ long on stem, petioles, upper leaf surfaces and peduncles; stem 0.8$4.4 \mathrm{~mm}$ wide with two nodes, internodes $1.2-3.5 \mathrm{~cm}$ long. Leaves membranous or strongly membranous, petiolate; petiole of lowest leaf $0.8-5 \mathrm{~cm}$ long, upper petioles 0.4-2 cm long, densely hairy; blade of the lowest leaf 2.6-11.3 × 1.8-8 cm, upper leaf blades $2.3-8.5 \times 1.3-6 \mathrm{~cm}$, leaves frequently asymmetrical, one side up to 1.4 times wider than the other, apex acute to broadly acute, base shallowly auriculate to obtuse, inserted evenly on petiole, margin sub-entire to dentate, bidentate or serrate; upper surface often with two clearly different lengths of hair at medium to high density; lower surface drying white-green, longest hairs primarily on veins and at margins. Inflorescences $1-5$ per plant; peduncles (0.2-)0.5-10.8 cm long, terminal and in leaf axils, hispid; bracts small, rarely sub-cucullate or trullate, 1-7 $\times 2-7 \mathrm{~mm}$, margin often deeply lobed with 3 lobes, but also dentate; lower surface densely hairy with hairs to $0.6 \mathrm{~mm}$ long, upper surface with primarily straight hairs to $0.6 \mathrm{~mm}$ long, mostly denser and longer towards margins; pedicels 0.8-4.2 mm long, sparsely to densely hairy, hairs hooked and to $0.2 \mathrm{~mm}$ long, also with or without longer straight hairs to $0.6 \mathrm{~mm}$ long. Calyx 3.5-4.3 mm; tube 1.5-2.9 × 1.7-2.7 mm, lobes $0.8-2.9 \times 0.6-1.9 \mathrm{~mm}$; densely hispid throughout or mainly on lobes outside, hairs to $0.7 \mathrm{~mm}$ long; pubescent inside, hairs straight, on lobes. Corolla white, 4.2-5.4 mm long; tube cylindrical, 2.9-3.8 × 1-1.5 mm; lobes 0.8-1.3 mm long, margin entire; with few hooked hairs inside or with ring of villous hair. Stamens $1.1-1.6 \mathrm{~mm}$ long; filaments $0.6-1 \mathrm{~mm}$ long; anthers 0.5-0.6 mm long; staminodes $0.75-1 \mathrm{~mm}$ long. Nectary 1-3 discrete lobes, if three the third lobe is small, margin almost entire or undulate, $0.6-1.3 \times 0.3-1.9 \mathrm{~mm}$. Ovary spherical or sub-cylindrical, 1-1.3 $\times 0.9-1 \mathrm{~mm}$, sub-glabrous to densely pubescent or pilose, hairs straight and/or hooked, dense on upper ovary, to $0.2 \mathrm{~mm}$ long; style 1.9-3.2 mm long, glabrous or with few straight and/or hooked hairs; stigma c. $0.3 \mathrm{~mm}$ wide. Fruit obovate to cylindrical to sub-spherical, 1.5-2.8 $\times 1.9-2.5 \mathrm{~mm}$; operculum 0.4-0.9 mm long, indumentum as on ovary, hairs may be densest at the very top of the ovary. Seed narrowly to broadly ovoid, $0.3-0.5 \times 0.1-0.2 \mathrm{~mm}$, light brown to medium brown, pattern almost straight to spiralled, reasonably regular.

Distribution. Peninsular Malaysia.

Habitat and ecology. On limestone or other rocks, in shade, often in crevices of limestone, at 60-150 m altitude. Flowering and fruiting February, May, July-November.

Provisional IUCN conservation assessment. Least Concern (LC). This species is fairly widespread in Peninsular Malaysia although, as it mostly occurs on limestone and limestone habitats which are often threatened, its status should be monitored. 


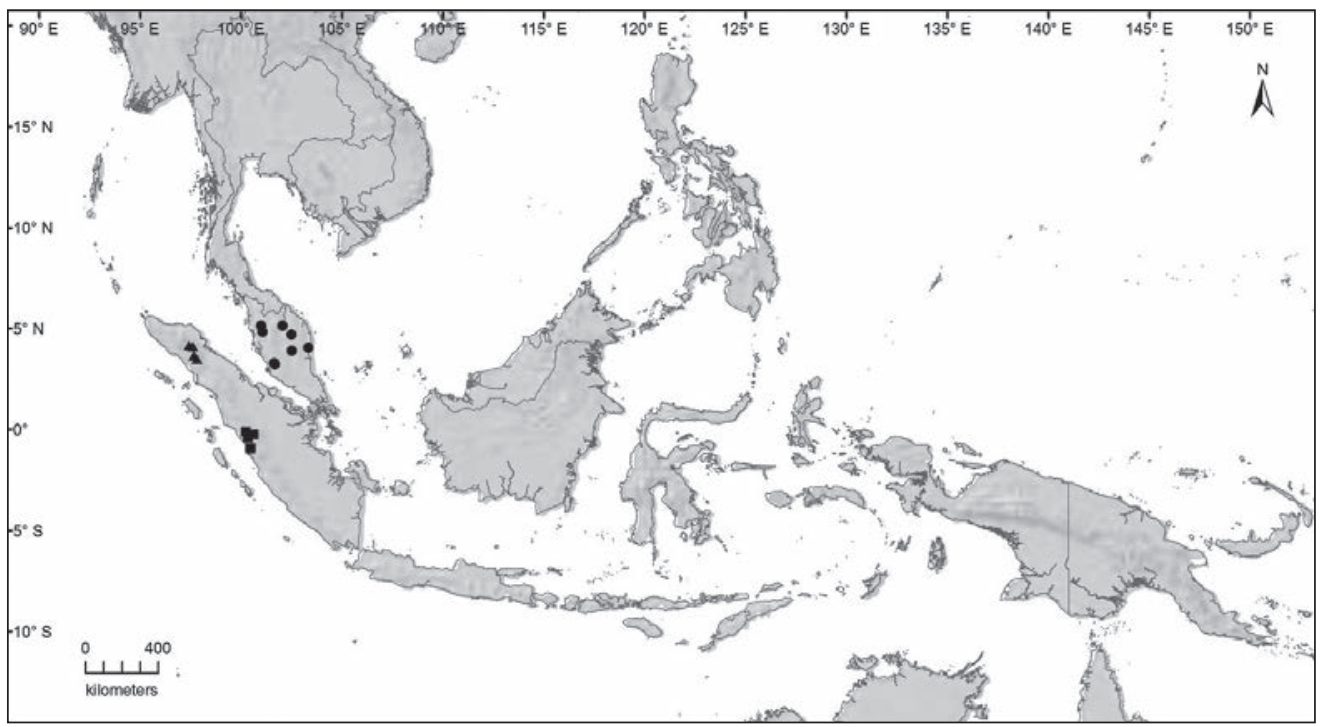

Fig. 10. Distribution of Epithema parvibracteatum Hilliard \& B.L.Burtt (•), Epithema steenisii Hilliard \& B.L.Burtt ( $\mathbf{\Delta})$ and Epithema strigosum (C.B.Clarke) Hilliard \& B.L.Burtt ( $\mathbf{\square})$.

Additional specimens examined. MALAYSIA: Peninsular Malaysia: Kelantan: Ulu Kelantan, Bertram, 30 Jul 1962, Unknown 147 (K, L). Pahang: Kota Gelanggi, Aug 1891, Ridley, H.N. s.n. (SING); Kuantan, Kuantan-Kemaman Road, 19 Jul 1979, Kochummen, K.M. FRI26221 (L); Taman Negara, Batu Subuh, 8 Oct 1984, Kiew, R. RK1498 (UPM). Perak: Hulu Perak, Lenggong, Kpg. Gua Badak, 28 Oct 2008, Imin, K. FRI63208 (SING); Kamuning, Feb 1904, Ridley, H.N. 11883 (K). Selangor: Kanching, Bukit Anak Takun, 4 Oct 1969, Stone, B.C. 8830 (KLU); ibidem, 29 May 1970, Stone, B.C. \& Mahmud 8400 (KLU). Selangor: Kanching, Bukit Takun, 27 Sep 1970, Chin, S.C. 392 (KLU); ibidem, 24 Oct 1967, Shimizu, T. \& Stone, B.C. 13739 (KYO).

Notes. The distinguishing characters for this species are a small bract and the dense and long hair covering, particularly on the peduncles, pedicels and calyx. In addition, the corolla is quite small, only $4.2-5.4 \mathrm{~mm}$ long. There is considerable variation in the ovary/operculum hair. This variation was observed both within a single inflorescence and between specimens. Variation in the type of hair found on the inside of the corolla is unusual and, apart from Epithema parvibracteum, is only seen in E. horsfieldii and E. rennellense.

The larger plants are rather similar to Epithema membranaceum and some also to E. saxatile. The specimens of Epithema parvibracteatum, however, have an extremely reduced bract and a hispid indumentum on the inflorescence.

12. Epithema philippinum (Hilliard \& B.L.Burtt) Bransgrove, stat. nov. - Epithema strigosum subsp. philippinum Hilliard \& B.L.Burtt, Edinburgh J. Bot. 54: 113 (1997). 
- TYPE: Philippines, Mindanao, Surigao, Mt Kabatuan, 83 m, 18 March 1949, Mendoza, D.R. \& Convocar, P.P. 297 [PNH10434] (holotype PNH; isotype A). (Fig. 6) Herb 6-13 cm high, usually acaulescent with leaves arising near ground level; stem 1-2.5 mm wide with 0-1 nodes, internode if present 1.4-2.3 cm long; stem densely pubescent, hairs to $0.5 \mathrm{~mm}$ long. Leaves strongly membranous, petiolate, with one or more inflorescences opposite each solitary leaf; petioles 2-20 cm long; blade 3-11 $\times 2.4-4.4 \mathrm{~cm}$, cordate, symmetrical, apex and tip acute, base cordate to truncate, inserted evenly on petiole, margin dentate and/or bidentate or crenate; upper surface weakly to strongly strigose, sometimes with setose hairs, hairs straight, hooked or a combination of both, to $0.7 \mathrm{~mm}$ long; lower surface pubescent, hairs to $0.4 \mathrm{~mm}$ long. Inflorescences 1-2 per plant; peduncles 1.6-10.5 cm long, originating opposite solitary leaves, strigose and setose, hairs dense, to $0.6 \mathrm{~mm}$ long; bracts cucullate but not enclosing entire inflorescence, 3-8 $\times 3-8 \mathrm{~mm}$, margin dentate; lower surface strigose, sometimes with two distinct lengths of hair or layers of hair visible, hairs to $0.4 \mathrm{~mm}$ long; upper surface strigose, on entire surface but denser towards margins, hairs to $0.3 \mathrm{~mm}$ long; pedicels $1.2-3.8 \mathrm{~mm}$ long, pubescent or hispid, hairs primarily straight, to $0.4 \mathrm{~mm}$ long. Calyx cylindrical, 3.5-5.5 mm long; tube $2.3-3 \times 1.5-3 \mathrm{~mm}$; lobes $1.2-2.5 \times 0.8-1.3 \mathrm{~mm}$; outside with a minute hooked pubescence and longer, primarily straight hairs covering the entire calyx or only on lobes, straight hairs $0.2-$ $0.4 \mathrm{~mm}$ long, hooked hairs $0.13-0.3 \mathrm{~mm}$ long; hairs on lobes inside. Corolla bluish, 5-6 mm long; tube cylindrical, 3.7-4.2 × 1.2-3 mm; lobes 1.5-2 mm long; band of villous hair inside in upper tube. Stamens $1-1.5 \mathrm{~mm}$ long; filaments c. $0.7-1 \mathrm{~mm}$ long; anthers c. $0.5 \mathrm{~mm}$ long; staminodes $0.7-1 \mathrm{~mm}$ long. Nectary of two discrete lobes nearly encircling ovary, 0.8-1.5 × 0.7-1.5 mm. Ovary sub-cylindrical or subspherical, $0.7-1.3 \times 0.7-1.2 \mathrm{~mm}$, upper part of ovary densely pubescent to pilose, hairs straight, to $0.1 \mathrm{~mm}$ long; style 3.7-4.6 mm long, glabrous or with few straight hairs at base; stigma c. $0.4 \mathrm{~mm}$ wide. Fruit sub-cylindrical or sub-spherical, 1.9-2.8 $\times 2-2.5$ $\mathrm{mm}$; operculum $0.6-0.9 \mathrm{~mm}$, indumentum as on ovary. Seed narrowly ovoid to ovoid, $0.4-0.5 \times 0.1-0.2 \mathrm{~mm}$, medium to dark brown, pattern straight to spiralled, regular, infrequently splitting and merging.

Distribution. Philippines (Mindanao).

Habitat and ecology. Lithophytic, probably on limestone in humid, shady areas. Recorded at $83 \mathrm{~m}$ altitude. Flowering and fruiting March.

Provisional IUCN conservation assessment. Data Deficient (DD). This species is only known from two quite old collections and the current status of the species is unknown. The area where these collections were made is still largely forested so the population may still be in good condition.

Additional specimen examined. PHILIPPINES: Mindanao: Agusan: Jabonga, Kitsarao, 14 Mar 1949, Mendoza, D.R. \& Convocar, P.P. 226 [PNH10394] (PNH). 
Notes. Epithema philippinum is most similar to E. strigosum. See discussion under Epithema strigosum regarding the differences between E. philippinum and E. strigosum.

Plants of Epithema philippinum that only have one leaf opposite one or more peduncles could also be confused with E. horsfieldii but is most easily distinguished by the straight hairs on the ovary and operculum in E. philippinum (hooked in E. horsfieldii).

13. Epithema pusillum (C.B.Clarke) Bransgrove, stat. nov. - Epithema carnosum var. pusillum C.B.Clarke, Monogr. Phan. 5(1): 178 (1883). - Epithema dentatum var. pusillum (C.B.Clarke) Hilliard \& B.L.Burtt, Edinburgh J. Bot. 54: 112 (1997). TYPE: India, Bombay, Stocks s.n. (holotype K). (Fig. 3).

Herb 4.5-12 cm high, caulescent, indumentum of straight and hooked hairs; stem $1 \mathrm{~mm}$ wide with two nodes $1.2-4 \mathrm{~cm}$ apart and $1-8 \mathrm{~cm}$ to the first node, stem sub-glabrous to sparsely pubescent, $0.2 \mathrm{~mm}$ long. Leaves thinly membranous, petiolate; petiole of lowest leaf $1-2 \mathrm{~cm}$ long, petioles of upper leaves $0.1-1.8 \mathrm{~cm}$ long, pubescent, hairs to $0.2 \mathrm{~mm}$ long; blade of the lowest leaf $4.3-6 \times 1.8-4.5 \mathrm{~cm}$, upper leaves $2-6.5 \times$ $1.2-4.8 \mathrm{~cm}$, lowest leaf broadly ovate, upper leaves ovate, all symmetrical, apex acute to rounded, base sub-cordate to truncate, inserted evenly on petiole, margin serrate; upper surface weakly strigose, hairs sparse, hyaline and/or white, $0.4 \mathrm{~mm}$ long; lower surface sub-glabrous to sparsely pubescent with primarily hooked hairs to $0.5 \mathrm{~mm}$ long. Inflorescences 1-3 per plant; peduncles 1-2.5 cm long, usually originating from axils of terminal leaves, indumentum sparse, strigose, straight hairs to $0.3 \mathrm{~mm}$ long, or with a sparse to dense minute hooked pubescence; bracts small and sub-cucullate to cucullate but not enclosing entire inflorescence, 4-8 $\times$ 4-9 mm, margin sub-entire with occasional dentation; lower surface glabrous or sub-glabrous; upper surface glabrous; pedicels 1.9-2.5 mm long, glabrous or with a minute, sparse pubescence. Calyx 2.1$4.6 \mathrm{~mm}$ long; tube $0.8-2.5 \times 1.7-2.5$; lobes $1-2.1 \times 0.7-2.1 \mathrm{~mm}$; usually glabrous or with a minute hooked pubescence, sometimes with additional straight and/or hooked hairs to $0.33 \mathrm{~mm}$ long outside; glabrous inside. Corolla white, c. $6 \mathrm{~mm}$ long; tube c. $4.6 \mathrm{~mm}$ long; lobes c. $1.4 \mathrm{~mm}$ long; glabrous outside, with villous band of hair inside in upper half. Stamen and staminode characters unknown. Nectary of one lobe, almost encircling the ovary, c. $0.8 \times 0.8-1.8 \mathrm{~mm}$, margin entire or sub-entire. Ovary subcylindrical to sub-spherical, $0.5-1 \times 0.5-1 \mathrm{~mm}$, glabrous; style c. $4 \mathrm{~mm}$ long; stigma c. $0.5 \mathrm{~mm}$ wide. Fruit sub-cylindrical, $1.5-2.3 \times 2.1-2.3 \mathrm{~mm}$; operculum c. $0.6 \mathrm{~mm}$, glabrous. Seed elliptic to fusiform, $0.4-0.5 \times 0.1-0.2 \mathrm{~mm}$, pattern partially spiralled to spiralled.

Distribution. India (Maharashtra).

Habitat and ecology. Lithophytic and epiphytic (on at least Dysoxylum sp.) in humid, probably shaded, places. Recorded from $739 \mathrm{~m}$ altitude. Flowering and fruiting September. 
Provisional IUCN conservation assessment. Data Deficient (DD). This species is only known from three collections, none of which were collected recently. The area where these collections were made has been heavily deforested and a survey of the status of this species is, therefore, vital.

Additional specimens examined. INDIA: Maharashtra: Ratnagiri, Amboli Ghat, Temple Point, 3 Sep 1968, Kulkarni, B.G. 108673 (E); Savantwadi, 30 Sep 1978, Almeida, S.M. s.n. (E).

Notes. Epithema pusillum is easily separated from the species under which it has been included as a variety, E. dentatum (now E. ceylanicum) and E. carnosum, by its glabrous ovary. Epithema pusillum is only known from central, western peninsular India. The specimen from Amboli Ghat is not as fine as the other two specimens seen here, but the ovary and the fruit, however, are consistently glabrous.

14. Epithema rennellense Hilliard \& B.L.Burtt, Edinburgh J. Bot. 54: 112 (1997). TYPE: Solomon Islands, Rennell Island, 18 August 1962, Dissing, H. 2738 (holotype $\mathrm{E}$; isotypes $\mathrm{E}, \mathrm{C})$.

Herb 12-27 cm high, often with an acaulescent appearance with first leaves arising near ground level, indumentum of short pubescent hairs and longer strigose or hispid hairs, the latter up to $0.8 \mathrm{~mm}$ long on petioles, upper leaf surfaces, peduncles, pedicels and outer calyces; stem 2-4.4 mm wide with one or two nodes, internodes 3.5-11.7 cm long. Leaves membranous to strongly membranous, petiolate, leaves solitary; petiole of lowest leaf 7-13 cm long, upper petioles $2.5-11.5 \mathrm{~cm}$ long; blade of lowest leaf 6-11.5 × 4.2-9.3 cm, upper leaf blades 4-14 × 3.4-10 cm, all blades cordate to oblong cordate or sub-orbicular, symmetrical or not, if asymmetrical, one side up to 1.4 times wider than the other, apex acute to rounded, base shallowly auriculate to rounded, inserted evenly on petiole or slightly offset, margin dentate, bidentate or serrate; lower surface light green or drying white-green, often finely pubescent or hispidulous. Inflorescences 1-2 per plant; peduncles 2-21 cm long, originating opposite single leaves or occasionally in leaf axil; bracts sub-cucullate to cucullate, occasionally small, enclosing the entire inflorescence or portion thereof, 3-13 $\times 4-10$ $\mathrm{mm}$, margin entire with occasional crenate or dentate lobes to dentate; upper surface sub-glabrous to sparsely pubescent, hairs to $0.2 \mathrm{~mm}$ long, usually on upper portion of bract; pedicels 1.3-5 mm long. Calyx light green, 4.2-7.5 mm long, tube (1.7-)3-4.2 × (1.5-)2-3 mm, lobes 1.5-5 × 1-1.5 mm; glabrous to sparsely strigose on lobes inside, hairs straight or straight and hooked, to $0.13 \mathrm{~mm}$ long. Corolla white, $10.4-15 \mathrm{~mm}$ long; tube 6-11 × 2-3.3 mm; lobes 3-4.6 mm long, margin uneven/undulate; glabrous or sub-glabrous or with a band of villous hair inside. Stamens 1.2-3.1 mm long; filaments $0.7-2.1 \mathrm{~mm}$ long; anthers $0.5-1 \mathrm{~mm}$ long; staminodes $0.8-1.5 \mathrm{~mm}$ long. Nectary variable, one lobe (not entire) encircling ovary or with two discrete lobes not encircling ovary, 0.6-1.6 × 0.7-3.5 mm, margin entire to undulate. Ovary 1-1.6 × 0.8-1.6 mm, sub-glabrous or pubescent at sparse to medium density on upper portion 
of ovary, hairs hooked or both straight and hooked, $0.04-0.3 \mathrm{~mm}$ long; style to 8.8 $\mathrm{mm}$ long, glabrous or with few hairs at base, hairs straight and hooked; stigma c. 0.5 mm wide. Fruit obovate, cylindrical or sub-cylindrical, 2.5-4 × 1-3.3 mm; operculum 0.7-1.3 mm, indumentum as ovary, but often on top of operculum or densest on top of operculum. Seed narrowly ovoid to ovoid, $0.4-0.8 \times 0.1-0.2 \mathrm{~mm}$, light to dark brown, pattern straight to spiralled, reasonably regular, walls somewhat thickened and rigid.

Distribution. Solomon Islands (Rennell Island).

Habitat and ecology. Growing on well-drained hillsides over limestone (Rennell Island is a limestone, coral atoll) at 20-90 m altitude.

Provisional IUCN conservation assessment. Least Concern (LC). Although this species has not often been collected, and it is endemic to an island that is only $660 \mathrm{~km} 2$, the island is still largely forested and there is little evidence that the the population is under serious threat.

Additional specimens examined. SOLOMON ISLANDS: s.l. 1894, Officers of H.M.S. “Penguin” (K). Rennell Island: 21 Aug 1962, Dissing, H. 2798 (C); 6 May 1968, Sirute'e, B. et al. BSIP9621 (K, L, SING); Hutuna, 25 Mar 1965, Wolff, T. 3004 (C); Matangi Area, 17 May 1969, Gafui, I.H. \& collectors BSIP14759 (K, L, SING); Nuipani, 14 Mar 1965, Wolff, T. 3025 (C); Tuhungganggo, 24 May 1969, Gafui, I.H. \& collectors BSIP14763 (K, L, SING).

Notes. This species is similar to Epithema longipetiolatum and E. strigosum in the leaves arising at ground level. Epithema rennellense is also somewhat similar to $E$. dolichopodum but differs in peduncle length/plant height ratio, leaf shape and leaf margin, the length of the calyx and the corolla, the ovary/operculum hair and the size of the seed. The variation in the shape of the hair on both the ovary and the operculum, between hooked and both straight and hooked, can be seen on different fruit from the one plant. The variation in corolla hair is unusual and is seen in few other species, none of which are acaulescent species. Epithema rennellense has the largest seed of all species of Epithema and, along with E. longitubum, the largest flowers.

15. Epithema sarawakense Hilliard \& B.L.Burtt, Edinburgh J. Bot. 54: 113 (1997). TYPE: Malaysia, Sarawak, Fifth Division, Sungai Medalam, Gunung Buda, 20 June 1975, Burtt, B.L. 8336 (holotype E; isotype SAR). (Fig. 9)

Herb 25-40 cm high, sprawling or caulescent, indumentum of pubescent or strigose and setose hairs, to $0.7 \mathrm{~mm}$ long on the lower leaf surface and calyx, to $1 \mathrm{~mm}$ long on the upper leaf surface; stem 1.2-5 mm wide with 2 to 4 nodes, internodes $1-8 \mathrm{~cm}$ apart long. Leaves membranous to strongly membranous, petiolate, primarily solitary, rarely with one or two sets of opposite leaves per plant; petioles 1-7 cm long; blade 2.2-17 × 1.4-13.2 cm, often cordate, but can be sub-orbicular, elliptic or sub-oblong, 
symmetrical or not, if asymmetrical, one side up to 1.5 times wider than the other, apex acute to rounded, occasionally almost truncate, base usually (sub-)cordate but also to truncate, inserted evenly on petiole or not, margin sub-entire to dentate or bidentate; upper surface sparsely to densely strigose or villous, occasionally sub-glabrous; lower surface may dry grey-green, glabrous to villous. Inflorescences 3-11 per plant; peduncles $0.5-16 \mathrm{~cm}$ long, 1-5 arising in sequence opposite solitary leaves or rarely in the axils of upper opposite leaves; bracts cucullate, usually completely enclosing inflorescence, $10-32 \times 6-12 \mathrm{~mm}$, margin dentate or irregularly dentate; lower surface with hairs to $0.5 \mathrm{~mm}$ long; upper surface often glabrous; pedicels $1.3-4.6 \mathrm{~mm}$ long, often glabrous. Calyx 3.3-6.6 mm long; tube 1.5-3.5 × 1-3.1 mm, lobes 1.5-3.8 × 0.6-2.3 mm; frequently sub-glabrous to finely pubescent outside (short, hooked hairs), occasionally with sparse strigose hairs; inside glabrous or with few straight hairs, 0.1 mm long, in tips of lobes. Corolla white and pale blue to purple, pink, or mauve, may have darker markings on the lobes, 8.7-11 mm long; tube 6-7 × 1.5-2.5 mm, lobes $2.7-4.2 \times 1.5-2.1 \mathrm{~mm}$, margin slightly fimbriate. Stamens $3 \mathrm{~mm}$ long; filaments 1.5 mm long; anthers $1.5 \mathrm{~mm}$ long; staminodes 1.5-1.8 mm long. Nectary a single lobe, partially to completely encircling ovary, $1-1.3 \times 1.2-2.5 \mathrm{~mm}$, margin almost entire to undulate, occasionally almost dividing into smaller lobes. Ovary sub-cylindrical, 0.8$1 \times 0.7-0.9 \mathrm{~mm}$, usually glabrous, occasionally ovary pubescent with hooked hairs on upper ovary, hairs to $0.1 \mathrm{~mm}$ long; style c. $6 \mathrm{~mm}$ long, slightly wider at base, glabrous; stigma c. $0.4 \mathrm{~mm}$ wide. Fruit obovate-cylindrical, 2-3 $\times 1 \mathrm{~mm}$; operculum glabrous, occasionally sub-glabrous, indumentum as on ovary. Seed narrowly ovoid to ovoid or somewhat sigmoid, $0.4-0.5 \times 0.1-0.2 \mathrm{~mm}$, light brown to dark brown, pattern straight or almost so, occasionally partially spiralled to spiralled.

Distribution. Malaysia (Sabah, Sarawak), Indonesia (Kalimantan, Sumatra: Pulau Enggano).

Habitat and ecology. Lithophytic on limestone (but substrate not always reported). Humid, shady areas, beside streams, limestone outcrops, cave entrances. Occasionally found on wood or on soil over rock. At 30-1250 m altitude. Flowering and fruiting year round.

Provisional IUCN conservation assessment. Least Concern (LC). This species is widespread with many of the collections having been made in the protected areas of Gunung Mulu National Park and Niah National Park.

Additional specimens examined. MALAYSIA: Borneo: Sabah: Tambunan, Crocker Range, Tambunan/Ranau Road, 5 Mar 1995, Sugau, J. JBS96 (E). Sarawak: 1st Division, Jambusan, Gunung Batu , 6 Oct 1977, Martin, P.J. S.39273 (E, K, L); Baram District, Jun 1894, Haviland, G.D. \& Hose, C. 3525 R (K); Baram District, Batu Gading, 5 Jan 1965, van Niel, J.P. 3554 (L); Baram District, Gunung Mulu National Park, Bukit Binarat, 28 Apr 1985, Moktar, A. et al. S.49467 (E, K); Baram District, Gunung Mulu National Park, Deer Cave, 16 i 1978, Hansen, C. 17 (C, E, SAR); ibidem, 16 Jan 1978, Hansen, C. 17 (C, E); ibidem, 2 Oct 2007, Julia, S. et 
al. S.99303 (E); ibidem, 5 May 1978, Kiew, R. RK525 (E, UPM); ibidem, 16 Jan 1978, Nielsen, I. 17 (E); ibidem, 23 Jul 1987, Primack, R.B. S.42402 (K, L); Baram District, Gunung Mulu National Park, Gua Rusa, 30 Oct 1977, Argent, G.C.G. \& Kerby, R. 621 (E); Baram District, Gunung Mulu National Park, 22 Mar 1964, Hotta, M. 15281 (E, KYO, L); ibidem, 14 Mar 1964, Hotta, M. 14402 (E, KYO); ibidem, 19 Mar 1990, Yii, P.C. \& Abu Talib S.58618 (SAR); Miri, Mulu National Park, en route from HQ to Deer Cave, 20 Dec 1999, Imaichi, R. et al. 30 (SAR); Miri, Mulu National Park, Royal Mulu Resort, 6 Oct 2001, Julaihi S.86868 (SAR); Miri, Mulu National Park, Simons Cave, 27 Apr 1997, Haegens, R.M.A.P. \& Klazenga, N. 515 (SAR); Baram District, Gunung Mulu National Park, Melinau Gorge, 23 Jun 1962, Burtt, B.L. \& Woods, P.J.B. B. 2230 (E); ibidem, 20 Feb 1978, Nielsen, I. 416 (E); Baram District, Gunung Mulu National Park, Pala River, 14 Nov 1977, Argent, G.C.G. \& Collins, M. 733 (E); Baram District, Ulu Melinau, Aug 1958, Ashton, P.S. A. 339 (K); ibidem, Aug 1958, Ashton, P.S. A. 340 (K); Baram District, Ulu Sg. Tutoh, Batu Kalulong, 13 Apr 1997, Julaihi et al. S.76938 (SAR); Batu Niah, Nov 1932, Synge, P.M. S.565 (K); ibidem, Nov 1932, Synge, P.M. S.634 (K); Batu Niah, Gunung Subis, 28 Nov 1966, Anderson, J.A.R. et al. S.26090 (A, E, K, L, SING); ibidem, Jan 1961, Mohidin S.21608 (K); ibidem, 19 Aug 2002, Sabli, J. et al. S.89048 (SAR); Batu Niah, Niah Cave Park, 4 Jun 1962, Burtt, B.L. \& Woods, P.J.B. B.2003 (E); ibidem, 3 Jun 1962, Chew, W.-L. CWL.293 (K, L, SAR, SING); ibidem, 23 Dec 1999, Imaichi, R. et al. 38 (SAR); ibidem, 20 Aug 2006, Prieditis, N. s.n. (E); ibidem, 20 Aug 2006, Prieditis, N. s.n. (E); ibidem, 18 Apr 1978, Stone, B.C. 13730 (K, KLU, L); Belaga District, Bukit Merirai, Gua Pak Danum, 12 Jul 2005, Leong, P. et al. PL320 (SING); Belaga District, Ulu Merirai, 9 Jul 2005, Leong, P. et al. PL215 (SING); Belaga District, Ulu Merirai, Gua Tiang, 6 Jul 2005, Julia, S. et al. S.93376 (SING); Bintulu, Ulu Sg. Kakus, Bukit Sarang, 14 Mar 1965, Anderson, J.A.R. S.20957 (A, K); Bintulu, Ulu Sg. Kakus, Bukit Sarang, Batu Anyi, 5 Oct 2004, Julia, S. et al. S.94757 (SAR, SING); Buseau, 1890, Haviland, G.D. s.n. (K); Kapit, Melinau, 16 Jul 1961, Anderson, J.A.R. \& Keng, H. K100 (SAR); Kapit, Ulu Melinau, Gunung Api, 1971, Anderson, J.A.R. S.31770 (E, K, L, SING); ibidem, 10 Sep 1970, Chai, P. S.30372 (A, BO, K, L, SING); ibidem, 1970, Lehmann, P.F. PFL. 596/S. 30372 (E); ibidem, 7 Sep 1970, Lehmann, P.F. S.30091 (E, K, SAR); ibidem, 29 Mar 1990, Yii, P.C. \& Abu Talib S.58841 (SAR); ibidem, 29 Mar 1990, Yii, P.C. \& Abu Talib S.58841 (SAR); Lobang Rusa, 9 Jun 1975, Burtt, B.L. B. 8227 (E, SAR); Lobang Rusa, Sungei Melinau Paku, 9 Feb 1966, Chew, W.-L. CWL1017 (A, K, L, SING); Marudi District, The Pinnacles, Gunung Api, 17 Jun 1995, Beaman, J.H. 11727 (K); Miri, Gunong Mulu National park, Hidden Valley, 5 Apr 1978, Argent, G.C.G. et al. $905 b$ (E); Niah, 7 Oct 1954, Ahmad 1 (SING); Niah National Park, 12 Dec 1981, Rogstad, S.H. 728 (A). INDONESIA: Sumatra: Bengkulu: Pulau Enggano, Boea-boea, 11 Jun 1936, Lütjeharms, W.J. 4598 (L); Pulau Enggano, Malakoni, 26 Jun 1936, Lütjeharms, W.J. 5112 (BO, GH, K, L, P). Kalimantan: Peningin, Jaheri 1522 (BO).

Notes. Epithema sarawakense is usually quite distinctive with its near complete lack of opposite leaves and one to five infloresences arising from the base of the solitary petiole. Epithema sarawakense is often densely hairy, giving the dried leaves a greygreen colour. There are two forms, however, one of which is glabrous or sub-glabrous. The glabrous form is found almost exclusively in Gunung Mulu National Park in the environs of Deer Cave and the base of Gunung Mulu. While there are a small number of collections of the pubescent form from near Deer Cave, it has primarily been collected from Gunung Api, Gunung Buda, Gunung Benarat, along Sungai Melinau and in the Melinau Gorge. 
There are a small number of specimens that show some similarity to Epithema saxatile. These specimens have some opposite leaves but, in most cases, there are always solitary leaves with peduncles arising opposite the solitary leaf on the plant. These opposite leaves are unequal in size while Epithema saxatile has even-sized opposite leaves. In addition, the range of the corolla length of Epithema sarawakense is $8-11 \mathrm{~mm}$ while that of $E$. saxatile is $6.5-9.5 \mathrm{~mm}$ and the upper ovary/operculum of $E$. sarawakense is glabrous or sub-glabrous (hairs hooked) while the operculum of E. saxatile is always at least sparsely pubescent (hairs hooked). The specimens of Epithema sarawakense that are similar to E. saxatile are not segregated geographically from more typical E. sarawakense.

A small number of specimens from Pulau Enggano in Sumatra are included in Epithema sarawakense. While the distribution is difficult to explain, there are currently no characters to separate the specimens from the remainder of the species as found in Sarawak. They have shorter stems than is usual for Epithema sarawakense, thereby resembling E. strigosum, but they differ from E. strigosum in that the operculum is glabrous to sparsely pubescent with hooked hairs rather than dense and with straight hairs as in E. strigosum.

16. Epithema saxatile Blume, Bijdr. 738 (1826); Ridley, Fl. Malay Penins. 2: 539 (1923); B.L.Burtt, Thai For. Bull. (Bot.) 29: 94 (2001). - TYPE: Indonesia, Java, Blume, C.L. 1029 (lectotype L [L0003232], designated here; probable isolectotype U [without number]). (Fig. 11, 12)

Epithema carnosum auct. non Benth.: Barnett, Fl. Siam. 3(3): 205 (1962), p.p.

Herb 6-40 cm high, caulescent; stem 2-5 mm wide with 2-5 nodes, internodes $1.2-10.5 \mathrm{~cm}$ long, usually glabrous or sub-glabrous. Leaves membranous to strongly membranous, petiolate or with sessile upper leaves, rarely with one or two solitary upper leaves; petiole of lowest leaf 2.5-12 cm long, upper petioles 0-2.5 cm long; blade of lowest leaf 7-20 × 6.5-12.5 cm, upper leaves 4.5-13.5 × 3-8.5 cm, lowest leaf sub-spherical to broadly ovate, upper leaves variable, often narrowly ovate to almost oblong, but also cordate, broadly ovate or elliptic, symmetrical or not, if asymmetrical, one side up to 1.4 times wider than the other, apex acute to broadly acute, rarely rounded, base cordate to truncate, inserted evenly on petiole to distinctly offset (up to $2 \mathrm{~cm}$ ), margin sub-entire to dentate, bidentate or serrate (often all or degrees of these on one plant); upper surface green or dark-green, indumentum occasionally to $1.1 \mathrm{~mm}$ long; lower surface light green, whitish or occasionally purplish. Inflorescences 1-10 per plant; peduncles 2-17 cm long, indumentum occasionally hispid, to $0.7 \mathrm{~mm}$ long; bracts cucullate, usually enclosing the entire inflorescence, 13-35 × 8-12 mm, margin usually dentate, occasionally sub-entire with one or two teeth or lobes; lower surface usually sparsely to densely strigose, hairs straight or primarily straight, $0.3-0.5 \mathrm{~mm}$ long; upper surface glabrous or sub-glabrous, hairs towards margins of bract; pedicels 1.1-4.2 mm long. Calyx 3.3-5 mm long; tube 1.6-3.3 × 1.5-2.1 mm; lobes 1.5-2.7 × 


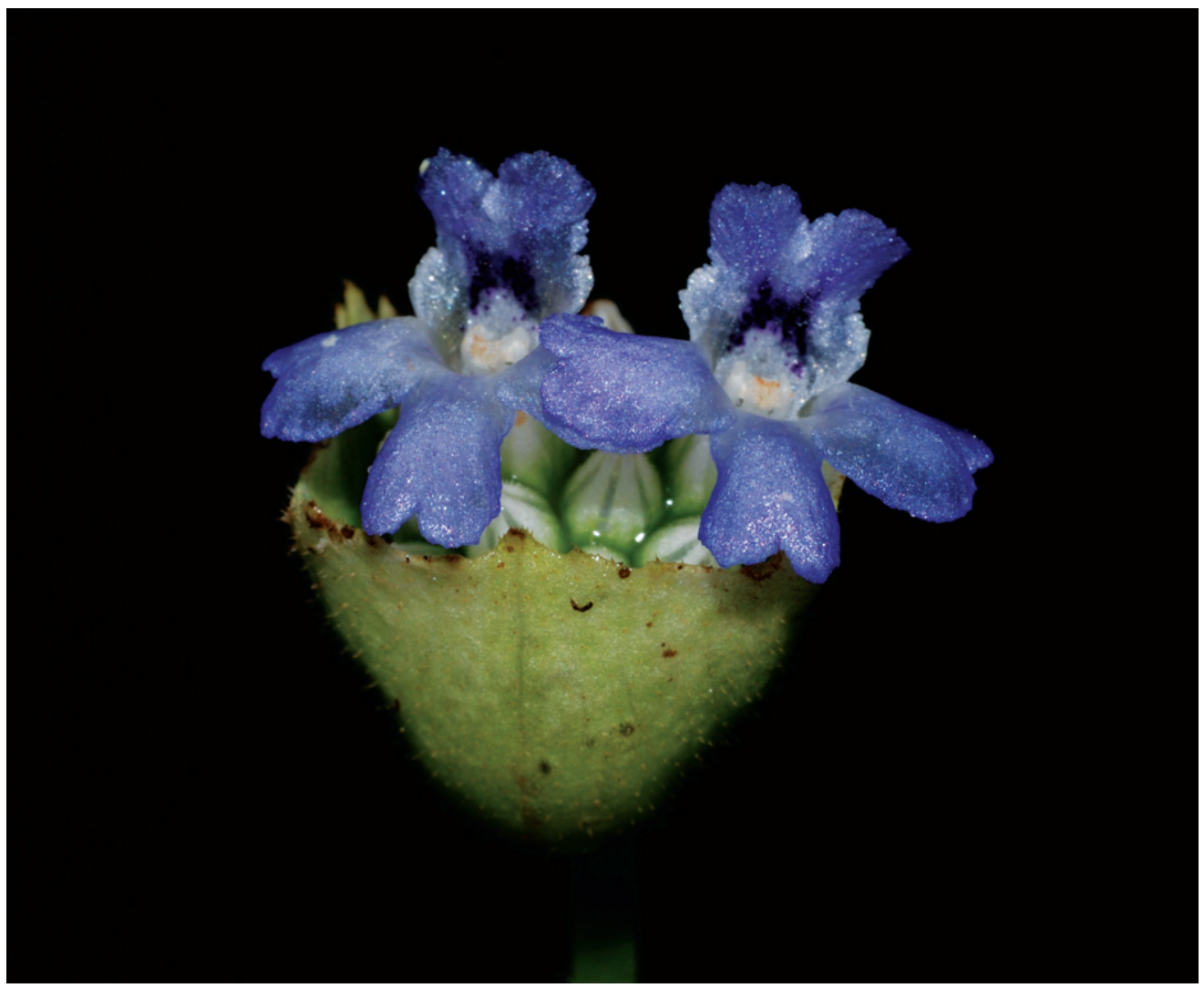

Fig. 11. Epithema saxatile Blume. Flowers and cucullate bract. (Photo: David Middleton)

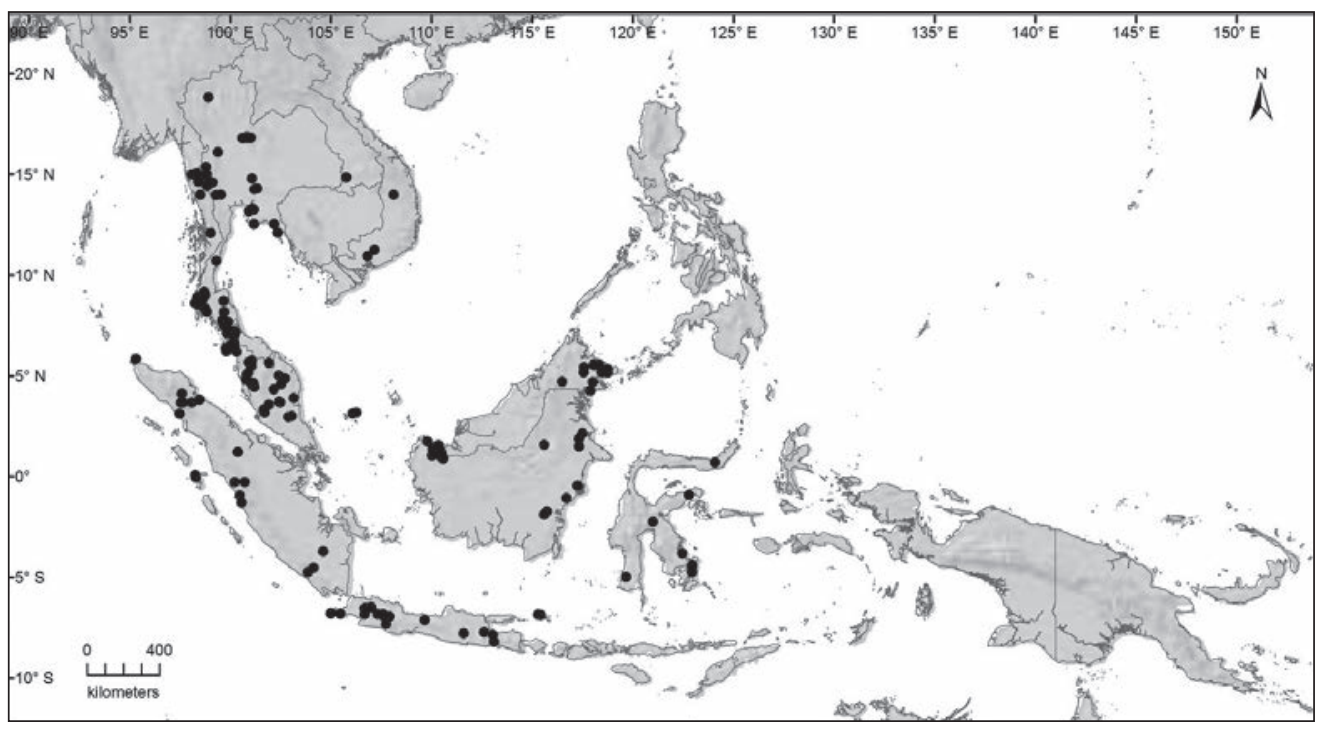

Fig. 12. Distribution of Epithema saxatile Blume (•). 
0.6-0.9 mm, often glabrous at maturity, hairs to $0.5 \mathrm{~mm}$ long, glabrous inside. Corolla white, pink, blue, purple, or purple and white, frequently with highly variable purple markings on the upper lip, 6.5-9.5 mm long; tube 5-7 × 1.5-2.5 mm; lobes 1-3 $\times$ 1.7-2.3 mm; band of villous hair up to $1 \mathrm{~mm}$ wide inside. Stamen filaments 1.3-2.1 mm long; anthers c. $0.6 \mathrm{~mm}$ long; staminodes 1.3-2.1 mm long. Nectary apparently absent or with one or two lobes partially to entirely encircling ovary, margin undulate, 0.7-1.3 × 0.6-2 mm. Ovary sub-cylindrical or sub-obovate, 0.6-1.5 × 0.5-1 mm, very sparsely to densely pubescent, hairs hooked, on the upper or very upper portion of ovary, hairs $0.04-0.63 \mathrm{~mm}$ long; style $2.9-5 \mathrm{~mm}$, glabrous or sparsely pubescent on the lower style; stigma $0.3 \mathrm{~mm}$ wide. Fruit cylindrical or obovate, 1.9-2.6 × 1.7-2.1 $\mathrm{mm}$; operculum $0.4-0.7 \mathrm{~mm}$ long, pubescence as ovary. Seed narrowly ovoid to ovoid, $0.3-0.5 \times 0.1-0.2 \mathrm{~mm}$, pattern stright to spiralled, reasonably regular.

Distribution. Myanmar, Thailand, Malaysia (Peninsular Malaysia, Sarawak, Sabah), Indonesia (Sumatra, Java, Kalimantan, Sulawesi).

Habitat and ecology. Shaded, humid areas, usually on or amongst limestone rocks or in limestone soil, near, by or in streams or rivers. Also found near or on the walls of cave mouth entrances. At 0-910 m altitude. Flowering and fruiting hugely variable.

Provisional IUCN conservation assessment. Least Concern (LC). This species is common and widespread.

Additional specimens examined. MYANMAR: Tenasserim, 1877, Gallatly, G. 1035 (SING); Tenasserim, Tavoy, Paungdaw, Aug 1961, Keenan, J. et al. 798 (E).

THAILAND: Chanthaburi: Nam Tok Phliu National Park, Nam Tok Phliu Waterfall, 30 Aug 1969, Maxwell, J.F. s.n. (AAU); Priu Waterfall, 12 Oct 1965, Chermsirivathana, C. 413 (BKF). Chiang Mai: Doi Sutep, 4 Aug 1912, Kerr, A.F.G. 2655 (K); Me Ta Chang, 17 Oct 1922, Kerr, A.F.G. 6381 (ABD, E, K). Chon Buri: Bahn Beung District, Ang Chang Nam, 1 Sep 1975, Maxwell, J.F. 75-958 (AAU, BKF, L); Pong NamRawn, 3 Sep 1956, Smitinand, T. 3489 (BKF, E); Sriricha, Chundaten Falls, 17 Aug 1974, Maxwell, J.F. 74-814 (AAU, BK, L). Chumphon: Bang Son, 11 Sep 1927, Put 1051 (ABD, K). Kanchanaburi: Between Huay Ban Kao and Kritee, 4 Jul 1973, Geesink, R. \& Phengklai, C. 6082 (BKF, E, L); ibidem, 12 Jul 1973, Geesink, R. \& Phengklai, C. 6231 (AAU, BKF, C, E, K, L, P). Kanchanaburi: Hindato, 24 Jul 1946, Kostermans, A.J.G.H. 1346 (BO, A, L, P); Dongyai, 14 Aug 1971, Phengklai, C. 2933 (BKF); Khaibuing, CP, BS \& BN 2997 (BKF, E, L); Kin Sayok, 13 Jul 1946, Kostermans, A.J.G.H. 1103 (A, BO, L, P, SING, US); Lai Wo, Toong Yai Naresuan Wildlife Reserve, Ban Saneh Pawng, 12 Aug 1993, Maxwell, J.F. 93-886 (CMU); Rintin, 31 Jul 1946, Kostermans, A.J.G.H. 1401 (A, BO, L, P, SING, US); Si Sawat, Hua Lum Kao Ngoo, 5 Jul 1973, Sutheeson, S. 2489 (BKF); Tawng Pa Poom, Huay Ban Khao, 12 Jul 1973, Maxwell, J.F. 73-285 (AAU). Khamphaeng Phet: Khlong Lan National Park, 21 Aug 1995, Parnell, J. et al. 95-335 (K). Krabi: 29 Mar 1930, Kerr, A.F.G. 19385 (K); Ao Luk, 21 Jun 2006, Williams, K. et al. 2033 (A); Nai Chong, 21 Oct 1979, Sutheeson, S. 5004 (BKF). Mae Hong Son: Muang, Nahng Rawng Falls, 16 Sep 1972, Maxwell, J.F. $72-362$ (L). Nakhon Nayok: Nang Rong Falls, 13 Aug 1968, Larsen, K. et al. 3363 (AAU, BKF, C, E, K, L, P, SING). Nakhon Si Thammarat: Thung Song District, Khao Tham Long, 31 Aug 1982, Shimizu, T. et al. T.28985 (BKF); Nop 
Phitum, Khao Luang National Park, Krung Ching Falls, 24 Sep 2010, Middleton, D.J. et al. 5524 (E); ibidem, 12 Feb 2005, Williams, K. et al. 1400 (E). Phangnga: Khao Lak, 21 Sep 1963, Smitinand, T. \& Sleumer, H.O. 1207 (BKF, L). Phangnga: Muang, Suan Somdet, 8 Dec 1999, Wongprasert, T. 9912-30 (BKF); Muang, Tham Pha Phueng, 15 Sep 2010, Middleton, D.J. et al. 5425 (E); Panga, Sep 1894, Curtis, C. s.n. (SING); ibidem, Aug 1893, Curtis, C. s.n. (SING); Pulau Tebun, 29 Dec 1918, Nur, M. 3585 (K, SING); Takuapah, 14 Jul 1972, Larsen, K. et al. 30967 (AAU, E); Tham Tong Lang, Shimizu, T. et al. T.29151 (BKF). Phatthalung: Khao Pu-Khao Ya National Park , 24 Sep 1986, Maxwell, J.F. 86-723 (A, BKF, L, PSU). Phitsanulok: N. Phitsanulok, Thung Salaeng Luang National Park, 22 Jul 1966, Larsen, K. et al. 722 (AAU, BKF, L); ibidem, 25 Jul 1973, Murata, G. et al. T.17092 (AAU, BKF, KYO, L); ibidem, Phusomsaeng, S. et al. 83 (BKF); ibidem, 30 Sep 1967, Tagawa, M. et al. T.11259 (KYO); Thung Salaeng Luang National Park, Kaeng Sopa Waterfall, 22 Oct 1984, Murata, G. et al. 38544 (BKF); ibidem, 22 Oct 1984, Murata, G. et al. T.38467 (BKF); Thung Salaeng Luang National Park, Kang So Pa Waterfall, 17 Sep 1990, Chantharanothai, P. et al. 90/303 (K); Thung Salaeng Luang National Park, Poi Waterfall, 22 Oct 1984, Murata, G. et al. T.38587 (BKF). Saraburi: Khao Muak Lek, 2 Oct 1963, Bin Rajab, M.K. 714 (KLU). Songkhla: Boripath Waterfall, 1990, Larsen, K. et al. 41243 (AAU); ibidem, 16 Aug 1984, Maxwell, J.F. 84-55 (A, PSU); Boriphat Falls National Park, 9 Nov 1990, Larsen, K. et al. 41243 (P). Surat Thani: Ban Kawp Kiep, 5 Aug 1927, Kerr, A.F.G. 13183 (ABD, E, K). Surat Thani: Khlong Phanom National Park, Middleton, D.J. et al. 4345 (BKF, E); Phanom, Khao Sok National Park, 6 Sep 2008, Middleton, D.J. et al. 4330 (E); Phanom, Khao Sok National Park, Chong Lom, 12 Dec 1979, Shimizu, T. et al. T.27103 (KYO); ibidem, 12 Dec 1979, Shimizu, T. et al. T.27111 (BKF, KYO); ibidem, 12 Dec 1979, Shimizu, T. et al. T.27100 (KYO); Phanom, Khao Sok National Park, Sii Ru Cave, 27 Feb 2006, Middleton, D.J. et al. 4055 (BKF, E); Sawng Pi Nawng, 20 Mar 1927, Kerr, A.F.G. 12385 (K). Satun: Khuan Don, Thale Ban National Park, Along cliff face from Ton Din Cave, 9 Sep 2010, Middleton, D.J. et al. 5350 (E); Thale Ban National Park, 27 Aug 1995, Larsen, K. et al. 46055 (AAU); Thung Wa, Than Plew Waterfall, 10 Sep 2010, Middleton, D.J. et al. 5368 (E). Trang: Ampoe Kao Kao, Kao Chom Lem, 2 Aug 1929, Rabil 318 (K). Trang: Ban Nam Phrai, 17 Dec 1979, Shimizu, T. et al. T.27551 (BKF, KYO, L); ibidem, 17 Dec 1979, Shimizu, T. et al. T.27571 (KYO); Chawng, 15 Mar 1959, Smitinand, T. \& Abbe, E.C. 6149 (BKF, K); Huay Yot, Wat Tham Iso, Middleton, D.J. et al. 4428 (E, SING); Lamphura, 15 Nov 1990, Larsen, K. et al. 41413 (AAU, BKF); ibidem, 16 Nov 1990, Larsen, K. et al. 41450 (AAU); Trang, Nam Tai, 11 Oct 1970, Charoenphol, C. et al. 3662 (AAU, E); ibidem, 11 Oct 1970, Charoenphol, C. et al. 3661 (AAU, BKF); Trat, Koh Chang, 7 Sep 1992, Niyomdham, C. 3276 (BKF). Yala: Betong, 27 Aug 1923, Kerr, A.F.G. $7687(\mathrm{~K})$.

LAOS: Champassak: Thorel, C. s.n. (P); ibidem, Thorel, C. s.n. (P).

VIETNAM: Luang, Aug 1868, Pierre, L. 4542 (P). Dong Nai: Bien Hoa, Cochinchine, Pierre, J.B.L. 4541 (P); Gia Lai: Dak Doa, Poilane, E. 18108 (P).

MALAYSIA: Sabah: 24 Feb 1985, Lamb, A.L. 8/85 (E); Interior Zone, Batu Punggol, Apr 1987, Vermeulen, J.J. 1188 (L); Kinabatangan District, Kori Timber Camp, 7 Nov 1948, Cuadra, A. A.2168 (A, K, KEP, SAN, SING); Kinabatangan District, Northern Tabin Wildlife Reserve (Near Tabin River), 16 Oct 2000, Poulsen, A.D. et al. 1659 (E); Lahad Datu, Bukit Baturong, 7 Jul 2000, Kiew, R. RK5040 (SING); Lahad Datu, Tabin Wildlife Reserve, 24 Oct 2000, Kiew, $R$. RK5120 (SING); ibidem, 25 Oct 2000, Pius, G. et al. SAN143649 (E, K); Madai Baturong Forest Reserve, Baturong Hill, 12 Jun 1996, Lim, S.P. et al. LSP738 (SING); Sandakan, Gomantong, 13 Feb 1960, Meijer, W. SAN20756 (A, K, L); Sandakan, Gomantong, Gomantong Caves, 31 Oct 1968, Kokawa, S. \& Hotta, M. 551 (KYO); ibidem, 31 Oct 1968, Kokawa, S. \& Hotta, 
M. 557 (KYO); ibidem, 31 Oct 1968, Kokawa, S. \& Hotta, M. 565 (KYO); ibidem, 25 Sep 1968-26 Sep 1968, Ogata, K. 10975 (KYO, L); Sandakan, Gomantong, Gomantong Hill, 20 Sep 1970, Banggilon, K. SAN66579 (K, L); ibidem, 28 Apr 1996, Lim, S.P. et al. 580 (SING); ibidem, 26 Apr 84, Sands, M.J.S. \& Young, R.G.N. 3936 (E, K); Sandakan, Hutan Simpan Gomanton, Between Gomantong Cave and Bukit Dulong Lambu, 10 Sep 1976, Tamura, M. \& Hotta, M. 602 (KYO); Sandakan, Tabin River, 16 Oct 2000, Vermeulen, J.J. 2037 (SING); Sukau, Panggi, 18 Sep 1996, Kiew, R. \& Lim, S.P. RK4211 (K, SING); Tawao, Oct 1922-Mar 1923, Elmer, A.D.E. 20569 (G, GH, K, SING). Sarawak: 1st Division, 28 May 1975, Burtt, B.L. B.8207 (E); 1st Division, Bau, 24 Apr 1955, Brooke, W.M.A. 9872 (L); 1st Division, Bau District, Burtt, B.L. \& Woods, P.J.B. B.1898 (E); 1st Division, Bau District, Bukit Jebong, 7 Aug 1970, Lehmann, P.F. PFL364 (E); 1st Division, Bau District, Bukit Kapor, 22 May 1962, Burtt, B.L. \& Woods, P.J.B. B.1887 (E); 1st Division, Bau District, Fairy Cave, 9 Feb 1999, Jemree, S. et al. S.82056 (SAR); 1st Division, Bau District, Seburan Mine, 24 May 1962, Burtt, B.L. \& Woods, P.J.B. B.1925 (E); 1st Division, Bau Limestone Hills, Bukit Boring, 14 Nov 1985, Yii et al. S.50353 (K, L); 1st Division, Bau, Gunung Stulang, 16 Oct 2001, Meekiong, K. SBC778 (SING); 1st Division, Bulit Majing, Tebakang, 16 Dec 1988, Kessler 226 (L); 1st Division, Gunung Berloban, 19 Apr 1984, Yii \& Othman S.46225 (K, L); 1st Division, Jambusan, Poak Road, 16 Dec 1989, Frodin, D.G. \& Bin Ismawi, O. 2077 (K); 1st Division, Padawan, Bukit Megetang, 4 Mar 1969, Wright, E. \& Chai, P. S.27457 (E, K); 1st Division, Padawan, Gunung Regu, 6 May 1975, Burtt, B.L. B.8089 (E); 1st Division, Taiton, Yii et al. S.51207 (L, SAR); Bidi, Jul 1893, Ridley, H.N. s.n. (SING); Bidi Cave, Oct 1929, Clemens, J. \& Clemens, M.S. 7434 (NY); ibidem, 22 Oct 1929, Clemens, J. \& Clemens, M.S. 7627 (K); Kuching, Idzumi, H. \& Togashi, M. s.n. (TI); ibidem, 29 Oct 1975-31 Oct 1975, Idzumi, H. \& Togashi, M. s.n. (TI); Kuching to Padawan, 30 Sep 1981, Croat, T.B. 53161 (SAR); Kuching, Daerah Kecil Siburan, Kampong Mambong, 12 Dec 1999, Imaichi, R. et al. 2 (SAR); Kuching, Penrissen, Gunung Braang, 8 Dec 2000, Jemree, S. et al. S.84036 (SAR); Kuching, Penrissen, Mambong, Gunung Bar, 19 Feb 1993, Lai \& Rantai et al. S.66009 (K, SAR); Kuching, Tiang Bekap, Mt. Mentawa, 11 Mar 1967, Chew, W.-L. CWL.1288 (A, AAU, K, L, SING); Kuching, Tiang Bekap, Padawan Road, 3 Apr 1960, Anderson, J.A.R. 12349 (K, L, SING); Samarahan , Serian District, Lobang Mawang, Bukit Selabor, 26 Sep 1968, Ilias bin Paie S.28047 (K, L, SING); ibidem, 26 Sep 1968, Ilias bin Paie S.28051 (K); Tambusan, Sep 1904, Ridley, H.N. s.n. (SING); Mongkos, Gunong Selebur, 20 Apr 1999, Jamree et al. S.82239 (SAR); Serian District, Gunung Niyat, Ulu Sg. Majat, 27 Feb 2002, Jemree, S. \& Enjah, A. S.85584 (SAR). Peninsular Malaysia: Kedah: Baling, 5 Aug 1941, Naven, J.C. 38038 (SING); Kota Setar, G. Keriang, 24 Jul 2009, Rosdi, M. FRI66329 (SING); Pulau Langkawi, Gn. Raya Forest Reserve, 5 Oct 2010, Chan, Y.M. FRI70632 (SING); Pulau Langkawi, Selat Panchor, 23 Nov 1934, Henderson, M.R. 29079 (K, SING); Langkawi, 20 Nov 1941, Corner, E.J.H. s.n. (SING); ibidem, 20 Nov 1941, Corner, E.J.H. s.n. (SING); ibidem, Nov 1889, Curtis, C. 2107 p.p (SING); ibidem, Curtis, C. $2107 a$ (SING); Langkawi, Gunung Raya, 22 Aug 1988, Anthonysamy, S. SA959 (SING); Pulau Langkawi, Pulau Dayang Buting, 23 Aug 1925, Holttum, R.E. 15125 (BO); Langkawi, Pulau Langgun, 4 Nov 1971, Chin, S.C. 1759 (KLU). Kelantan: Bukit Mangga, 20 Feb 2003, Kiew, R. RK5276 (SING); Gua Maka, 19 May 1990, Kiew, R. \& Anthonysamy, S. RK3020 (SING); Kuala Aring, 1 Sep 1992-11 Sep 1992, Hamid H18 (SING). Pahang: Bukit Cheras, 10 Oct 1901, Henderson, M.R. 25205 (NY, SING); ibidem, 26 Nov 1984, Kiew, R. RK1566 (UPM); Gunung Jebak Puyuh, Kiew, R. RK2145 (UPM); Gunung Senyum, 22 Aug 1986, Anthonysamy, S. SA515 (UPM); Kampong Sri Jaya, Bukit Batu, 28 Oct 1986, Kiew, R. RK2369 (SING); Kuala Lipis, 25 Aug 2008, Mohd Hairul, M.A. FRI60060 (SING); Kuantan, Bt Cheras, 25 Aug 1986, Anthonysamy, S. SA558 (UPM); Kuantan, Bukit Panching, 26 Nov 1984, Kiew, R. 
RK1570 (UPM); Maran, Jengka Forest Reserve, Hutan Lipur Jebak Puyuh area, 14 Oct 2008, Mohd Hairul, M.A. FRI60066 (SING); Taman Negara, Batu Kepayang, 3 Oct 1984, Kiew, R. RK1423 (UPM); Taman Negara, Batu Luas, 1 Oct 1984, Kiew, R. RK1349 (UPM); Temerluh, Gn. Jebak Puyuh, Gn. Senyum, 9 Feb 2011, Kamarul Hisham, M. FRI67230 (SING). Perak: Jan 1885, King's Collector 7046 (SING); ibidem, Nov 1880, Kunstler, H. 983 (SING); ibidem, 1890, Ridley, H.N. s.n. (SING); Batu Kurau, Dec 1884, Scortechini 1580 (SING); Chemar, Perak Tong Temple, 23 Oct 1958, Sinclair, J. 9846 (E, L, SING, US); Grik, Hutan Simpan Kemerong limestone area, 18 Nov 2011, Mohd Hairul, M.A. FRI54071 (SING); Ipoh, 1 Dec 1966, Ng, F.S.P. FRI1795 (L, SING); Ipoh, Gunung Rapat, 2 Mar 1959, Allen, B.M. 4272 (SING); ibidem, 14 Jan 1983, Davis 69304 (E); Kampong Jahang, Gunung Kanda, 5 May 1962, Burtt, B.L. \& Woods, P.J.B. B.1820 (E); Larut, Apr 1884, King’s Collector 5872 (SING); Larut \& Matang, Lata Puteh, 22 Nov 2008, Yao, T.L. FRI65393 (SING); Tambun, Rotan Segar, 29 Nov 1960, Allen, B.M. 4654 (SING). Perlis: Besih Hangat, 17 Nov 1929, Henderson, M.R. 22875 (SING); Bukit Bintang Forest Reserve, Bukit Bintang, 9 Aug 1986, Weber, A. 860809-1/1 (E); Mata Ayer F.R., Bukit Rongkit, Kiew, R. RK3712 (KEP); Wang Kelian, 28 Jun 1993, Kiew, R. RK3653 (SING). Selangor: Kuala Lumpur, Jul 1890, Curtis, C. s.n. (SING); Batu Caves, 24 Aug 1908, Ridley, H.N. 13380 (K, SING); ibidem, 4 Sep 1966, Burkill, H.M. HMB4223 (SING); ibidem, 10 Jul 1906, Ernst, A. 1118 (L); ibidem, 23 Jan 1966, Hardial, S. \& Sidek 474 (C, K, L, SING); ibidem, 9 Jun 1999, Kiew, R. RK4711 (SING); ibidem, 15 Sep 1968, Kokawa, S. 6381 (KYO); ibidem, Dec 1898, Ridley, H.N. 8217 (SING); ibidem, Dec 1891, Ridley, H.N. s.n. (SING); ibidem, 30 Oct 1967, Shimizu, T. \& Fukuoka, N. M.14143 (AAU, K, KYO, L, SING); Genting Highlands, Ulu Gombak, 26 Oct 1937, Nur, M. 34253 (SING). Terengganu: Batu Biwa, 22 Oct 1986, Kiew, R. RK2287 (SING).

INDONESIA: Java: s.l., 1857-1861, de Vriese, W.H. s.n. (L); Unknown 2648 (L); Gunung Burung (Gunung Bunder), 19 Dec 1893, Schiffner, V.F. 2567 (A, BO, K, L); Gombak, Sempor, 17 Apr 1936, Brinkman, R. 672 (BO). Jawa Timur: s.l., Coert, J.H. 1093 (L); Kangean Island, Ardjasa, 1920, Backer, C.A.B. 26969 (BO); Kangean Island, Batoe Poetih, 28 Mar 1919, Backer, C.A.B. 27805 (BO); Kangean Island, Tambajangan, 22 Mar 1919, Backer, C.A.B. 27410 (BO); Soekapoera, 3 Jun 1927, Backer, C.A.B. s.n. (BO); Tretes, Gunung Ardjoeno, May 1919, Bremekamp, C.E.B. s.n. (BO); Zuidergebergte, 27 Mar 1927, Backer, C.A.B. \& Posthumus, O. s.n. (BO); Madioen, Ngebel, 16 Mar 1898, Koorders, S.H. 29801 (K, L); Pogal, Mousset 450 (BO, L). Jawa Tengah: Lebak Barang, 12 Jan 1918, Backer, C.A.B. 23287 (BO). Jawa Barat: Pasir Masigit, Feb 1933, Jacobson, E. 213 (BO); Papandajan, Mar 1930, van der Pijl, L. 205 (BO); Bandung, Zollinger, H. 2002 (G, GH, P, S); Bogor, 1909, Backer, C.A.B. s.n. (L); Bogor, Gunung Tjibodas , Feb 1912, Backer, C.A.B. 2480 (L); Cibodas, 1910, Backer, C.A.B. 32476 (BO); ibidem, 1917, Backer, C.A.B. 22091 (BO); ibidem, 1917, Backer, C.A.B. 22091 (BO); ibidem, 18 Mar 1928, van Steenis, C.G.G.J. 616 (BO); Tjiampea, Gunung Tjibodas, 15 Feb 1924, Bakhuizen van den Brink, R.C. 3568 (BO, L); ibidem, 30 Jan 1921, Bakhuizen van den Brink, R.C. 5153 (BO, L); Klappa Noenggal, 1912, Backer, C.A.B. 5848 (BO, L); Padelarang, Kampong Sempang, Coert, J.H. 625 (L); Res. Preanger, Tjidadap, Tjibeber, 23 Feb 1917, Bakhuizen 2608 (L); Tjampea, Burck, W. \& de Monchy, B.J. s.n. (BO); ibidem, 9 Feb 1913, Koorders, S.H. 40438 (BO); ibidem, de Monchy, B.J. s.n. (L); ibidem, 4 May 1895, Hallier, H.G. 91 (L); ibidem, 25 Sep 1904, Hochreutiner, B.P.G. 1916 (G); Ujung Kulon National Park, Tjilintang, 25 Apr 1963, Wirawan, N. 45 (A, BO, K, L, SING); Palimena, Mont. Prope, Junghuhn, F.W. 3 (K, L). Sumatra: s.l., Yates, H.S. 1939 (NY). Aceh: Gajolanden, 25 Feb 1937, van Steenis, C.G.G.J. 9267 (BO, K, L); Gaju and Alas Lands, Pendeng to Bivouac Aer Putih Waterfall, 17 Feb 1937, van Steenis, C.G.G.J. 8879 (BO, L); Gunung Leuser Nature Reserve, Ketambe Research Station, Gunung Giring, 17 Mar 2008, Wilkie, P. et al. PW764 (E); 
Gunung Leuser Nature Reserve, Ketambe, Mt Ketambe, 24 May 1972, de Wilde, W.J.J.O. \& de Wilde-Duyfjes, B.E.E. 12353 (BO, K, L); Ketambe, 17 Mar 2008, Sumadijaya, A. AX360 (BO); Kloet Nature Reserve, South Kloet, Pucuk Lembang, 10 Jul 1985, de Wilde, W.J.J.O. \& de Wilde-Duyfjes, B.E.E. 19894 (BO, L); Pulau Weh, Sabang, 31 Dec 1933, van Steenis, C.G.G.J. 5734 (BO). Lampung: Gunung Rati, 24 Nov 1921, Unknown 208 (BO, L); Muaradua, 13 Apr 1929, de Voogd, C.N.A. 357 (BO, L). Riau: Anambas Islands, Siantan Island, Terempak, 6 Apr 1928, Henderson, M.R. 20277 (K, SING). Sumatera Barat: Jorong Gasang, Lake Maninjau, 18 Jun 2011, Puglisi, C. \& Hughes, M. CP61 (BO); Padang, Indarung, Kampong Putih, 16 Feb 1981, Hotta, M. \& Okada, H. 247 (KYO); Pajakumbuh, Halaban, 2 Feb 1958, Maradji 482 (L, SING). Sumatera Selatan: Aer Telanai, 11 Nov 1929, Van Steenis, C.G.G.J. 3925 (BO). Sumatera Utara: Pulau Tello, Nov 1924, Boden-Kloss, C. s.n. (SING); Batu Islands, Pualu Telo, 23 Jan 1897, Unknown 654 (BO); Upper Langkat, 6 Nov 1938, Lörzing, J.A. 17359 (BO); Wampu, 1918-1919, Lesger 359 (BO). Borneo: Kalimantan Timur: West Koetai, Kombeng, 22 Nov 1925, Endert, F.H. 5143 (BO, L); West Koetai, Lahren, 29 Jun 1925, Endert, F.H. 1762 (BO, L); Tanah Grogot, Batu Kajang, Desa Kasungai, 27 Nov 1979, Ma'roef, A. AM.250 (BO, K); Berau, Sungai Kelai, Gunung Njapa, 18 Oct 1963, Kostermans, A.J.G.H. 21338 (BO, G, K, L); Berau, Tanjung Redeb, Gunung Buntung, 28 Nov 1981-1 Sep 1981, Kato, M. et al. B.11733 (BO, KYO); Kenangan, 12 Aug 1979, Dransfield, J. 4402 (BO). Kalimantan Selatan: 1908, Winkler, H. 2670 (BO, G, K, L); Gunung Serempaka, 26 Nov 1971, Dransfield, J. \& Saerudin, D. 2314 (BO, L); Djaro Dam, 11 Nov 1971, Kuswata 718 (BO); Muara Uja, 21 Nov 1971, Dransfield, J. \& Saerudin, D. 2268 (BO, K, L). Sulawesi Selatan: Bantimurong National Park, 20 Feb 1938, Buwalda, P. 3761 (BO). Sulawesi Tengah: G. Batoe, 1913, Rachmat 439 (BO); Luwuk, 9 Oct 1989, Coode, M.J.E. 5855 (K); Soroako-Wasuponda Road, 7 Jul 1979, van Balgooy, M.M.J. 3922 (L). Sulawesi Tenggarah: Pohara, Muara Sampara, 14 Apr 1929, Kjellberg, G.K. 1299 (BO, S); Pulau Butung, Jismal Camp, 12 Nov 1989, Coode, M.J.E. 6206B (K); Pulau Butung, 17 Feb 1929, Kjellberg, G.K. 195 (S). Sulawesi Utara: Bogani Nani Wartabone National Park, 24 May 2002, Uji, T. 4595 (BO).

Notes. Epithema saxatile is one of the most widely distributed species of Epithema. At first it appears that there are a few different forms of $E$. saxatile, one with petiolate upper leaves, one with sessile upper leaves and one with occasional, solitary upper leaves, but all of these can be found within a single population.

Some collections of this species have strongly variegated leaves but this character does not appear to have any taxonomic significance.

17. Epithema steenisii Hilliard \& B.L.Burtt, Edinburgh J. Bot. 54: 113 (1997). - TYPE: Indonesia, Sumatra, Aceh, Gaju \& Alas Lands, Gadjah to Pendeng, 16 February 1937, van Steenis, C.G.G.J. 8825 (holotype L; isotypes BO (image seen), K, SING). (Fig. 10)

Herb 3-15 cm high, caulescent, indumentum of strigose, setose or hispid hairs; stem 1-2 mm wide with 2-4 nodes, internodes 1-3 cm long. Leaves strongly membranous, petiolate, occasionally with more than one solitary lower leaf; petioles $0.2-4.5 \mathrm{~cm}$ long; blade of lowest leaf 3.2-9 × 1.7-4 cm, upper leaves $1.5-9 \times 0.7-4.7 \mathrm{~cm}$, more or less lanceolate, elliptic, or narrowly ovate, upper portion sometimes curved and upper 
halves asymmetric, if the blade is asymmetrical, one side up to 1.5 times wider than the other, apex acute to more rarely broadly acute, rarely rounded, base sub-truncate, more rarely cuneate to rounded, inserted evenly on petiole or not, margin variable, weakly serrate to bidentate; upper surface densely strigose to villous, hairs $0.75-1$ mm long; lower surface densely pubescent or strigose and setose, hairs to $1.1 \mathrm{~mm}$ long. Inflorescences 2-4 per plant; peduncles $0.5-10.5 \mathrm{~cm}$ long, arising from leaf axils; bracts cucullate, often completely enclosing inflorescence, 9-23 × 5-12 mm, margin dentate, teeth small, lower surface strigose, hairs to $0.6 \mathrm{~mm}$ long, upper surface glabrous or sub-glabrous; pedicels $1.5-3.6 \mathrm{~mm}$ long, often with only strigose hairs to $0.5 \mathrm{~mm}$ long, but sometimes also with additional minute hairs. Calyx $3.5-5 \mathrm{~mm}$ long; tube $2-3 \times 1.2-2.3 \mathrm{~mm}, 1-2$ times as long as wide, lobes $1.5-2.7 \times 0.6-1.5 \mathrm{~mm}$; outside sparsely to densely strigose, hairs straight, occasionally hooked, 0.5-0.8 mm; glabrous inside. Corolla white or pale lilac to lilac, 6.7-9 mm long; tube 4-6.5 × 1-1.5 $\mathrm{mm}$; lobes $2.5 \times 1 \mathrm{~mm}$. Stamen filaments c. $1.2 \mathrm{~mm}$ long; anther characters unknown; staminodes c. $1.2 \mathrm{~mm}$ long. Nectary of two discrete lobes, c. $0.8 \mathrm{~mm}$ high. Ovary sub-cylindrical, c. $0.9 \times 0.8 \mathrm{~mm}$, sub-glabrous to densely pubescent, hairs hooked, $0.05 \mathrm{~mm}$ long and placed on upper portion of ovary; style 3.7-4.6 mm long, glabrous or sub-glabrous, hairs at base of style. Fruit obovate to cylindrical, 1.4-2.3 × 1.7$2.3 \mathrm{~mm}$; operculum $0.5-0.8 \mathrm{~mm}$, sub-glabrous to sparsely pubescent, as ovary. Seed sub-cylindrical or narrowly to broadly ovoid, the ends often only slightly constricted, $0.3-0.6 \times 0.1-0.3 \mathrm{~mm}$, dark brown, pattern straight to spiralled and reasonably regular.

Distribution. Indonesia (Aceh).

Habitat and ecology. Shaded areas, on and among wet (mossy) rocks and in wet sand by streams and rivers at 200-600 m altitude. Flowering and fruiting February, March, May, June, August.

Provisional IUCN conservation assessment. Vulnerable VU D2. This species is only known from the Indonesian province of Aceh in northern Sumatra and many of the populations are from Gunung Leuser National Park. The species is only known to occur below $600 \mathrm{~m}$ altitude and Gunung Leuser has been subjected to continued deforestation despite being a "protected" area (Barber et al., 2002).

Additional specimens examined. INDONESIA: Aceh: Between Lau Simerah and Lau Penanggajan, 20 Mar 1954, Alston, A.H.G. 14535 (A); Gaju \& Alas Lands, Pendeng-Bivouac Aer Putih Waterfall, 17 Feb 1937, van Steenis, C.G.G.J. 8879A (BO, L); Gunung Leuser Nature Reserve, Camp Simpang, 18 Aug 1972, de Wilde, W.J.J.O. \& de Wilde-Duyfjes, B.E.E. 14353 (BO, K, L, US); Gunung Leuser Nature Reserve, Lau Alas Valley, Ketambe, 21 May 1972, de Wilde, W.J.J.O. \& de Wilde-Duyfjes, B.E.E. 12238 (BO, L); Ketambe, Alas River, 9 Jun 1979, de Wilde, W.J.J.O. \& de Wilde-Duyfjes, B.E.E. 18042 (BO, K, L).

Notes. This species is very similar to Epithema tenerum from Sulawesi but can be distinguished by the difference in the indumentum on the outside of the calyx. In 
addition the cucullate bract usually encloses the inflorescence in Epithema steenisii whereas this is variable in E. tenerum. See further discussion in the notes section of Epithema tenerum.

18. Epithema strigosum (C.B.Clarke) Hilliard \& B.L.Burtt, Edinburgh J. Bot. 54: 113 (1997). - Epithema brunonis var. strigosum C.B.Clarke in A.DC. \& C.DC., Monogr. Phan. 5(1): 179. (1883). - TYPE: Indonesia, Sumatra, Gunung Singgalan, Korthals, P.W. 692 (lectotype L, designated by Hilliard \& Burtt (1997) [see also note below]). (Fig. 10)

Herb 6-20 cm high, acaulescent or with first leaves arising from a very short stem; stem 2-4 mm wide with 0-1 nodes, internodes 0-1.5 cm long. Leaves membranous to strongly membranous, petiolate, one lower solitary leaf and one upper, or several leaves in short-stemmed tuft; petioles $0.5-21 \mathrm{~cm}$ long; blade $1-19 \times 0.5-15 \mathrm{~cm}$, cordate to elongate ovate, symmetrical or not, apex acute, base cordate, sub-auriculate or rounded, inserted evenly on petiole or not, margin crenate or dentate, teeth wide at base and mostly with rounded tips; both surfaces with hairs to $0.8 \mathrm{~mm}$ long. Inflorescences 1-4 per plant; peduncles $6.5-23.5 \mathrm{~cm}$ long, originating opposite solitary leaves or at base of petioles; bracts cucullate, completely enclosing the inflorescence, $14-18 \times 8-15$ $\mathrm{mm}$, margin entire to dentate, lower surface setose and strigose, hairs medium density, to $0.4 \mathrm{~mm}$ long, upper surface glabrous to strigose, hairs to medium density, primarily straight or straight and hooked, to $0.4 \mathrm{~mm}$ long; pedicels $1.7-5(-7.5) \mathrm{mm}$ long, finely densely pubescent, hairs hooked, also with or without sparse straight hairs $0.1-0.4 \mathrm{~mm}$ long. Calyx 3.1-5.2 mm long; tube 1.7-4.2 × 1.5-2.7 mm, lobes 1.5-2.3 × 0.8-1.7 $\mathrm{mm}$; sparsely setose and strigose outside, hairs primarily hooked or both straight and hooked, white, to $0.4 \mathrm{~mm}$ long, covering calyx; inside with hairs throughout to only on lobes, hairs straight or both straight and hooked, to $0.13 \mathrm{~mm}$ long. Corolla white, 4.5$8.3 \mathrm{~mm}$ long; tube $3.7-5 \times 1.5 \mathrm{~mm}$; lobes $0.8-3.3 \mathrm{~mm}$ long; margin slightly fimbriate; a partial or complete band of villous hairs in upper half inside. Stamens $2.1-2.4 \mathrm{~mm}$ long; filaments $1.5-1.8 \mathrm{~mm}$ long; anthers c. $0.6 \mathrm{~mm}$ long; staminodes $1.2-1.7 \mathrm{~mm}$ long. Nectary of one or two lobes, $0.5-0.3 \mathrm{~mm}$ long. Ovary $0.8-1 \times 0.7-0.8 \mathrm{~mm}$, densely pilose, hairs straight, white, $0.1-0.3 \mathrm{~mm}$ long, placed on upper portion of ovary; style 3.5-4.6 mm long, glabrous or sparsely pubescent at base of style, hairs straight; stigma c. $0.5 \mathrm{~mm}$ wide. Fruit cylindrical to spherical, 1.5-1.9 × 1.1-2.3 mm; operculum c. 0.6 mm long, pubescence as on ovary. Seed ovoid, sometimes somewhat curved, 0.4-0.5 $\times$ c. $0.1 \mathrm{~mm}$, light to medium brown, pattern almost straight to spiralled, regular.

Distribution. Indonesia (Sumatra).

Habitat and ecology. Lithophytic. Found in shaded, humid areas beside rivers and streams and along paths at 100-1000 m altitude. Flowering and fruiting JanuaryMarch, August. 
Provisional IUCN conservation assessment. Endangered (EN B1ab(iii)). This species has a restricted distribution in West Sumatra in areas subject to continuing degradation of natural habitats.

Additional specimens examined. INDONESIA: Sumatera Barat: Kabupaten Padang Pariaman, Sipisang, 9 Jan 1995, Okada, H. et al. 1343 (BO); Gunung Singgalan, Singalang, Korthals, P.W. s.n. (L); Mt Sago, 9 Mar 1957, Meijer, W. 5637 (L); Padang, Indarung, K. Putih, 16 Feb 1981, Hotta, M., Okada, H. \& Kohyama, T. 319 (KYO); Padang, Indarung, Ladang Padi, K. Putih, 8 Aug 1984, Hotta, M. \& Okada, H. 1087 (KYO); ibidem, 14 Aug 1981, Hotta, M. \& Okada, H. 516 (KYO); Padang, Ladang Padi, 28 Aug 1984, Hotta, M., Okada, H. \& Kohyama, T. 689 (KYO, L); Padang, Ulu Gadut, 10 Jan 1983, Hotta, M., Okada, H. \& Ito, M. 402 (KYO); Padang, Ulu Gadut, Sungai Gadut Gadang, 2 Aug 1984, Hotta, M., Okada, H. \& Kohyama, T. 324 (KYO); ibidem, 2 Aug 1984, Hotta, M., Okada, H. \& Kohyama, T. 339 (KYO).

Notes. Epithema strigosum is most similar to E. philippinum but differs from E. philippinum in the larger calyx, the lack of a minute pubescence on the outside of the calyx and the larger hairs only being sparse, the pubescence inside the calyx is less dense, the leaf margin is crenate rather than dentate or serrate, the corolla can be longer (4.5-8.3 mm long rather than 5-6 mm long) and is reportedly white rather than blue. Epithema strigosum is only found in Sumatra and E. philippinum only in the Philippines. All specimens of Epithema strigosum are from an area of limestone east of Padang.

Korthals s.n. (L0796748) is possibly a duplicate of Korthals 692, the type collection, but has no field number, only a herbarium number of 113 which is also on the lectotype. The plants are very similar and could be the same collection.

19. Epithema tenerum (C.B.Clarke) Hilliard \& B.L.Burtt, Edinburgh J. Bot. 54: 113 (1997). - Epithema brunonis var. tenerum C.B.Clarke in A.DC. \& C.DC., Monogr. Phan. 5(1): 180 (1883). - TYPE: Indonesia, Sulawesi, South Sulawesi, Maros, Zollinger, H. 1172 (lectotype G, designated here; isolectotypes L, P). (Fig. 6, 13)

Herb 5-14(-20) cm high, caulescent; stem 0.8-2 mm wide with 2-3 nodes, internodes $0.5-5(-10) \mathrm{cm}$ long. Leaves thinly membranous to membranous, petiolate, upper leaves opposite or sub-opposite; petiole of lowest leaf $1-5 \mathrm{~cm}$ long, upper petioles $0.2-5 \mathrm{~cm}$ long. Blade of the lowest leaf 2.3-12 × 1.4-8 cm, upper leaves 1.9-10 × 0.9-5 cm, leaves usually broadly lanceolate to narrowly ovate or elliptic, but occasionally broadly ovate or obovate to broadly obovate, symmetrical or not, if asymmetrical one side up to 1.4 times wider than the other, apex acute to broadly acute, base sub-cordate, subtruncate or obtuse, inserted evenly on petiole or not, margin entire to weakly serrate; upper surface primarily strigose, hairs straight, medium density, to $0.7 \mathrm{~mm}$ long; lower surface may dry grey-green, sub-glabrous to pubescent, hairs medium density, to 0.4 $\mathrm{mm}$ long, veins raised with distinct, sparsely to densely pubescent, primarily strigose hairs, to $0.6 \mathrm{~mm}$ long. Inflorescences 1-6 per plant; peduncles $0.3-7(-18) \mathrm{cm}$ long, originating from the terminal and lower axils; bracts small, sub-cucullate or cucullate, 


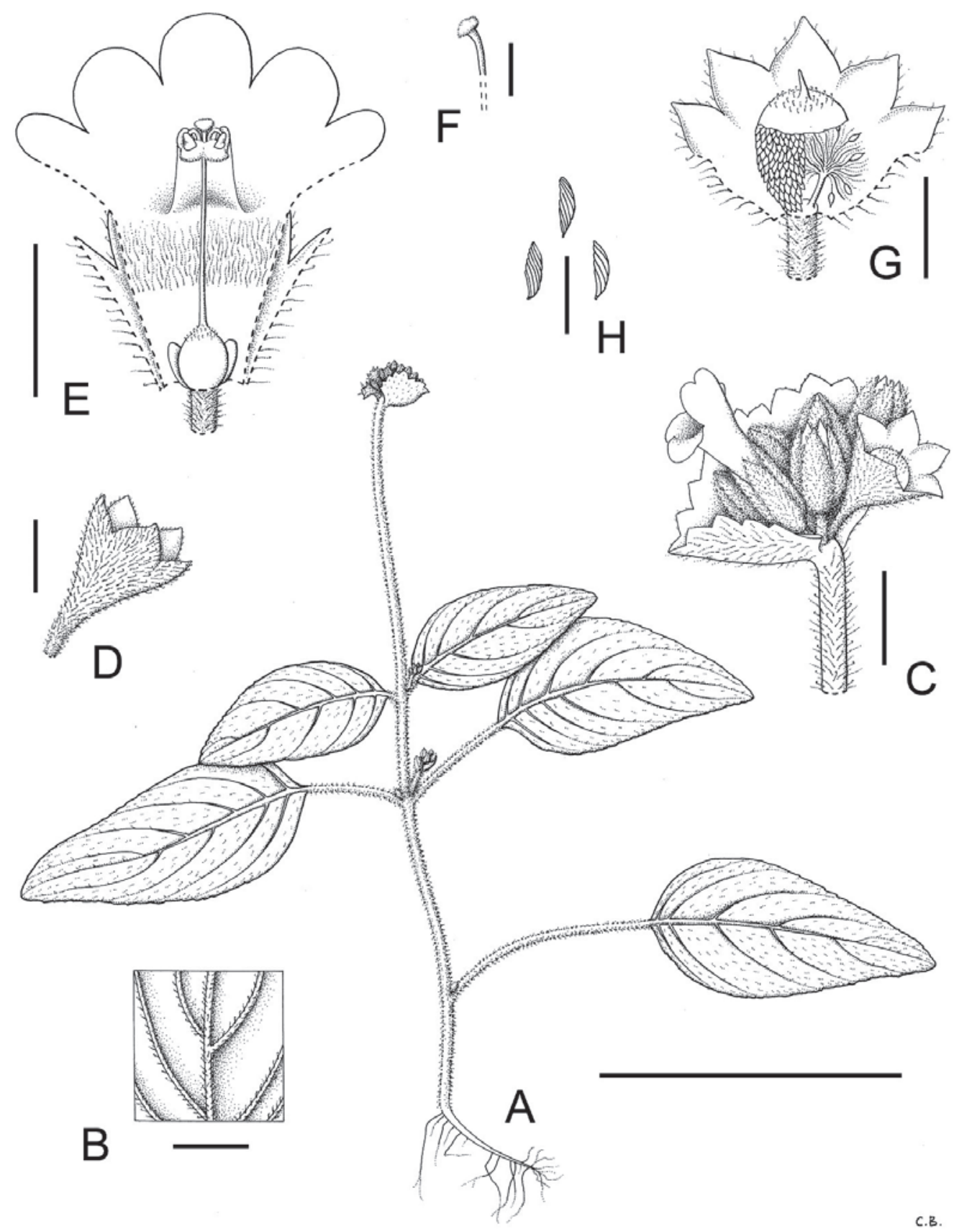

Fig. 13. Epithema tenerum (C.B.Clarke) Hilliard \& B.L.Burtt. A. Habit. B. Underside of leaf. C. Inflorescence. D. Calyx. E. Flower opened out. F. Stigma lateral view. G. Fruit showing seeds, placenta and operculum. H. Seeds. Scale bars: A = $4 \mathrm{~cm} ; \mathrm{B}=5 \mathrm{~mm}$; C, E = $3 \mathrm{~mm}$; D, G $=2 \mathrm{~mm} ; \mathrm{F}=1 \mathrm{~mm} ; \mathrm{H}=0.5 \mathrm{~mm}$. Drawn by Claire Banks from Zollinger $1172(\mathrm{~A})$, Zollinger 1064 (B), P.C. Thomas and W. H. Aroli 09-74 (C-G), Jaag 1600 (H). 
6-16 × 3-7 mm, undulate to lobed with occasional dentation or dentate; lower surface strigose, hairs straight, to medium density and $0.5 \mathrm{~mm}$ long; upper surface glabrous or sub-glabrous; pedicels $0.8-3.3 \mathrm{~mm}$ long, finely pubescent hairs to $0.13 \mathrm{~mm}$ long, also with or without straight, strigose hairs to $0.5 \mathrm{~mm}$ long. Calyx occasionally tinged purple, 1.7-3.4 mm long; tube 0.9-2.5 × 0.9-2.5 mm; lobes 0.6-1.5 × 0.6-1.9 mm; sub-glabrous, strigose or setose outside, also with minute, hooked hairs, hairs medium density, straight hairs to $0.5 \mathrm{~mm}$ long, hooked hairs to $0.3 \mathrm{~mm}$ long; glabrous inside. Corolla blue, pale-blue or pale purple, 5.2-6 mm long, tube 4-4.8 × 0.8-1.3 mm; lobes $0.9-1.8 \times 1-2.1 \mathrm{~mm}$, margins entire to slightly crenate, dense band of villous hair inside. Stamen filaments c. $1.5 \mathrm{~mm}$ long; anther characters unknown; staminodes c.1.25 mm long. Nectary usually of two discrete lobes (occasionally one), margin entire, $1 \times 0.75-1.5 \mathrm{~mm}$. Ovary sub-cylindrical, $0.8-0.9 \times 0.6-0.75 \mathrm{~mm}$, with hooked hair on upper half of ovary, hairs to densely pubescent, c. $0.1 \mathrm{~mm}$ long; style c. 3.3 mm long, glabrous; stigma up to c. 0.5 mm wide. Fruit sub-spherical, 1.6-2.1 × 1.9$2.2 \mathrm{~mm}$, operculum $0.6-0.8 \mathrm{~mm}$ long, indumentum as ovary. Seed narrowly ovoid to ovoid, $0.4-0.5 \times 0.1-0.2 \mathrm{~mm}$, light to medium brown, pattern sub-erect to spiralled, reasonably even.

Distribution. Indonesia (Sulawesi).

Habitat and ecology. Lithophytic, often found directly on limestone or on rocks of unknown type at 20-750 m altitude. Usually beside streams and waterfalls but also on road sides and on rock walls.

Provisional IUCN conservation assessment. Vulnerable (VU D2). This species is only known from a small number of localities and these mostly karst limestone, a habitat which is frequently exploited for cement and tourism.

Additional specimens examined. INDONESIA: Sulawesi Utara: Bolaang Mongondow, Dumoga Bone National Park, Edwards Camp, 26 Mar 1985, de Vogel, E.F. \& Vermeulen, J.J. 6759 (L); Maros, Zollinger, H. 1064 (A, P). Sulawesi Selatan: Bantimurung, 16 Jun 1938, Jaag, O.1600 (L); ibidem, 20 May 1929, Rant, A. 40 (BO); ibidem, 20 Feb 1938, Buwalda, P. 3671 (L); ibidem, 20 Feb 1938, Buwalda, P. 3677 (BO, L); ibidem, 13 Apr 1975, Meijer, W.9133 (BO, L, US); Bantimurung, Bantimurung Waterfall, 15 Apr 2009, Thomas, D. \& Ardi, W.H. 09-74 (E); Pankadjene, 8 May 1931, Teruya, Z. 1848 (SING). Sulawesi Tenggara: Kesali-Porema, 23 Oct 1929, Kjellberg, G.K. 2618 (BO, S).

Notes. This species is most similar to Epithema steenisii from Aceh, Sumatra. It differs mainly in the type of calyx hair, the density of the leaf hair and the length of the corolla. On the calyx there are small, hooked hairs in addition to the longer, straight hairs which are present in both Epithema tenerum and E. steenisii. In addition, the longer hairs are generally shorter in Epithema tenerum than in E. steenisii (to $0.5 \mathrm{~mm}$ vs. to $0.8 \mathrm{~mm}$ long). The hair on the upper surface of the leaves is usually much less dense in Epithema tenerum than in E. steenisii and the corolla tube is $4-4.8 \mathrm{~mm}$ and 4-6.5 mm respectively. 
Epithema tenerum usually follows the general Epithema pattern of having one larger, lower leaf and one or more pairs of opposite, upper leaves. Some upper leaf pairs are actually sub-opposite and this is most clearly seen in the larger specimens (eg. Teruya 1848 (SING)).

The species has been collected from few localities in Sulawesi, principally from Bantimurung in the south-west of the island. The specimens from northern Sulawesi, De Vogel and Vermeulen 6759 (L) and, in particular, Kjellberg 2618 (S), are generally larger than those from Bantimurung. The multi-specimen sheet from Pankadjene, Teruya 1848 (SING), in southern Sulawesi, however, has plants spanning the entire size range.

20. Epithema tenue C.B.Clarke in A.DC. \& C.DC., Monogr. Phan. 5(1): 181 (1883). TYPE: Equatorial Guinea, Bioko, 1863, Mann, G. 2345 (lectotype K, designated here; isolectotype P). (Fig. 14, 15)

Epithema thomense Henriq., Bol. Soc. Brot. 10: 145 (1892). - TYPE: Sao Tome and Principe, Sao Tome, May 1888, Quintas, F. 1272 (lectotype COI, designated here; isolectotype $\mathrm{K})$.

Epithema graniticolum A.Chev., Bull. Soc. Bot. France 58 (Mem. 8d.): 189 (1912). - TYPE: Guinea, Montagne de Boola, Pays de Guerzes, 1048 m, 16 March 1909, Chevalier, A.J.B. 20924 (lectotype P, designated here).

Herb 5-40 cm high, acaulescent or caulescent, occasionally the plant is of only one leaf; stem 1-5 mm wide with 1-3 nodes, internodes 1-16.5 cm long. Leaves membranous or strongly membranous, lower leaves petiolate, upper leaves petiolate and/or sessile or occasionally with a solitary, upper leaf opposite peduncles; petiole of lowest leaf 2-10 cm long, upper petioles $0.2-8 \mathrm{~cm}$ long; blade of lowest leaf 6.5-24 × 4.5-19 $\mathrm{cm}$, upper leaves $2-10 \times 1.5-8.4 \mathrm{~cm}$, the lowest leaf very large and almost oblong to broadly ovate, ovate or cordate, upper leaves ovate to broadly elliptic or spherical, symmetrical or not, apex often rounded but also acute, base cordate or sub-cordate, inserted evenly on petiole or base of upper leaves with one side truncate or obtuse and the other cordate, margin variable, often sub-entire or minutely serrate or dentate, but also serrate, dentate or crenate (usually the upper leaves); upper surface weakly strigose or strigose, hairs to medium density, to $1.5 \mathrm{~mm}$ long; lower surface strigose to medium density, $0.5-0.8 \mathrm{~mm}$ long, and pubescent with minute, hooked hairs to $0.1 \mathrm{~mm}$ long. Inflorescences $1-6$ per plant; peduncles $0.5-14 \mathrm{~cm}$ long, originating from the base of the stem, opposite the solitary lower leaf or from the terminal axil; bracts frequently very large, cucullate and enclosing the inflorescence but may be small and enclosing a small proportion of the inflorescence, 4-40 $\times 5-14 \mathrm{~mm}$, margin dentate or entire; lower surface weakly strigose or strigose, hairs $0.2-0.8 \mathrm{~mm}$ long; upper surface glabrous or sub-glabrous, hairs straight and concentrated towards margins; pedicels $0.8-4 \mathrm{~mm}$ long, weakly strigose, hairs to medium density and 0.8 


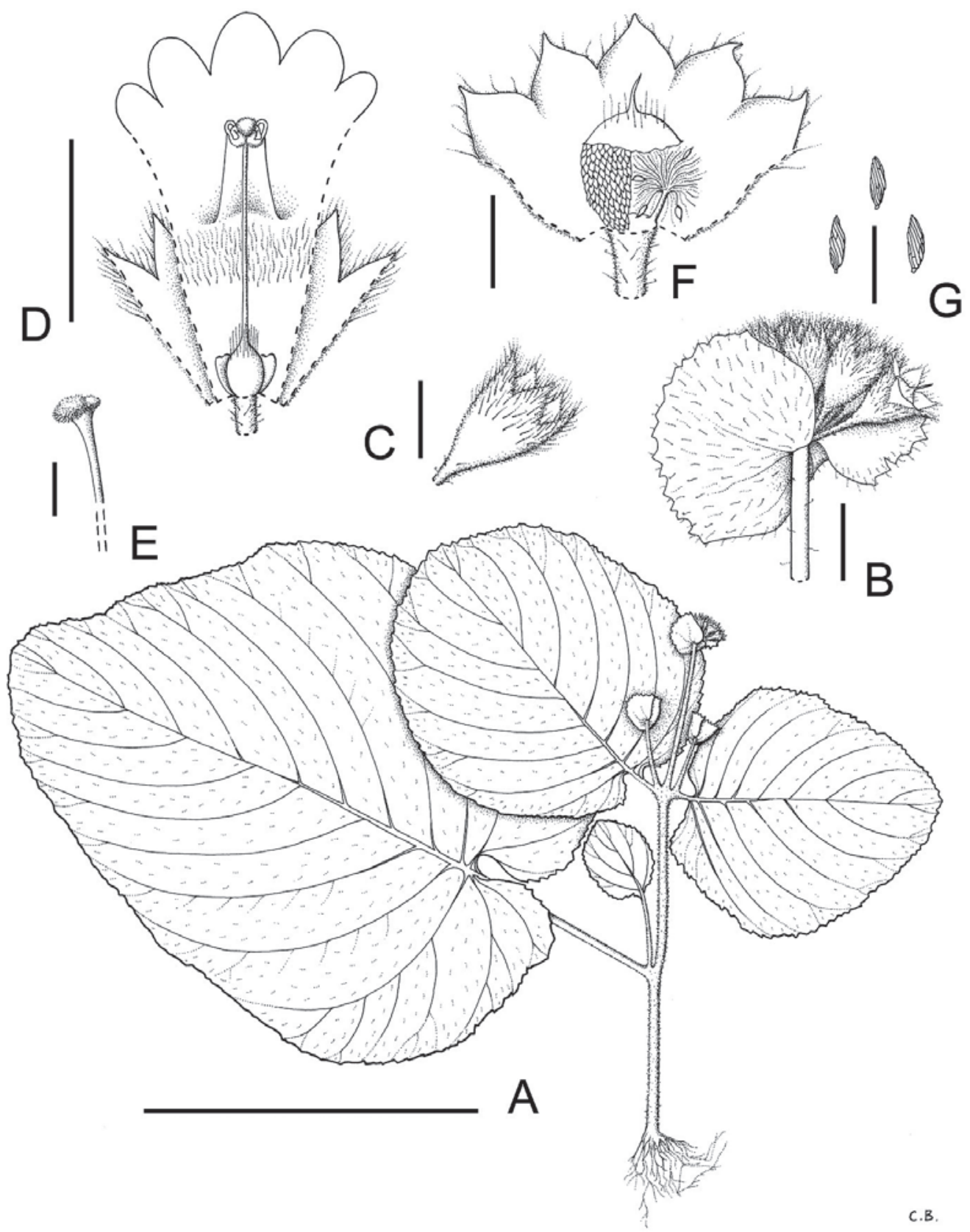

Fig. 14. Epithema tenue C.B.Clarke. A. Habit. B. Inflorescence. C. Calyx. D. Flower opened out. E. Stigma lateral view. F. Fruit showing seeds, placenta and operculum. G. Seeds. Scale bars: $A=10 \mathrm{~cm} ; \mathrm{B}=5 \mathrm{~mm} ; \mathrm{C}, \mathrm{F}=3 \mathrm{~mm} ; \mathrm{D}=6 \mathrm{~mm} ; \mathrm{E}=1 \mathrm{~mm} ; \mathrm{G}=0.5 \mathrm{~mm}$. Drawn by Claire Banks from Letouzy 7722 (A, B), Letouzy 13973 (D, E) and Sita 2886 (C, F, G). 


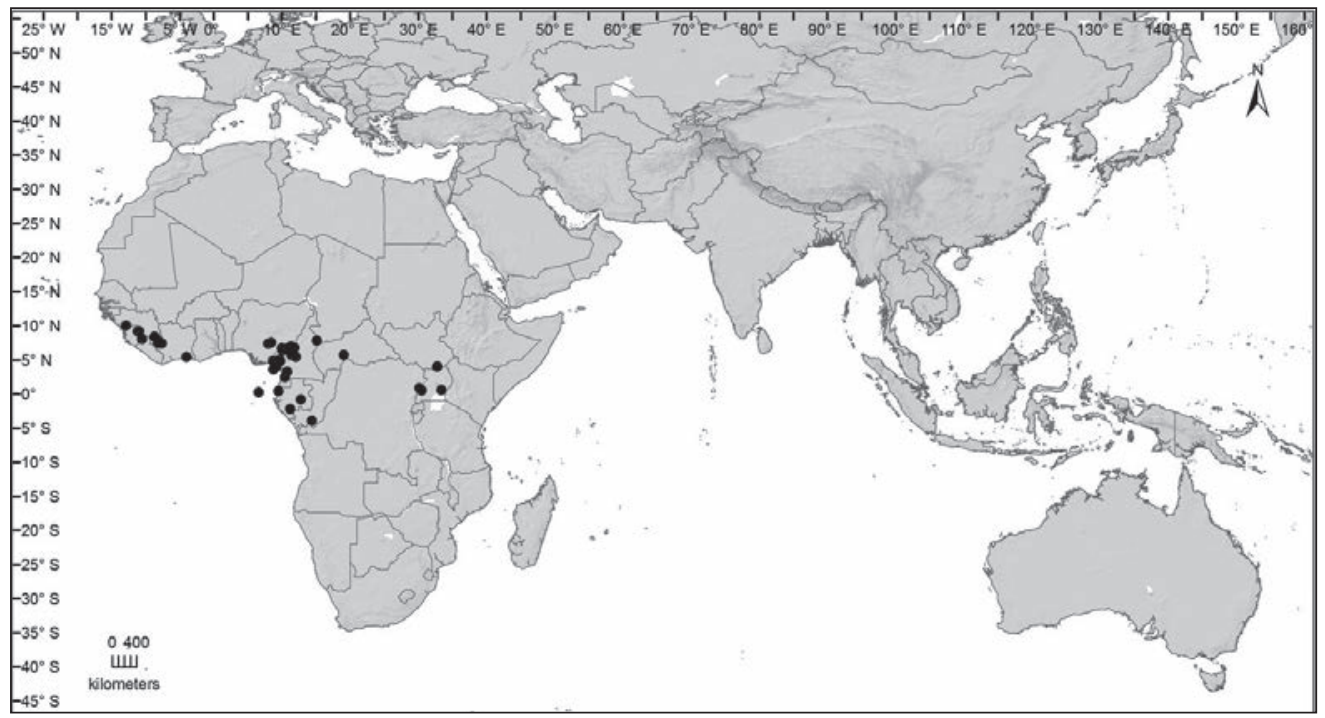

Fig. 15. Distribution of Epithema tenue C.B.Clarke (•).

mm long, also hooked hairs to medium density and $0.1 \mathrm{~mm}$ long. Calyx 2-8 $\times 1.3-2$ $\mathrm{mm}$; tube 1.3-4 mm long; lobes $0.8-3.8 \mathrm{~mm}$ long; with long hairs usually villous, or occasionally to glabrous or sub-glabrous in maturity, hairs to high density, especially in young flowers, hyaline, $0.6-1.5 \mathrm{~mm}$ long; glabrous inside. Corolla blue-purple, tube white, 9.6-10.5 mm long; tube 6.6-7.5 × 2 mm; lobes c. $3 \mathrm{~mm}$ long; a band of villous hairs inside, hairs at half to two-thirds the way up the tube. Stamens 2-2.5 mm long; filaments 1.5-2 mm long; anthers c. $0.5 \mathrm{~mm}$ long; staminodes $1.5-2 \mathrm{~mm}$ long. Nectary usually apparently absent, but also of one or two lobes, sometimes reduced, 0.6-1 $\times$ 0.8-1.3 mm, margin undulate or entire. Ovary ovoid, spherical or cylindrical, 0.8-1.5 $\times 0.6-1.3 \mathrm{~mm}$, weakly hirsute to villous with hairs straight, $0.5-1.5 \mathrm{~mm}$ long, with or without additional hooked hairs to $0.1 \mathrm{~mm}$ long (often seen most easily on the fruit operculum); style $6 \mathrm{~mm}$ long, glabrous or one or two hairs at base, hair to $0.08 \mathrm{~mm}$ long; stigma c. $0.5 \mathrm{~mm}$ wide. Fruit sub-cylindrical to sub-spherical, $1.5-3 \times 1.5-3 \mathrm{~mm}$; operculum $0.5-1 \mathrm{~mm}$ long, indumentum as on ovary. Seed narrowly ovoid to ovoid, 0.4-0.5 × 0.1-0.2 mm, medium brown, pattern sub-erect or erect, reasonably even.

Distribution. Guinea, Sierra Leone, Liberia, Ivory Coast, Nigeria, Cameroon, Equatorial Guinea, Gabon, Sao Tome and Principe, Democratic Republic of Congo, Uganda, South Sudan.

Habitat and ecology. Primarily lithophytic, often on granite at 180-1100 m altitude. Found in humid areas, typically in shade, usually on or near rocky areas, in or beside streams, waterfalls or cave entrances. Also found beside paths and highways. Flowering and fruiting year round. 
Provisional IUCN conservation assessment. Least Concern (LC). This is a widespread species not known to be under any immediate threats.

Additional specimens examined. GUINEA: Kindia, Jacques-Félix, H. s.n. (P).

SIERRA LEONE: Between Kurubonla and Seredu, 15 Nov 1965, Morton, J.K. SL2530 (E, K); Loma Mountains, Oct 1944, Jaeger, P. 227 (K).

LIBERIA: Kolahun, Vahon, 7 Nov 1947, Baldwin, J.T. 10215 (K, US).

IVORY COAST: Chevalier, A.J.B. 21418 (K, P); Chevalier, A.J.B. 21506 (P); Banco, 11 Apr 1979, Knedl, M. 721 (G); Gueoule, 3 Feb 1984, Hepper, F.N. \& Maley, J. 8073 (K); Haut Nuon, Chevalier, A.J.B. 21141 (P); Haut Sassandra, Droupleu to Zoanle, 5 May 1909, Chevalier, A.J.B. 21457 (P); Man, Mt Tankoui, 4 Jul 1966, Ake Assi, L. 9043 (G); ibidem, Aug 1978, Knedl, M. 9/365 (G); Tiapleu, 25 Sep 1955, Unknown 3296 (P).

NIGERIA: Gongola, Mambilla, River Nwum Forest Reserve, 30 Jun 1976, Chapman, J.D. 4505 (K); Sarduna, Gembu, Kamatan Forest Reserve, 14 Dec 68, Daramola, B.O. FHI62313 (K).

CAMEROON: Bamenda Province, Bamenda District, Metschum Falls, 26 Aug 1952, Savory, H.J. UCI299 (K); Bamenda Province, Wum, Nbika’s By-Pass, 28 Jun 1951, Ujor, E. FHI29260 (K); Banyo, Tako-Atta Manga, 5 Jul 1967, Letouzey, R. 8732 (P); Collines a 5km au SW d'Ebianeme-Yong pres Nyabessan, 10 Apr 1968, Letouzey, R. 9238 (P); Ga Village, Pagan Hill, 29 Aug 1966, Letouzey, R. 7722 (COI, K, P); Lolodorf, 15 Jun 1918, Annet, E. 337 (K, P); Muyuka, Munyenge, 29 May 1976, Letouzey, R. 15020 (P); Muyuka, Munyenge, Mt Cameroun, 29 May 1976, Letouzey, R. 15026 (K, P); Nkambe, Vallen Forest, 12 Nov 1974, Letouzey, R. 13198 (P); Northern Cameroon, Munkep, Essu-Munkep Trail, 7 Jul 1975, Letouzey, R. 13973 (P); South West Province, Etinde, Upper Boando, Upper Boando Village, 1 Dec 1993, Cable, S. 283 (K); South West Province, Fako, Buea, Likombe, 2 Sep 1992, Nkeng, P. 90 (K); South West Province, Kupe-Muanenguba Division, Kupe Village, 27 May 1996, Etuge, M. 1973 (K); ibidem, 10 Jul 1996, Kenfack, D. 259 (K); ibidem, 15 Jul 1996, Zapfack, L. 929 (K); South West Province, Kupe-Muanenguba Division, Ngomboku, 10 Dec 1999, Mackinder, B.A. 303 (K); South West Province, Kupe-Muanenguba Division, Nyale, 17 Nov 1988, Cheek, M.R. et al. 9647 (K); South West Province, Kupe-Muanenguba Division, Nyasoso, 24 Jun 1996, Cable, S. 3256 (K, WAG, YA); ibidem, 19 Dec 1993, Cable, S. \& Ajebe, E.F. 644 (K); ibidem, 2 Jun 1996, Etuge, M. 2057 (K); ibidem, 19 Oct 1995, Sidwell, K. et al. 305 (K); South West Province, Kupe-Muanenguba Division, Nyasoso, Mount Kupe, 7 Feb 1996, Cable, S. 3532 (K, YA); ibidem, 7 Oct 1998, Harris, D. 5815 (E); ibidem, 23 Oct 1995, Sebsebe Demissew 4984 (K); ibidem, 7 Jul 1992, Wheatley, J.I. 4117 (K); ibidem, 2 Jun 1996, Zapfack, L. 597 (K); South West Province, Meme, Nyasoso, 23 Jun 1996, Cable, S. 3208 (K); South West Province, Meme, Nyasoso, Mt Kupe, 5 Aug 1993, Balding, S. \& Sivell, D. 37 (K); ibidem, 19 Sep 1992, Cable, S. 19 (K).

EQUATORIAL GUINEA: Bioko, 16 Jan 1947, Guinea, E. 1408 (K); Bioko, Basile, 30 Aug 1990, Carvalho 4464 (K); ibidem, 29 Jul 1986, Carvalho 2130 (K).

GABON: 13 Mar 1925, Le Testu, M.G. 5449 (P); Lastoursville, 1929-1931, Le Testu, M.G. 7018 (K); Libreville, 2 Feb 1899, Klaine, R.P. 1517 (K); ibidem, Klaine, R.P. 2666 (P); Ndende, 22 Nov 1983, de Wilde-Dujfjes, B.E.E. et al. 745 (K).

SAO TOME AND PRINCIPE: St Thome, Henriques, J.A. 5 (K); ibidem, Henriques, J.A. 12 (K).

DEMOCRATIC REPUBLIC OF CONGO: Meya, Camp O.R.S.T.O.M., 12 Feb 1969, Sita, P. 2886 (P). 
UGANDA: 1906, Bagshawe, A.W.G. 1282 (US); Bundibugyo, Sempaya Hot Springs, 14 Sep 1997, Lye, K.A. \& Katende, A.B. LYE22885 (K); Bwamba, Balanga, 22 Nov 1935, Thomas, A.S. Th.1529 (K); Bwamba, Bulanga, 30 Sep 1932, Thomas, A.S. Th.729 (K); Lake Albert Edward, 1906-1907, Bagshawe, A.W.G. 1376 (US).

SOUTH SUDAN: Imatong Mountains, Talanga, 26 Nov 1980, Friis, I. \& Vollesen, K.B. 462 $(\mathrm{C}, \mathrm{K})$.

Notes. There are two forms of Epithema tenue, an acaulescent and a caulescent form. The acaulescent form is found in the Central African Republic, Equatorial Guinea (Bioko), Cameroon and Gabon while the caulescent form is found in the Ivory Coast, Nigeria, Sudan, Uganda, Liberia, Guinea, Sierra Leone, Cameroon and Gabon. In only Cameroon and Gabon are there collections of both forms. The acaulescent form has, primarily, big bracts and inflorescences and calyces which are pubescent with long hairs. The size of the inflorescence is more variable in the caulescent form, but can be still large and pubescent. The duplicates of Harris 5815 (E) from Cameroon show the acaulescent and caulescent forms can be found within one population. In this collection the acaulescent specimens are small and membranous and have small bracts and inflorescences but still have the characteristic long, straight hair on the ovary/ operculum.

Some of the caulescent specimens could be mistaken for Epithema carnosum. They differ from $E$. carnosum by the size of the bract and calyx and the dense indumentum on the outside of the calyx. Some, however, have small bracts, inflorescences and calyces, and the indumentum is sparse. These can still be separated using the length of the hair on the operculum. While this hair is straight in both species, in Epithema tenue it is from $0.5-1.5 \mathrm{~mm}$ long and in E. carnosum it is from $0.08-0.25 \mathrm{~mm}$ long. There are a small number of specimens that are not readily differentiated from Epithema carnosum as the indumentum on the ovary is short. While it is sparse, the indumentum of the calyx of these specimens is that of E. tenue, being hyaline, villous and long instead the hyaline to white, short and more strigose hair of E. carnosum. As these two species occur on different continents confusion between the two should not be a problem.

\section{Excluded Species}

Epithema triandrum (Blanco) Fern.-Vill., Nov App. 150. (1880). - Ophiorrhiza triandra Blanco, Fl. Filip., ed. 2 [F.M. Blanco] 65 (1845). = Ophiorrhiza oblongifolia DC. (see Merrill, 1918).

ACKNOWLEDGEMENTS. We thank the Sibbald Trust for providing the funding for this revision. We thank the directors and curatorial staff of the herbaria that loaned material or hosted visits. Martin Pullan is gratefully acknowledged for his help with the Padme taxonomic database. Claire Banks is thanked for the beautiful illustrations and Serena Lee is thanked 
for her work on the maps. Junji Matsumura (FU) is thanked for providing images of the type of Epithema calcicola and Abdulrokhman Kartonegoro (BO) for images of isotypes of $E$. horsfieldii var. epiphyllum, E. longitubum and E. steenisii. The staff of the Forest Herbarium Bangkok and Pramote Triboun are gratefully acknowledged for enabling and assisting field work in Thailand for the second author. Hannah Atkins and Ruth Kiew are thanked for their thorough reviews of the manuscript.

\section{References}

Barber, C.V., Matthews, E., Brown, D., Brown, T.H., Curran, L. \& Plume, C. (2002). The State of the Forest: Indonesia. Washington DC: World Resources Institute.

Bentham, G. (1835). Scrophularineae Indicae. London: James Ridgway \& Sons.

Blume, C.L. (1826). Bijdragen tot de Flora van Nederlandsch Indie. Batavia: Lands Drukkerij. Burtt, B.L. (1958). Studies in the Gesneriaceae of the Old World. XIII: Miscellaneous transfers and reductions. Notes Roy. Bot. Gard. Edinburgh 22: 305-314.

Burtt, B.L. \& Wiehler, H. (1995). Classification of the family Gesneriaceae. Gesneriana 1: 1-4. Candolle, A.L.L.P. de (1845). Cyrtandraceae. Prodromus 9: 258-286.

Chevalier, A. (1912). Novitates Florae Africanae. Bull. Soc. Bot. France 58 (Mem. 8d.): 189.

Clarke, C.B. (1883). Cyrtandreae. In: Candolle, A.L.L.P. de \& Candolle, A.C.P. de, Monographiae Phanerogamarum 5: 1-303.Decaisne, J. (1834). Description d'un herbier de l'ile de Timor. Nouv. Ann. Mus. Hist. Nat. sér. 3, 3: 333-501.

Don, G. (1838). A General History of the Dichlamydeous Plants, vol. 4 London: Rivington et al.

Henriques, J.A. (1892). Epithema thomense. Bol. Soc. Brot. 10: 145.

Hilliard, O.M. \& Burtt, B.L. (1997). New names in Epithema (Gesneriaceae). Edinburgh J. Bot. 54: 111-113.

IUCN (2001). IUCN Red List Categories and Criteria, Version 3.1. Gland and Cambridge: IUCN Species Survival Commission.

Johns, R.J. (1995). Malesia - An Introduction. Curtis’s Bot. Mag. 12: 52-62.

Li, Z.Y. \& Kao, M.T. (1998). Gesneriaceae. Fl. Taiwan ed. 2, 4: 688-711.

Kiew, R. (1985). Epithema membranaceum, the second Epithema species in Peninsular Malaysia. Malayan Nat. J. 38(3): 36-38.

Mayer , V., Möller, M., Perret, M., \& Weber, A. (2003). Phylogenetic position and generic differentiation of Epithemateae (Gesneriaceae) inferred from cpDNA sequence data. Amer. J. Bot. 90: 321-329.

Merrill, E.D. (1916). Reliquiae Robinsonianae. Philipp. J. Sci., C. 11: 243-319.

Merrill, E.D. (1918). Species Blancoanae. Manila: Bureau of Printing.

Middleton, D.J. (2003). Progress on the Flora of Thailand. Telopea 10: 33-42.

Newman, M., Ketphanh, S., Svengsuksa, B., Thomas, P., Sengdala, K., Lamxay, V. \& Armstrong, K. (2007). A Checklist of the Vascular Plants of Lao PDR. Edinburgh: Royal Botanic Garden Edinburgh.

Ohwi, J. (1943). Epithema. Bot. Mag., Tokyo 57: 125-126.

Spanoghe, J.B. (1841). Prodromus Florae Timorensis. Linnaea 15: 314-350.

Thiers, B. [continuously updated]. Index Herbariorum: A global directory of public herbaria and associated staff. New York Botanical Garden’s Virtual Herbarium. http://sweetgum. nybg.org/ih/ (accessed on 15 Feb. 2015).

Wallich, N. (1832). Plantae Asiaticae Rariores, vol. 3. London: Treuttel \& Würtz. 
Wang, W.T., Pan, K.Y., Li , Z.Y., Weitzman, A.L. \& Skog, L.E. (1998). Gesneriaceae. Flora of China 18: 244-401.

Weber, A. (2004). Gesneriaceae. In: Kubitzki, K. (ed) The Families and Genera of Flowering Plants, vol. 7. Dicotyledons. Lamiales (except Acanthaceae incl. Avicenniaceae). Heidelberg: Springer.

Weber, A., Clark, J.L. \& Möller, M. (2013). A new formal classification of Gesneriaceae. Selbyana 31: 68-94.

Ying, S.S. (1992). Coloured Illustrated Flora of Taiwan, vol. 4. Taipei.

Appendix 1. Index of exsiccatae.

Epithema benthamii C.B.Clarke (1); Epithema carnosum Benth. (2); Epithema ceylanicum Gardner (3); Epithema dolichopodum Hilliard \& B.L.Burtt (4); Epithema horsfieldii (R.Br.) A.DC. (5); Epithema involucratum (Roxb.) B.L.Burtt (6); Epithema longipetiolatum (Merr.) Hilliard \& B.L.Burtt (7); Epithema longitubum Hilliard \& B.L.Burtt (8); Epithema madulidii Hilliard \& B.L.Burtt (9); Epithema membranaceum (King) Kiew (10); Epithema parvibracteatum Hilliard \& B.L.Burtt (11); Epithema philippinum (Hilliard \& B.L.Burtt) Bransgrove (12); Epithema pusillum (C.B.Clarke) Bransgrove (13); Epithema rennellense Hilliard \& B.L.Burtt (14); Epithema sarawakense Hilliard \& B.L.Burtt (15); Epithema saxatile Blume (16); Epithema steenisii Hilliard \& B.L.Burtt (17); Epithema strigosum (C.B.Clarke) Hilliard \& B.L.Burtt (18); Epithema tenerum (C.B.Clarke) Hilliard \& B.L.Burtt (19); Epithema tenue C.B.Clarke in A.DC. \& C.DC. (20).

Aet \& Idjan 32 (7), 942 (7); Ahmad 1 (15); Ake Assi, L. 9043 (20); Allen, B.M. 4272 (16), 4654 (16); Almeida, S.M. s.n. (13); Alston, A.H.G. 14535 (17); Anderson, J.A.R. et al. S.26090 (15); Anderson, J.A.R. 12349 (16), S.20957 (15), S.31770 (15); Anderson, J.A.R. \& Keng, H. K100 (15); Annet, E. 337 (20); Anthonysamy, S. SB7 (4); SA515 (16), SA558 (16); SA959 (16); Argent, G.C.G. et al. 905 b (15), 29038 (1); Argent, G.C.G. C8754a (7); Argent, G.C.G. \& Collins, M. 733 (15); Argent, G.C.G. \& Kerby, R. 621 (15); Ashton, P.S. A.339 (15), A.340 (15); Atha, D. 4785 (2).

Backer, C.A.B. s.n. (16), 2480 (16), 2789 (5), 5848 (16), 6607 (5), 6687 (5), 11469 (5), 22091 (16), 23287 (16), 26969 (16), 27410 (16), 27805 (16), 32476 (16), 36621 (5); Backer, C.A.B. \& Posthumus, O. s.n. (16); Bagshawe, A.W.G. 1282 (20), 1376 (20); Bakhuizen van den Brink, R.C. 2608 (16), 3568 (16), 5153 (16); Balakrishnan, N.P. 10815 (2); Balding, S. \& Sivell, D. 37 (20); Baldwin, J.T. 10215 (20); Banggilon, K. SAN66579 (16); Barnes, E. 928 (3), 1056 (3), 1300 (3); Bartlett, H.H. 7740 (10), 14487 (3), 14744 (3), 15380 (3); Beaman, J.H. 10068 (4), 11727 (15); Beumée, J.G.B. 1328 (5); Bin Rajab, M.K. 714 (16); Bloembergen, S. 3458 (8), 3496 (8); Blume, C.L. s.n. (16), 1029 (16); Boden-Kloss, C. s.n. (16); Boerlage, J.G. 528 (7); Brahmam, M. 2776 (3); Brandis, D. 676 (3); Bremekamp, C.E.B. s.n. (16); Brinkman, R. 672 (16); Brooke, W.M.A. 9872 (16); Brown, R. s.n. (6); Bünnemeijer, H.A.B. 10713 (5), 10890 (5); Burck, W. \& de Monchy, B.J. s.n. (16); Burkill, H.M. HMB4223 (16); Burtt, B.L. B.8089 (16), B.8207 (16), B.8227 (15), B.8336 (15); Burtt, B.L. \& Woods, P.J.B. B.1820 (16), B.1887 (16), B.1898 (16), B.1925 (16), B.2003 (15), B.2230 (15); Buwalda, P. 3671 (19), 3677 (19), 3761 (16), 5110 (7), 5202 (7), 5748 (7), 6006 (7), 7225 (5), 7542 (5).

Cable, S. 19 (20), 283 (20), 3208 (20), 3256 (20), 3532 (20); Cable, S. \& Ajebe, E.F. 644 (20); Carvalho 2130 (20), 4464 (20); Chai, P. S.30372 (15); Chan, Y.M. FRI70632 (16); Chantharanothai, P. et al. 90/303 (16); Chapman, J.D. 4505 (20); Charoenphol, C. et 
al. 3661 (16), 3662 (16), 3976 (10); Cheek, M.R. et al. 9647 (20); Chermsirivathana, C. 1 (3), 413 (16); Chevalier, A.J.B. 20924 (20), 21141 (20), 21418 (20), 21457 (20), 21506 (20); Chew, W.-L. CWL.293 (15), CWL.1017 (15), CWL.1288 (16); Chin, S.C. 392 (11), 1404 (10), 1514 (10), 1759 (16); Ching, R.C. 6421 (2); Clarke, C.B. 8997 (2), 12300 (2), 15665 (2), 19076 (2), 24805 (2), 36192 (2); Clason, E.W. C99 (5); Clemens, J. \& Clemens, M.S. 7434 (16), 7627 (16); Clemens, M.S. 724 (1); Coert, J.H. 625 (16), 1093 (16); Coode, M.J.E. 5313 (1), 5855 (16), 6206B (16); Corner, E.J.H. s.n. (16); Coster s.n. (5); CP, BS \& BN 2997 (16); Croat, T.B. 53161 (16); Cuadra, A. A.2168 (16); Cuming, H. 823 (3), 1265 (1); Curtis, C. s.n. (16), 955 (10), 2107 (16), 2107a (16).

Daramola, B.O. FHI62313 (20); Davis 69304 (16); de Monchy, B.J. s.n. (16); de Vogel, E.F. \& Vermeulen, J.J. 6759 (19); de Voogd, C.N.A. 357 (16); de Vriese, W.H. s.n. (16); de Wilde, J.J.F.E. 1155 (1); de Wilde, W.J.J.O. \& de Wilde-Duyfjes, B.E.E. 12238 (17), 12353 (16), 14353 (17), 18042 (17), 19894 (16), 21918 (5), 21940 (5); de Wilde-Dujfjes, B.E.E. et al. 745 (20); Deschamps s.n. (3); Dhal, N.K. \& Rout, N.C. 8122 (3); Dissing, H. 2738 (14), 2798 (14); Dransfield, J. 3869 (1), 4402 (16); Dransfield, J. \& Saerudin, D. 2268 (16), 2314 (16); Duthie, J.F. 2990 (2).

Eberhardt 3363 (3); Edaño, G. s.n. (1), 418 (1), 435 (1), $435 a$ (1), 720 (1), 746 (1), 758 (1), 1965 (1), 3070A (1), 3478 (1), BS41748 (1), BS77711 (4), BS77570 (4); Ellis, J.L. 20452 (3); Elmer, A.D.E. 8318 (1), 9698 (1), 13470 (1), 15626 (1), 15877 (1), 17551 (1), 18283 (1), 20569 (16); Endert, F.H. 1762 (16), 5143 (16); Ernst, A. 1118 (16); Escritor, L. BS21452 (1); Esquirol, J. 711 (2); Etuge, M. 1973 (20), 2057 (20); Eyma, P.J. 1826 (7), 2574 (7).

Faden, R.B. \& Faden, A.J. 76/494 (3); Fenix, E. BS26049 (3), BS28106 (3); Feuilletau de Bruyn, W.K.H. 335 (1); Forrest, G. 15699 (2); Fosberg, F.R. 57278 (3); Frodin, D.G. \& Bin Ismawi, O. 2077 (16).

Gachalian, F. 147 (1); Gafui, I.H. \& collectors BSIP14759 (14), BSIP14763 (14); Gallatly, G. 1035 (16); Gardner, G. 606 (3); Geesink, R. \& Phengklai, C. 6082 (16), 6231 (16); Geoffray, M. 95 (3); Grey-Wilson, C. \& Grey-Wilson, C.M. 3016 (3); Grupe, D.A. 200 (3); Guinea, E. 1408 (20).

Haegens, R.M.A.P. \& Klazenga, N. 515 (15); Hallier, H.G. 91 (16), 4128 (1); Hamid H18 (16); Hansen, C. 17 (15); Hardial, S. \& Sidek 474 (16); Harris, D. 5815 (20); Haviland, G.D. s.n. (15); Haviland, G.D. \& Hose, C. 3525 R (15); Henderson, M.R. 19406 (10), 20277 (16), 22382 (10), 22580 (10), 22875 (16), 25010 (10), 25205 (16), 29079 (16); Hennipman, E. 610 g (7); Henriques, J.A. 5 (20), 12 (20); Henry, A. 1916 (3), 12280 (2), 12705 (2), 13497 (2); Hepper, F.N. \& Maley, J. 8073 (20); Hicks, D. 151 (1); Hochreutiner, B.P.G. 1916 (16); Holttum, R.E. 15125 (16); Hooker, J.D. \& Thomson, T. s.n. (2), 2161 (2); Hoover, W.S. et al. HW11417 (5), Deden 151 (5); Horsfield, T. Cyrtandra 3 (5), 85 (5); Hotta, M. et al. 313 (18), 319 (18), 324 (18), 339 (18), 402 (18), 689 (18); Hotta, M. 14402 (15), 15281 (15); Hotta, M. \& Okada, H. 247 (16), 516 (18), 1087 (18); Hsieh, C.F. et al. s.n. (3).

Iboet 221 (6); Idjan 175 (1); Idzumi, H. \& Togashi, M. s.n. (16); Ilias bin Paie S.28047 (16), S.28051 (16); Imaichi, R. et al. 2 (16), 30 (15), 38 (15); Imin, K. FRI63208 (11).

Jaag, O. 1600 (19); Jacques-Félix, H. s.n. (20); Jacobson, E. 199 (5), 213 (16); Jaeger, P. 227 (20); Jaheri 1522 (15); Jamree et al. S.82239 (16); Jayasuriya, A.H.M. 843 (3); Jemree, S. \& Enjah, A. S.85584 (16); Jemree, S. et al. S.82056 (16), S.84036 (16); Jeswiet, J. 670 (5); Julaihi S.86868 (15); Julaihi et al. S.76938 (15); Julia, S. et al. S.93376 (15), S.94757 (15), S.99303 (15); Junghuhn, F.W. 3 (16). 
Kamarul Hisham, M. FRI67230 (16); Kanehira, R. \& Hatusima, S. 11848 (1), 13143 (1); Kasem 641 (3); Kato, M. et al. C.5728 (7), C.5232 (7), C.5144 (7), C.6007 (7), C.6908 (1), C.11441 (7), B.11733 (16); Keenan, J. et al. 798 (16); Kenfack, D. 259 (20); Kerr, A.F.G. 2655 (16), 6381 (16), 7687 (16), 12385 (16), 13053 (3), 13183 (16), 15775 (10), 15893 (10), 19385 (16); Kessler 226 (16); Kiew, R. et al. RK4321 (4); Kiew, R. RK525 (15), RK1231 (10), RK1347 (10), RK1349 (16), RK1423 (16), RK1498 (11), RK1566 (16), RK1570 (16), RK1584 (10), RK1590A (10), RK2145 (16), RK2287 (16), RK2369 (16), RK3653 (16), RK3712 (16), RK4711 (16), RK4759 (4), RK5040 (16), RK5120 (16), RK5254 (10), RK5276 (16); Kiew, R. \& Anthonysamy, S. RK3020 (16); Kiew, R. \& Lim, S.P. RK4138 (4), RK4211 (16); King, G. s.n. (2); King's Collector 5872 (16), 7046 (16); Kjellberg, G.K. 195 (16), 314 (6), 315 (6), 1299 (16), 2616 (1), 2618 (19); Klaine, R.P. 1517 (20), 2666 (20); Knedl, M. 9/365 (20), 721 (20); Kochummen, K.M. FRI26221 (11); Kokawa, S. 6381 (16); Kokawa, S. \& Hotta, M. 551 (16), 557 (16), 565 (16), 1073 (4), 1125 (4); Koorders, S.H. 29801 (16), 40438 (16); Kooy, C.W. 403 (5), 789 (5); Kornassi 482 (1), 621 (7), 967 (7); Korthals, P.W. s.n. (18), 692 (18); Koster, C. BW10773 (1), BW13665 (1); Kostermans, A.J.G.H. 1103 (16), 1346 (16), 1401 (16), 18053B (5), 18629 (5), 18839 (5), 21338 (16); Kostermans, A.J.G.H. \& Soegeng 914 (1); Kulkarni, B.G. 108673 (13); Kunstler, H. 983 (16); Kuswata 105 (5), 121 (5), 213 (5), 718 (16).

Lace, J.H. 4892 (2); Lai \& Rantai et al. S.66009 (16); Lamb, A.L. $8 / 85$ (16); Larsen, K. et al. 722 (16), 2923 (3), 3363 (16), 30967 (16), 41243 (16), 41413 (16), 41450 (16), 41713 (10), 42245 (10), 42312 (10), 42471 (10), 42579 (10), 42584 (10), 43589 (3), 43771 (3), 44271 (10), 46055 (16), 46161 (2), 46426 (3), 46861 (3); Le Testu, M.G. 5449 (20), 7018 (20); Lehmann, P.F. S.30091 (15), PFL364 (16), PFL596/S. 30372 (15), S.30091 (15); Leong, P. et al. PL215 (15), PL320 (15); Lesger 359 (16); Letouzey, R. 7722 (20), 8732 (20), 9238 (20), 13198 (20), 13973 (20), 15020 (20), 15026 (20); Lim, S.P. et al. 580 (16), LSP675 (4), LSP681 (4), LSP738 (16); Lobb, T. 387 (3); Loeters, J.J. 1625 (5); Loher, A. 1554 (3), 1555 (3), 1556 (3), 4231 (1), 6668 (3), 6669 (3); Lopez, G. BS42031 (1), BS42049 (1), BS41343 (9); Lörzing, J.A. 17359 (16); Lucas 1025 (3); Lütjeharms, W.J. 4598 (15), 5112 (15); Lye, K.A. \& Katende, A.B. LYE22885 (20).

Mackinder, B.A. 303 (20); Madulid, D.A. et al. 11563 (9); Maier Sarip, R. 162 (5); Mann, G. 2345 (20); Maradji 482 (16); Marcan, A. 1869 (3); Ma'roef, A. AM.250 (16); Martin, P.J. S.39273 (15); Maxwell, J.F. s.n. (16), 72-362 (16), 73-107 (3), 73-285 (16), 74-814 (16), 75-958 (16), 84-55 (16), 86-723 (16), 86-893 (10), 87-1154 (3), 89-920 (2), 93-886 (16), 95-536 (3), 99-278 (2); McGregor, R.C. BS19951 (1), BS43566 (3); McKee, H.S. 1958 (3), 6317 (3); Meekiong, K. SBC778 (16); Meijer, W. 5637 (18), 9133 (19), 10045 (1), SAN20756 (16); Mendoza, D.R. \& Convocar, P P. 226 (12), 297 (12); Mendum, M. et al. 34 (5), 43 (1), 113 (1), 180 (1); Merrill, E.D. 52 (3), 4216 (3), 5106 (1), 8222 (1); Middleton, D.J. et al. 4055 (16), 4284 (3), 4330 (16), 4345 (16), 4428 (16), 4544 (3), 4579 (3), 5350 (16), 5368 (16), 5425 (16), 5524 (16), 5613 (3), 5687 (3), 5768 (3); Middleton, D.J. \& Triboun, P. 4846 (3); Mohd Hairul, M.A. FRI54071 (16), FRI60060 (16), FRI60066 (16); Mohidin S.21608 (15); Moktar, A. et al. S.49467 (15); Mooney, H.F. 2067 (3), 2754 (3), 3853 (2), 3891 (2), 4045 (2), 4092 (2); Morton, J.K. SL2530 (20); Mousset 450 (16); Mrs. General Walker s.n. (3), 140 (3); Murata, G. et al. T.15146 p.p. (3), T.15146 p.p. (2), T.15156 (2), T.16922 (3), T.17092 (16), T.38467 (16), 38544 (16), T.38587 (16).

Nadiah, I. et al. SAN149136 (4); Nair, K.K.N. 1354 (3); Namba, T. \& Mikage, M. 82-413 
(3); Nanakorn, W. 4240 (3); Nanakorn, W. et al. 4664 (2); Narayanaswami, M.A. 478 (2); Naven, J.C. 38038 (16); Ng, F.S.P. FRI1795 (16); Nielsen, I. 17 (15), 416 (15); Nimanong, B. \& Phusomsaeng, S. 288 (3); Niyomdham, C. 3276 (16); Nkeng, P. 90 (20); Nooteboom, H.P. 5217 (7), 5357 (1); Norsaengsri, M. et al. 7965 (3), 8048 (3); Norsaengsri, M. 10568 (3); Nur, M. 3585 (16), 34253 (16).

Officers of H.M.S. "Penguin” s.n. (14); Ogata, K. 10975 (16); Okada, H. et al. 1343 (18); Okada, H. \& Tsukaya, H. OT-48 (7).

Palee, P. 73 (3), 544 (3); Parish, C.S.P. 63 (3), 133 (3); Parnell, J. et al. 95-335 (16); Petrmitr, O. 70 (3); Phengklai, C. 2933 (16); Phuong, V.X. 311 (2); Phusomsaeng, S. \& Bunchuai, K. 42 (3); Phusomsaeng, S. et al. 19 (10), 83 (16); Pierre, J.B.L. 4541 (16), 4542 (16); Pius, G. et al. SAN143649 (16); Pleyte, D.R. 150 (1), 1053 (1); Poilane, E. 18108 (16); Pooma, R. et al. 2100 (3), 2697 (3), 4298 (10), 4580 (2), 4824 (3), 6486 (10), 6578 (10), 6579 (10); Pooma, R. 1059 (3); Poulsen, A.D. et al. 1659 (16); Pradeep, A.K. 5850 (3); Prayad 1034 (3), 1422 (3); Prieditis, N. s.n. (15); Primack, R.B. S.42402 (15); Puglisi, C. \& Hughes, M. CP61 (16); Put 219 (3), 410 (2), 1015 (3), 1051 (16), 1853 (3), 4118 (10).

Quintas, F. 1272 (20); Quisumbing, E. BS78105 (3).

Rabil 318 (16); Rachmat 439 (16); Raghavan, R.S. 90185 (3); Ramamoorthy, T.P. HFP2007 (3), HFP2035 (3); Ramos, M. BS1435 (3), BS13624 (3), BS32892 (3), BS42176(1); BS43313 (1), BS76791 (1); Ramos, M. \& Edaño, G. BS29466 (3), BS48897 (1), BS49227 (1); Reynoso \& Majaducon PPI27265 (1); Ridley, H.N. s.n. (16), 8217 (16), 11883 (11), 13380 (16); Riedel, Mr. s.n. (1); Robinson, C.B. 1727 (7), BS9525 (3), BS9534 (3), BS17216 (1); Rogstad, S.H. 728 (15); Rosdi, M. FRI66329 (16).

Sabli, J. et al. S.89048 (15); Sakol 1150 (3); Saldanha, C.J. 14426 (3); Sands, M.J.S. 6141 (1), 6318 (1), 6337 (1), 6841 (1); Sands, M.J.S. \& Young, R.G.N. SANDS3936 (16); Sangkhachand, B. 1405 (10); Santisuk, T. \& B.N. 451 (10); Savory, H.J. UCI299 (20); Saw, L.G. FRI39945 (10); Schiffner, V.F. 2567 (16); Schmutz, E. 2127 (8), 3208 (8), 3623 (5), 4027 (5), 4345 (5), 4732 (5); Schram, F.A.W. BW10631 (1), BW13259 (1); Scortechini 1580 (16); Scott 3 (2); Sebsebe Demissew 4984 (20); Sharma, B.D. 42032 (3); Shimizu, T. et al. T.18519 (3), T.19413 (3), T.20909 (2), T.27111 (16), T.27551 (16), T.27571 (16), T.28985 (16), T.27111 (16), T.27100 (16), T.27103 (16), T.27551 (16), T.28519 (3); Shimizu, T. et al. T.10789 (3), T.29151 (16); Shimizu, T. \& Fukuoka, N. M.14143 (16); Shimizu, T. \& Stone, B.C. 13739 (11), 14427 (10); Sidwell, K. et al. 305 (20); Sinclair, J. 7821 (11), 9846 (16); Sirute'e, B. et al. BSIP9621 (14); Sita, P. 2886 (20); Smith, H.C. 16273 (2); Smitinand, T. 3489 (16), 4719 (3); Smitinand, T. \& Abbe, E.C. 6149 (16); Smitinand, T. \& Sleumer, H.O. 1006 (3), 1109 (3), 1207 (16); Sorensen, T. et al. 4235 (3), 4936 (3); Spanoghe, J.B. 226 (6); Staples, G.W. et al. 1409 (3); Stocks s.n. (13); Stocks, Law \& co. s.n. (3); Stone, B.C. et al. 870424-5/1 (10); Stone, B.C. 8830 (11), 13730 (15); Stone, B.C. \& Anderson, E.F. SAN86719 (4); Stone, B.C. \& Mahmud 8400 (11); Subba Rao, G.V. 28210 (2); Subramanyam, K. 8644 (3), 8975 (3); Sugau, J. JBS96 (15); Sukendar SK740 (1); Sulit, M.D. \& Conklin, H.C. 16913 (3); Sumadijaya, A. AX360 (16); Sumathi, R. 17895 (3); Sumithraarachchi, D.B. DBS388 (3); Sutheeson, S. 2489 (16), 5004 (16); Suvanakoses, P. 1727 (10), 2060 (3); Symington, C.F. \& Agama, J. 9361 (4); Synge, P.M. S.634 (15), S.565 (15).

Tagawa, M. et al. T.11259 (16); Tamura, M. \& Hotta, M. 602 (16), 690 (4); Teijsmann, J.E. s.n. (7); Teruya, Z. 1848 (19); Theobald, W.L. \& Grupe, D.A. 2368 (3), 2404 (3); Thomas, A.S. Th.729 (20), Th.1529 (20); Thomas, D. \& Ardi, W.H. 09-74 (19); Thomson, G. 
s.n. (3), 121 (3); Thomson, T. s.n. (2); Thorel, C. s.n. (16); Thwaites, G.H.K. 2844 (3); Toxopeus, F.J. 358 (1); Turner, I.M. \& Yong 158 (10).

Uji, T. 4595 (16); Ujor, E. FHI29260 (20); Unknown s.n. (3), 147 (11), 208 (16), 226 (6), 654 (16), 753 (3), 2648 (16), 3296 (20), 3847 (2), 12200 (2); Unknown (ex Herb. Lugd. Batav.a) s.n. (5).

Vajvavelu, E. 27558 (3); van Balgooy, M.M.J. 3600 (1), 3922 (16), 4712 (1), 4889 (7), 5066 (7); van Borssum Waalkes, J. 3025 (6); van der Pijl, L. 205 (16); van Niel, J.P. 3554 (15); van Pruk, L. 350 (3); van Royen, P. 4946 (1), 5063 (1); van Steenis, C.G.G.J. 616 (16), 3925 (16), 5734 (16), 7788 (5), 8825 (17), 8879 p.p. (16), $8879 A$ (17), 9267 (16), 11079 (5); Verheijen, J.A.J. 3070 (8), 3323 (8); Vermeulen, J.J. 1188 (16), 2037 (16); Versteegh, C. BW7405 (1), BW12694 (1); Vink, W. BW12141 (7).

W. C. 2680 (3); Waas, S. 1407 (3); Wallich, N. 9080 (10); Walsh, M.E. 62 (8); Weber, A. 860809 $1 / 1$ (16); Weber, C.M. 1440 (1); Wenzel, C.A. 231 (1), 437 (1); Wheatley, J.I. 4117 (20); Wight, R. 1/162 (3), 556 (3), 2350 (3); Wilkie, P. et al. PW764 (16); Wilkie, P. FRI52899 (10); Williams, K. et al. 1400 (16), 2033 (16); Williams, R.S. 937 (3), 2046 (1), 2401 (1); Winkler, H. 2670 (16); Wirawan, N. 45 (16); Wolff, T. 3004 (14), 3025 (14); Wongprasert, T. 9912-30 (16); Wright, E. \& Chai, P. S.27457 (16).

Yang, K.C. 3465 (3); Yao, T.L. FRI65393 (16); Yates, H.S. 1939 (16); Yii \& Othman S.46225 (16); Yii et al. S.50353 (16), S.51207 (16); Ying, S.S. s.n. (3).

Zapfack, L. 597 (20), 929 (20); Zollinger, H. 1064 (19), 1172 (19), 2002 (5), 2612 (5). 
